\title{
CLASSIFICATION OF FINITE $p$-GROUPS WITH CYCLIC INTERSECTION OF ANY TWO DISTINCT CONJUGATE SUBGROUPS
}

\author{
ZVONIMIR JANKO \\ University of Heidelberg, Germany
}

\begin{abstract}
We give a complete classification of non-Dedekindian finite $p$-groups in which any two distinct conjugate subgroups have cyclic intersection (Theorems A, B and C).
\end{abstract}

\section{INTRODUCTION}

The purpose of this paper is to give a complete classification of finite nonDedekindian $p$-groups (i.e., $p$-groups that possess non-normal subgroups) in which any two distinct conjugate subgroups have cyclic intersection (Problem 1572 stated in [3]).

In Theorem 16.2 in [1], Theorem A and Theorem B are completely determined finite non-Dedekindian $p$-groups all of whose non-normal subgroups are either cyclic, abelian of type $(p, p)$ or ordinary quaternion. Since in these groups any two distinct conjugate subgroups have a cyclic intersection, so these results can be considered as a good start in solving problem 1572. Therefore, after proving Theorems $\mathrm{A}$ and $\mathrm{B}$, we may always assume that there is in a title group $G$ a non-normal subgroup which is neither cyclic nor abelian of type $(p, p)$ nor an ordinary quaternion group and such groups will be completely determined in Theorem C. Now we state our main results.

TheOREM A. Let $G$ be a p-group all of whose non-normal subgroups are cyclic or abelian of type $(p, p)$. Assume in addition that $G$ possesses a nonnormal abelian subgroup of type $(p, p)$. Then $G$ is one of the following groups

2010 Mathematics Subject Classification. 20D15.

Key words and phrases. Finite p-groups, 2-groups of maximal class, Dedekindian $p$ groups, ordinary quaternion group, maximal non-normal subgroups, conjugate subgroups. 
(where $\mathrm{S}\left(p^{3}\right), p>2$, denotes the nonabelian group of order $p^{3}$ and exponent p):

(a) $G \cong \mathrm{D}_{16}$ or $\mathrm{SD}_{16}$.

(b) $G=L Z$, where $L \cong \mathrm{S}\left(p^{3}\right), p>2$, is normal in $G, Z \cong \mathrm{C}_{p^{2}}, L \cap Z=$ $\mathrm{Z}(L)=\mathrm{Z}(G)$.

(c) $G$ is any nonabelian group of order $p^{4}$ with an elementary abelian subgroup of index $p$.

(d) $p=2$ and $G \cong\left(\mathrm{D}_{8} * \mathrm{Q}_{8}\right) \times \mathrm{C}_{2}$, where $\mathrm{D}_{8} \cap \mathrm{Q}_{8}=\left(\mathrm{D}_{8}\right)^{\prime}$ or $G \cong$ $\mathrm{H}_{16} * \mathrm{Q}_{8}$ with $\mathrm{H}_{16} \cap \mathrm{Q}_{8}=\left(\mathrm{H}_{16}\right)^{\prime}$, where $\mathrm{H}_{16}$ is the nonmetacyclic minimal nonabelian group of order 16 .

(e) $G \cong \mathrm{M}_{p^{s+1}} \times \mathrm{C}_{p}, s \geq 3$.

(f) $G=(Z * S) \times \mathrm{C}_{p}$, where $Z \cong \mathrm{C}_{p^{s+1}}, s \geq 1, Z \cap S=S^{\prime}$, and either $p=2$ and $S \cong \mathrm{D}_{8}$ or $p>2$ and $S \cong \mathrm{S}\left(p^{3}\right)$ or

$G=Z * S$, where $Z \cong \mathrm{C}_{p^{s+1}}, s \geq 1, Z \cap S=S^{\prime}$, and $S$ is the nonmetacyclic minimal nonabelian group of order $p^{4}$.

(g) $G$ is an $\mathrm{A}_{2}$-group of order $p^{5}$ from Proposition 71.4(b2) in [2] for $\alpha=1$.

(h) $G \cong \mathrm{Q}_{8} * \mathrm{Q}_{8} * \mathrm{Q}_{8}$, an extraspecial group of order $2^{7}$ and type " - ".

(i) $G=\left(A_{1} * A_{2}\right) \mathrm{Z}(G)$, where $A_{1}$ and $A_{2}$ are minimal nonabelian $p$-groups and $\mathrm{Z}(G)$ is cyclic. In case $p=2, A_{1}$ and $A_{2}$ are isomorphic to one of $\mathrm{D}_{8}, \mathrm{Q}_{8}$ and $\mathrm{M}_{2^{n}}, n \geq 4$, where in case $A_{1} \cong \mathrm{Q}_{8}$ and $A_{2} \cong \mathrm{D}_{8}$ we must have $|\mathrm{Z}(G)|>2$. In case $p>2, A_{1}$ and $A_{2}$ are isomorphic to one of $\mathrm{S}\left(p^{3}\right)$ or $\mathrm{M}_{p^{n}}, n \geq 3$.

Conversely, all the above groups satisfy the assumptions of the theorem.

TheOREM B. Let $G$ be a 2-group all of whose non-normal subgroups are either cyclic, abelian of type $(2,2)$ or ordinary quaternion. Assume in addition that $G$ possesses a non-normal subgroup $H$ which is isomorphic to $\mathrm{Q}_{8}$. Then $G$ is isomorphic to one of the following groups :

(a) $G \cong \mathrm{Q}_{32}$ (a generalized quaternion group of order 32 ).

(b) $G$ is a unique 2-group of order $>2^{4}$ with the property that $\Omega_{2}(G) \cong$ $\mathrm{Q}_{8} \times \mathrm{C}_{2}$ and we have $|G|=2^{5}$, where this group (of class 3 ) is defined in part A2(a) of Theorem 49.1 in [2].

(c) $G$ is a splitting extension of a cyclic noncentral normal subgroup of order 4 by $\mathrm{Q}_{8}$.

(d) $G=H_{1} \times H_{2}$, where $H_{1} \cong H_{2} \cong \mathrm{Q}_{8}$.

(e) $G=\left\langle h_{0}, h_{1}\right\rangle\langle g\rangle$, where $\left\langle h_{0}, h_{1}\right\rangle \cong \mathrm{Q}_{8}, \mathrm{Z}\left(\left\langle h_{0}, h_{1}\right\rangle\right)=\langle z\rangle,\langle g\rangle \cong \mathrm{C}_{2^{n}}$, $n \geq 3,\left\langle h_{0}, h_{1}\right\rangle \cap\langle g\rangle=\{1\}, \Omega_{1}(\langle g\rangle)=\left\langle z^{\prime}\right\rangle, g^{2} \in \mathrm{Z}(G),\left[g, h_{0}\right]=1$, and $\left[g, h_{1}\right]=z^{\epsilon} z^{\prime}, \epsilon=0,1$. Here we have $|G|=2^{n+3}, n \geq 3, G^{\prime}=\Omega_{1}(G)=$ $\left\langle z, z^{\prime}\right\rangle \cong \mathrm{E}_{4}, G$ is of class 2 and $\mathrm{Z}(G)=\left\langle g^{2}\right\rangle \times\langle z\rangle \cong \mathrm{C}_{2^{n-1}} \times \mathrm{C}_{2}$.

(f) $G=C * Q$, where $C \cong \mathcal{H}_{2}=\left\langle a, b \mid a^{4}=b^{4}=1, a^{b}=a^{-1}\right\rangle, Q \cong \mathrm{Q}_{8}$ and $C \cap Q=\left\langle a^{2} b^{2}\right\rangle=Q^{\prime}$.

Conversely, all the above groups satisfy the assumptions of the theorem. 
TheOrem C. Let $G$ be a p-group with a cyclic intersection of any two distinct conjugate subgroups. Assume in addition that $G$ has a non-normal subgroup which is neither cyclic nor abelian of type $(p, p)$ nor an ordinary quaternion group. Then $G$ is metabelian and $G$ is either a 2-group of maximal class and order $\geq 2^{5}$ (if $|G|=2^{5}$, then $G \cong \mathrm{D}_{32}$ or $\mathrm{SD}_{32}$ ) or $G$ is a p-group of class at most 3 with $G^{\prime} \neq\{1\}$ elementary abelian of order at most $p^{2}$ and $G$ is isomorphic to one of the groups defined in Propositions 3(b2), 5, 7, 8, 9, 10, 11 and 12 stated in the section 4.Proof of theorem $C$.

Conversely, all these groups satisfy the assumptions of our theorem.

In this paper we shall consider only finite $p$-groups and our notation is standard (see [1]).

\section{Proof of Theorem A}

Let $G$ be a $p$-group all of whose non-normal subgroups are cyclic or abelian of type $(p, p)$ and we assume that $G$ possesses a non-normal abelian subgroup $H$ of type $(p, p)$. We set $K=\mathrm{N}_{G}(H)$ so that we have $H<K<G$ and $K \unlhd G$. Since each subgroup $X$ of $G$ with $X>H$ is normal in $G$, it follows that $K / H$ is Dedekindian and $K / H$ has exactly one subgroup of order $p$. This implies that $K / H \neq\{1\}$ is either cyclic or $p=2$ and $K / H \cong \mathrm{Q}_{8}$. Let $L / H$ be a unique subgroup of order $p$ in $K / H$ so that $L \unlhd G$ and $\Omega_{1}(K) \leq L$. If $g \in G-K$, then $L=\left\langle H, H^{g}\right\rangle$ and so we have $\Omega_{1}(K)=L$.

Suppose that $K$ does not possess a $G$-invariant abelian subgroup of type $(p, p)$. By Lemma 1.4 in [1], we get $p=2$ and $K$ is of maximal class. But $H$ is a normal four-subgroup in $K$ and so $K \cong \mathrm{D}_{8}$. Since $\mathrm{C}_{G}(H)=\mathrm{C}_{K}(H)=H$, it follows by a result of M. Suzuki (see Proposition 1.8 in [1]) that $G$ is also a 2-group of maximal class. In this case $H$ has exactly two conjugates in $K=L \cong \mathrm{D}_{8}$ and so $|G: K|=2$ and $|G|=2^{4}$. It follows that $G \cong \mathrm{D}_{16}$ or $\mathrm{SD}_{16}$ and we have obtained the groups stated in part (a) of our theorem.

In what follows we may assume that $K$ possesses a $G$-invariant abelian subgroup $U$ of type $(p, p)$. Since $\Omega_{1}(K)=L$, we have $U \leq L$ and so $L=H U$ with $|H \cap U|=p$. If $L$ is abelian, then $L \cong \mathrm{E}_{p^{3}}$. If $L$ is nonabelian, then in case $p>2$ we have $L \cong \mathrm{S}\left(p^{3}\right)$ and in case $p=2$ we must have $L \cong \mathrm{D}_{8}$. But the last case cannot happen since $U \unlhd G$ and $L$ has exactly two four-subgroups which would imply that also $H \unlhd G$, a contradiction. Hence we have either $L \cong \mathrm{E}_{p^{3}}$ or $p>2$ and $L \cong \mathrm{S}\left(p^{3}\right)$.

Suppose that $p>2$ and $L \cong \mathrm{S}\left(p^{3}\right)$. In that case we have

$$
\langle z\rangle=H \cap U=L^{\prime}=\mathrm{Z}(L) \leq \mathrm{Z}(G) .
$$

If $\mathrm{C}_{G}(L)>\langle z\rangle$, then take an element $x \in \mathrm{C}_{G}(L)-\langle z\rangle$ such that $x^{p} \in\langle z\rangle$ and consider the abelian subgroup $S=\langle h, z, x\rangle$ of order $p^{3}$, where $h$ is any element in $H-\langle z\rangle$. By our assumptions, we have $S \unlhd G$. But $L \cap S=H=\langle h, z\rangle$ and so $H \unlhd G$, a contradiction. We have proved that $\mathrm{C}_{G}(L)=\langle z\rangle$. Since an 
$\mathrm{S}_{p}$-subgroup of $\operatorname{Aut}(L)$ is isomorphic to $\mathrm{S}\left(p^{3}\right)$, it follows that $|G: L|=p$ and $K=L$ so that $|G|=p^{4}$. Also note that $G /\langle z\rangle \cong \mathrm{S}\left(p^{3}\right)$ and $G / K$ acting on $p+1$ subgroups of order $p^{2}$ (containing $\langle z\rangle$ ) fixes $U$ and acts transitively on $p$ other ones. Hence $U$ is the unique $G$-invariant subgroup of order $p^{2}$ in $L$. Set $V=\mathrm{C}_{G}(U)$ so that $V$ is an abelian normal subgroup of order $p^{3}$ in $G$ and we have $G=L V$ with $L \cap V=U$. If $V \cong \mathrm{E}_{p^{3}}$, then we get a group stated in part (c) of our theorem. Hence we may assume that there is an element $t$ of order $p^{2}$ in $V-U$ such that $t^{p}=z$. We have obtained a group from part (b) of our theorem.

From now on we may assume that $L \cong \mathrm{E}_{p^{3}}$. If $|G / L|=p$, then $K=L$ is elementary abelian of order $p^{3}$ and index $p$ and again we have obtained the groups from part (c) of our theorem. Thus we may assume in what follows that $|G / L|>p$.

In the rest of the proof we fix our notation for:

$$
\mathrm{E}_{p^{2}} \cong H, K=\mathrm{N}_{G}(H) \neq G, \Omega_{1}(K)=L, \mathrm{E}_{p^{2}} \cong U \unlhd G,
$$

where

$$
L=H U, H \cap U \cong \mathrm{C}_{p},
$$

and $\{1\} \neq K / H$ is either cyclic or $p=2$ and $K / H \cong Q_{8}$. Also we fix our assumptions that $L \cong \mathrm{E}_{p^{3}}$ and $|G / L|>p$.

(i) First assume that there is a central element $z$ in $G$ of order $p$ which is contained in $H$.

In that case we have $|G: K|=p$ so that $K>L$ and therefore there is an element $v \in K-L$ of order $p^{2}$ with $v^{p} \in L-H$. We may choose a $G$-invariant subgroup $U \leq L$ of order $p^{2}$ so that $U \leq \mathrm{Z}(G)$. The socle $\Omega_{1}(X)$ of any cyclic subgroup $X$ in $G$ of composite order is contained in $U$.

Indeed, acting with $G / K$ on $p+1$ subgroups of order $p^{2}$ in $L$ which contain $\langle z\rangle$, we see that $|G: K|=p$. Since $|G / L|>p$, we have $K>L$ and so there is an element $v \in K-L$ of order $p^{2}$, where $v^{p} \in L-H$. Considering $\langle v, z\rangle \cong \mathrm{C}_{p^{2}} \times \mathrm{C}_{p}$, we obtain

$$
\langle v, z\rangle \unlhd G \text { and so } \mho_{1}(\langle v, z\rangle)=\left\langle v^{p}\right\rangle \unlhd G .
$$

Then we may set $\mathrm{E}_{p^{2}} \cong U=\left\langle z, v^{p}\right\rangle \leq \mathrm{Z}(G)$. Let $X$ be any cyclic subgroup of composite order in $G$ and assume that $\Omega_{1}(X) \not Z U$. But then $\Omega_{1}(X) \leq K$ and so $\Omega_{1}(X) \leq L$. Take an element $1 \neq u \in U \leq \mathrm{Z}(G)$ and consider the subgroup $X \times\langle u\rangle \unlhd G$ so that we get $\Omega_{1}(X) \unlhd G$. Since $\Omega_{1}(X) \not \leq U$, we get $L \leq \mathrm{Z}(G)$ and so $H \unlhd G$, a contradiction.

(i1) Suppose that $K / L$ is noncyclic. Then we have $p=2, K / H \cong \mathrm{Q}_{8}$, $|G|=2^{6}$ and $K / L \cong \mathrm{E}_{4}$. Since $\mho_{1}(K) \leq U \leq \mathrm{Z}(G), K / U$ is elementary abelian. Considering the Dedekindian group $G / U$ of order $2^{4}$ which possesses an elementary abelian subgroup $K / U$ of index 2 , it follows that $G / U$ is abelian and so $G^{\prime} \leq U$. Any two non-commuting elements in $G$ generate here a 
minimal nonabelian subgroup (see Lemma 65.2 in [2]). For any $g, h \in G$ we have $\left[g^{2}, h\right]=[g, h]^{2}=1$ and so $\mho_{1}(G) \leq \mathrm{Z}(G)$. In particular, for any $g \in G-K, g^{2} \in K-L$ is not possible and so $g^{2} \in L$ and this implies $g^{2} \in U$. Hence $\mho_{1}(G) \leq U$ and $\exp (G)=4$. Since $\mathrm{Z}(G) \leq K$, we get $\mathrm{Z}(G)=U$. Because $G / L \cong \mathrm{E}_{8}$, we have $\mathrm{C}_{G}(L)>L$ and so $\mathrm{C}_{G}(L) \leq K$ implies $\mathrm{C}_{K}(L)>L$. Thus there is $v \in \mathrm{C}_{K}(L)-L$ such that $v^{2} \in U-H$. Let $h \in H-U$ and consider the subgroup $\langle h, v\rangle \cong \mathrm{C}_{2} \times \mathrm{C}_{4}$ so that $\langle h, v\rangle \unlhd G$ and

$$
\Omega_{1}(\langle h, v\rangle)=\left\langle h, v^{2}\right\rangle \unlhd G .
$$

If $\left\langle h, v^{2}\right\rangle \not Z \mathrm{Z}(K)$, then there is $g \in G-K$ centralizing $\left\langle h, v^{2}\right\rangle$, a contradiction. We have proved that $H \leq \mathrm{Z}(K)$ and so $\mathrm{C}_{G}(L)=K$.

We have $\mathrm{Z}(K)=L$ and so $\left|K^{\prime}\right|=2$ and $U=K^{\prime} \times(H \cap U)$. Suppose that $\mho_{1}(K)=U$. Then there are elements $v_{1}, v_{2} \in K-L$ such that $z_{1}=v_{1}^{2} \neq$ $z_{2}=v_{2}^{2}$, where $z_{1}, z_{2} \in U-H$. Let $h \in H-U$ and $g \in G-K$. Since

$$
\left\langle h, v_{1}\right\rangle \cong \mathrm{C}_{2} \times \mathrm{C}_{4} \text { and }\left\langle h, v_{2}\right\rangle \cong \mathrm{C}_{2} \times \mathrm{C}_{4},
$$

we have

$$
\left\langle h, v_{1}\right\rangle \unlhd G \text { and }\left\langle h, v_{2}\right\rangle \unlhd G \text { and so }\left\langle h, z_{1}\right\rangle \unlhd G \text { and }\left\langle h, z_{2}\right\rangle \unlhd G .
$$

But this gives $h^{g}=h z_{1}=h z_{2}$ and $z_{1}=z_{2}$, a contradiction.

We have proved that $\mho_{1}(K)=\langle u\rangle$ is of order 2 , where $u \in U-H$. It follows that $K /\langle u\rangle$ is elementary abelian and so $\mho_{1}(K)=K^{\prime}=\langle u\rangle$. Let $k_{1}, k_{2} \in K-L$ be such that $\left\langle k_{1}, k_{2}\right\rangle$ covers $K / L$. Since $k_{1}^{2}=k_{2}^{2}=u$ and $\left[k_{1}, k_{2}\right]=u$, we get $Q=\left\langle k_{1}, k_{2}\right\rangle \cong \mathrm{Q}_{8}$ and $K=H \times Q, L=H \times\langle u\rangle$, where $Q \unlhd G$.

Since $G^{\prime} \leq U$ is elementary abelian, it follows that $G$ induces on $Q$ only inner automorphisms of $Q$ and so we have $G=Q * C$, where $C=\mathrm{C}_{G}(Q)$ and $Q \cap C=\langle u\rangle, K \cap C=L$. Also we have $\mathrm{Z}(C)=\mathrm{Z}(G)=U$. By Lemma 1.1 in [1] we get $\left|C^{\prime}\right|=2$. On the other hand, let $h \in H-U, g \in C-L$ and $v \in Q$ with $v^{2}=u$. Since

$$
\mathrm{C}_{2} \times \mathrm{C}_{4} \cong\langle h, v\rangle \unlhd G \text {, it follows that } \Omega_{1}(\langle h, v\rangle)=\langle h, u\rangle \unlhd G .
$$

Thus we get $h^{g}=h u$ and so $u \in C^{\prime}$. We have proved that $C^{\prime}=Q^{\prime}=\langle u\rangle=G^{\prime}$.

Let $g$ be an element in $C-L$ and $h \in H-U$. If $g^{2} \in U-\langle u\rangle$, then $C=\langle g, h\rangle \cong \mathrm{H}_{16}$, where $\mathrm{H}_{16}$ denotes the nonmetacyclic minimal nonabelian group of order 16 . If $g^{2} \in\langle u\rangle$, then we have $\langle g, h\rangle \cong \mathrm{D}_{8}$ and so in this case $C=\langle g, h\rangle \times\langle z\rangle$, where $\langle z\rangle=H \cap U$. We have obtained the groups stated in part (d) of our theorem.

(i2) Suppose that $\{1\} \neq K / L$ is cyclic so that $K / H$ is cyclic of order $\geq p^{2}$. In this case we show that $G / L$ is abelian.

Indeed, assume that $G / L$ is nonabelian. Since $G / L$ is Dedekindian, it follows that $p=2$ and $G / L \cong \mathrm{Q}_{8}$. We also have $\Omega_{1}(G)=L$. Since $\mathrm{C}_{G}(L)>L$ 
and $\mathrm{C}_{G}(L) \leq K$, we get $\mathrm{C}_{K}(L)>L$. Let $v \in \mathrm{C}_{K}(L)-L$ with $o(v)=4$ so that $v^{2} \in U-H$ and let $h \in H-U$. Then

$$
\mathrm{C}_{2} \times \mathrm{C}_{4} \cong\langle h, v\rangle \unlhd G, \text { and }\left\langle h, v^{2}\right\rangle \unlhd G .
$$

If $\left\langle h, v^{2}\right\rangle \not \leq \mathrm{Z}(K)$, then there is $g \in G-K$ which centralizes $h$, a contradiction. Hence $\left\langle h, v^{2}\right\rangle \leq \mathrm{Z}(K)$ and so $H \leq \mathrm{Z}(K)$ which implies that $K$ is abelian.

Since $G / U$ is Dedekindian and nonabelian, it follows that $G / U$ is Hamiltonian. Let $Q / U$ be a subgroup in $G / U$ which is isomorphic to $\mathrm{Q}_{8}$ and set

$$
Q_{0} / U=\mathrm{Z}(Q / U)=(Q / U)^{\prime} .
$$

Let $Q_{1} / U$ and $Q_{2} / U$ be two distinct cyclic subgroups of order 4 in $Q / U$ so that $Q_{1}$ and $Q_{2}$ are abelian and $Q_{1} \cap Q_{2}=Q_{0}$. It follows that $Q_{0} \leq \mathrm{Z}(Q)$ and so $Q_{0}=\mathrm{Z}(Q)$. By Lemma 1.1 in [1], $\left|Q^{\prime}\right|=2$ and since $Q^{\prime}$ covers $Q_{0} / U$, it follows that $Q_{0}=U \times Q^{\prime} \cong \mathrm{E}_{8}$. But then $Q_{0}=\Omega_{1}(G)=L$ and so $K=\mathrm{C}_{G}(L) \geq Q$ is nonabelian, a contradiction. We have proved that $G / L$ is abelian and so $G / L$ is either cyclic of order $\geq p^{2}$ or $G / L$ is abelian of type $\left(p^{s}, p\right), s \geq 1$.

(i2a) Assume that $G / L$ is cyclic. Let $g \in G-K$ so that $\langle g\rangle$ covers $G / L$ and let $\langle t\rangle=\Omega_{1}(\langle g\rangle)$ be the socle of $\langle g\rangle$, where $t \in U-H$ and $o(g)=p^{s}$, $s \geq 3$. We may set $t=g^{p^{s-1}}$ and so $\left\langle g^{p}\right\rangle$ covers $K / H \cong \mathrm{C}_{p^{s-1}}$. Also set $v=g^{p^{s-2}}$ so that $\langle v\rangle \cong \mathrm{C}_{p^{2}}$ and $v^{p}=t$.

Since $\langle g\rangle$ stabilizes the chain $L>U>\{1\}$, it follows that $\left\langle g^{p}\right\rangle$ centralizes $L$ and so $K$ is abelian. Consider the abelian subgroup $\langle h, v\rangle \cong \mathrm{C}_{p} \times \mathrm{C}_{p^{2}}$, where $h$ is any element in $H-U$. Since $\langle h, v\rangle \unlhd G$, we get

$$
\Omega_{1}(\langle h, v\rangle)=\langle h, t\rangle \unlhd G .
$$

Thus we get $h^{g}=h t^{i}$ for some $i \not \equiv 0(\bmod p)$ and so $G^{\prime} \geq\langle t\rangle$. On the other hand,

$$
\mathrm{Z}(G)=\mathrm{C}_{K}(g)=\left\langle g^{p}, U\right\rangle \text { and so }|G: \mathrm{Z}(G)|=p^{2} .
$$

By Lemma 1.1 in [1], we get

$$
|G|=p|\mathrm{Z}(G)|\left|G^{\prime}\right| \text { and so }\left|G^{\prime}\right|=p \text { and } G^{\prime}=\langle t\rangle \text {. }
$$

We have $\langle g, h\rangle \cong \mathrm{M}_{p^{s+1}}$ and if we set $\langle z\rangle=H \cap U$, then

$$
G=\langle z\rangle \times\langle g, h\rangle \cong \mathrm{C}_{p} \times \mathrm{M}_{p^{s+1}} .
$$

We have obtained the groups stated in part (e) of our theorem.

(i2b) Assume that $G / L$ is abelian of type $\left(p^{s}, p\right), s \geq 1$, and $K$ is abelian. Let $v \in K-L$ be such that $\langle v\rangle$ covers $K / L \cong \mathrm{C}_{p^{s}}, s \geq 1$. Then $t=v^{p^{s}} \in$ $U-H$ so that

$$
K / H \cong \mathrm{C}_{p^{s+1}} \text { and } K=H \times\langle v\rangle \cong \mathrm{E}_{p^{2}} \times \mathrm{C}_{p^{s+1}} .
$$


Since $G / L$ is abelian of type $\left(p^{s}, p\right)$, there is an element $w \in G-K$ such that $w^{p} \in L$ and so $w^{p} \in U$. Let $h \in H-U$ and consider the abelian subgroup

$$
\langle h, v\rangle \cong \mathrm{C}_{p} \times \mathrm{C}_{p^{s+1}}, s \geq 1 .
$$

Since $\langle h, v\rangle \unlhd G$, we get $\langle h, t\rangle \unlhd G$ and so $h^{w}=h t$ (where we replace $h$ with a suitable power $h^{j}, j \not \equiv 0(\bmod p)$, if necessary). In particular, we get $G^{\prime} \geq\langle t\rangle$.

Suppose that $G / U$ is nonabelian so that $p=2$ and $G / U$ is Hamiltonian. But $G / L$ is abelian and so

$$
(G / U)^{\prime}=\mho_{1}(G / U)=L / U
$$

Hence there is an element $m \in G$ such that $m^{2} \in L-U$, a contradiction. We have proved that $G / U$ is abelian and so $\langle t\rangle \leq G^{\prime} \leq U \leq \mathrm{Z}(G)$ and therefore $G$ is of class 2 with an elementary abelian commutator subgroup.

Note that

$$
\mathrm{C}_{p} \times \mathrm{C}_{p^{s+1}} \cong\langle h, v\rangle \unlhd G \text { and so }[h, w] \in\langle h, v\rangle \cap U=\langle t\rangle,
$$

which implies that $\langle v\rangle \unlhd G$ and therefore $p-1$ other cyclic maximal subgroups of $\langle h, v\rangle$ are also normal in $G$.

In case $\langle v\rangle \not \subset \mathrm{Z}(G)$ we get $v^{w}=v t^{j}$ for some integer $j \not \equiv 0(\bmod p)$. Solve the congruence $i j \equiv-1(\bmod p)$, where $i \not \equiv 0(\bmod p)$. Then we compute:

$$
\left(v^{i} h\right)^{w}=\left(v^{w}\right)^{i} h^{w}=\left(v t^{j}\right)^{i} h t=v^{i} t^{-1} h t=v^{i} h,
$$

where $\left\langle v^{i} h\right\rangle \cong \mathrm{C}_{p^{s+1}}$ is also a cyclic maximal subgroup in $\langle h, v\rangle$ and $\left\langle v^{i} h\right\rangle \leq$ $\mathrm{Z}(G)$. Thus replacing $\langle v\rangle$ with $\left\langle v^{i} h\right\rangle$, we may assume from the start that $\langle v\rangle \leq \mathrm{Z}(G)$. We get

$$
\mathrm{Z}(G)=\mathrm{C}_{K}(w)=\langle v\rangle U \text { and so }|G: \mathrm{Z}(G)|=p^{2} .
$$

By Lemma 1.1 in [1] we get

$$
|G|=p|\mathrm{Z}(G)|\left|G^{\prime}\right| \text { and so }\left|G^{\prime}\right|=p \text { and } G^{\prime}=\langle t\rangle \text {. }
$$

First suppose that $w^{p} \in U-\langle t\rangle$. Then $S=\langle h, w\rangle$ is the nonmetacyclic minimal nonabelian group of order $p^{4}$. If we set $Z=\langle v\rangle$, then we get

$$
G=Z * S, \text { where } Z \cong \mathrm{C}_{p^{s+1}} \text { and } Z \cap S=S^{\prime} .
$$

Assume that $w^{p} \in\langle t\rangle$ and set $\langle z\rangle=U \cap H$. Then $S=\langle h, w\rangle$ is isomorphic to $\mathrm{D}_{8}$ in case $p=2$ and to $\mathrm{S}\left(p^{3}\right)$ or $\mathrm{M}_{p^{3}}$ in case $p>2$. Setting again $Z=\langle v\rangle \cong \mathrm{C}_{p^{s+1}}$ we have $Z \leq \mathrm{Z}(G), S \cap Z=S^{\prime}$ and $G=\langle z\rangle \times(S * Z)$. However, in case $p>2$ and $S \cong \mathrm{M}_{p^{3}}$, we have $S * Z=S_{1} * Z$, where $S_{1} \cong \mathrm{S}\left(p^{3}\right)$ for a suitable subgroup $S_{1}$ in $S * Z$. We have obtained all groups stated in part (f) of our theorem.

(i2c) Assume that $G / L$ is abelian of type $\left(p^{s}, p\right), s \geq 1$, and $K$ is nonabelian. We have $K / L \cong \mathrm{C}_{p^{s}}, s \geq 1$. Let $v \in K-L$ be such that $\langle v\rangle$ covers $K / L$. Then $1 \neq t=v^{p^{s}} \in U-H$ so that $K / H \cong \mathrm{C}_{p^{s+1}}$. Acting with $K$ on $L$, we see that $K$ stabilizes the chain $L>U>\{1\}$. Hence if $s>1$, then there is 
an element $v_{0}$ of order $p^{2}$ in $K$ which centralizes $L$ and $v_{0}^{p} \in U-H$. For an element $h \in H-U$ we consider

$$
\mathrm{C}_{p} \times \mathrm{C}_{p^{2}} \cong\left\langle h, v_{0}\right\rangle \unlhd G \text { and so } \mathrm{E}_{p^{2}} \cong\left\langle h, v_{0}^{p}\right\rangle \unlhd G
$$

If $\left\langle h, v_{0}^{p}\right\rangle \not Z \mathrm{Z}(K)$, then there is an element $g \in G-K$ which centralizes $h$, a contradiction. Thus we must have $\left\langle h, v_{0}^{p}\right\rangle \leq \mathrm{Z}(K)$ and this implies that $K$ is abelian, a contradiction. We have proved that $s=1$ and so $t=v^{p}$ and $|G|=p^{5}$. Since $\mathrm{C}_{L}(v)=U=\mathrm{Z}(K)$, Lemma 1.1 in [1] gives that $\left|K^{\prime}\right|=p$. On the other hand, $K^{\prime} \leq H$ and since $K^{\prime} \leq \mathrm{Z}(G)$, we get $K^{\prime}=H \cap U$. For any $h \in H-U$, we have $\langle[h, v]\rangle=K^{\prime}$ and so $K$ is the nonmetacyclic minimal nonabelian group of order $p^{4}$ and $\Phi(K)=U$. Because $G / L \cong \mathrm{E}_{p^{2}}$, we have $\exp (G)=p^{2}$ and so for any $x \in G-L$, we have $x^{p} \in U$ and $\mho_{1}(G) \leq U$. For $p=2, G / U$ is elementary abelian. For $p>2$, the fact that $G / U$ is Dedekindian implies that $G / U$ is abelian and so again $G / U$ is elementary abelian. We have proved that $\Phi(G)=U$ and so $G^{\prime} \leq U$ and $\mathrm{d}(G)=3$. Since $\mathrm{Z}(G) \leq K$, we also get $\mathrm{Z}(G)=U$. If $G^{\prime}=K^{\prime}$, then $H \unlhd G$, a contradiction. Thus, $G^{\prime}=U$ and so $G$ is special.

By Lemma 146.7 in [4], $G$ has exactly one abelian maximal subgroup $A$ and for each subgroup $X_{i}$ of order $p$ in $G^{\prime}(i=1,2, \ldots, p+1)$ there are exactly $p$ pairwise distinct maximal subgroups $L_{i j}(j=1,2, \ldots, p)$ of $G$ such that $L_{i j}^{\prime}=X_{i}$.

Suppose that $G$ possesses a nonabelian subgroup $S$ of order $p^{3}$ so that $S$ is minimal nonabelian and $S \unlhd G$. But then $\mathrm{E}_{p^{2}} \cong G^{\prime} \leq S$ and since $G^{\prime}=\mathrm{Z}(G)$, we get that $S$ is abelian, a contradiction. Hence $G$ is an $\mathrm{A}_{2}$-group since each subgroup of index $p^{2}$ in $G$ is abelian and $K$ is a minimal nonabelian maximal subgroup in $G$. If there is an element $g \in G-K$ of order $p$, then $\langle g, h\rangle$ (with $h \in H-U)$ is minimal nonabelian of order $p^{3}$, a contradiction. We have proved that $\mathrm{E}_{p^{3}} \cong L=\Omega_{1}(G)$ and so a unique abelian maximal subgroup $A$ of $G$ is of type $\left(p^{2}, p^{2}\right)$. Indeed, $A$ contains $U=\Phi(G)$ and $|K \cap A|=p^{3}$. If $L \leq A$, then there is an element $g \in G-K$ which centralizes $L$, a contradiction. Hence we have $A \cap L=U=\Omega_{1}(A)$ which shows that $A \cong \mathrm{C}_{p^{2}} \times \mathrm{C}_{p^{2}}$.

By the results of $\S 71$ in [2], it follows that $G$ is one of $\mathrm{A}_{2}$-groups from Theorem 71.4(b2) in [2] with $\alpha=1$. We have obtained the groups from part (g) of our theorem.

(ii) We assume that whenever $H$ is a non-normal abelian subgroup of type $(p, p)$ in $G$, then $H \cap \mathrm{Z}(G)=\{1\}$. Let $z$ be a central element of $G$ which is contained in $L-H$ so that we have $L=\Omega_{1}(K)=\langle z\rangle \times H \cong \mathrm{E}_{p^{3}}$ and $L \cap \mathrm{Z}(G)=\langle z\rangle$. For any $1 \neq h \in H$, we have $\langle h, z\rangle \unlhd G$ and therefore $H \cap\langle h, z\rangle=\langle h\rangle \unlhd K$. Thus, $H \leq \mathrm{Z}(K)$ and $\mathrm{C}_{G}(L)=K$. It follows that $G / K$ acts faithfully on $L$ and stabilizes the chain $L>\langle z\rangle>\{1\}$ and $[H, G]=\langle z\rangle$. Thus $\{1\} \neq G / K$ is elementary abelian of order $\leq p^{2}$. However, if $|G / K|=p$, 
then there is an element $g \in G-K$ centralizing an element $1 \neq h \in H$ and so $h \in \mathrm{Z}(G)$, a contradiction. We have proved that we have $G / K \cong \mathrm{E}_{p^{2}}$.

Let $X$ be any cyclic subgroup of composite order in $G$. Since $\Omega_{1}(X) \leq K$, we have $\Omega_{1}(X) \leq L=\Omega_{1}(K)$. Suppose that $\Omega_{1}(X) \neq\langle z\rangle$. In this case we have

$$
X \times\langle z\rangle \unlhd G \text { and so } \Omega_{1}(X) \unlhd G \text {. }
$$

This is a contradiction since $L \cap \mathrm{Z}(G)=\langle z\rangle$. We have proved that the socle of each cyclic subgroup of composite order in $G$ is equal $\langle z\rangle \leq G^{\prime}$.

We have $\mathrm{Z}(G) \leq K$ and so we have

$$
\mathrm{Z}(G) \cap L=\mathrm{Z}(G) \cap \Omega_{1}(K)=\langle z\rangle .
$$

This implies that $\mathrm{Z}(G)$ is cyclic and we also have $|G: \mathrm{Z}(G)| \geq p^{4}$.

(ii1) First assume that $K / H \cong \mathrm{Q}_{8}$. In this case we have $|G|=2^{7}$. Let $K_{i}$ be any of the three maximal subgroups of $K$ containing $H$ so that $K_{i} / H \cong \mathrm{C}_{4}$ and therefore each $K_{i}$ is abelian. Hence $\left|K^{\prime}\right|=2$ and so $K^{\prime} \unlhd G$ and $K^{\prime} \leq L$ implies that $K^{\prime}=\langle z\rangle$. Let $v_{1}, v_{2} \in K-L$ be such that $\left\langle v_{1}, v_{2}\right\rangle$ covers $K / L$. Because $v_{1}^{2}=v_{2}^{2}=z$ and $\left[v_{1}, v_{2}\right]=z$, we get $Q=\left\langle v_{1}, v_{2}\right\rangle \cong \mathrm{Q}_{8}$ so that $K=H \times Q$ and $Q \unlhd G$. For each $K_{i}(i=1,2,3)$ we have $K_{i} \unlhd G$ and so $K_{i} \cap Q \unlhd G$. Thus $G$ induces on $Q$ only inner automorphisms of $Q$ which gives $G=Q * M$ with $Q \cap M=\langle z\rangle=Q^{\prime}$ and $M \cap K=L$, where $M=\mathrm{C}_{G}(Q)$ covers $G / K$. We have $\mho_{1}(M) \leq\langle z\rangle$ and so $Q /\langle z\rangle$ is elementary abelian. We get $G^{\prime}=\Phi(G)=\mathrm{Z}(G)=\langle z\rangle$ and so $G$ is extraspecial of order $2^{7}$. Since $M^{\prime}=\Phi(M)=\mathrm{Z}(M)=\langle z\rangle$, it follows that $M$ is extraspecial of order $2^{5}$ containing an elementary abelian subgroup $L$ of order 8 and so $M \cong \mathrm{Q}_{8} \times \mathrm{Q}_{8}$ and $G \cong \mathrm{Q}_{8} \times \mathrm{Q}_{8} \times \mathrm{Q}_{8}$. We have obtained the group stated in part (h) of our theorem.

(ii2) Assume that $K / H$ is cyclic. Then $K=H \times\langle v\rangle$ is abelian, where $\langle v\rangle \cong \mathrm{C}_{p^{s}}, s \geq 1$, and $\langle v\rangle \geq\langle z\rangle \leq G^{\prime} \cap \mathrm{Z}(G)$.

(ii2a) First suppose that $G^{\prime}=\langle z\rangle$. Then each cyclic subgroup of composite order is normal in $G$. Let $x, y \in G$ so that we have $\left[x^{p}, y\right]=[x, y]^{p}=1$ and therefore $\mho_{1}(G) \leq \mathrm{Z}(G)$. Hence we have $\Phi(G)=G^{\prime} \mho_{1}(G) \leq \mathrm{Z}(G)$ and we know that $\mathrm{Z}(G)$ is cyclic. Hence $\Phi(G)$ is also cyclic and $G^{\prime}=\Omega_{1}(\Phi(G))$. Since $v^{p} \in \mathrm{Z}(G)$, we have $|G: \mathrm{Z}(G)|=p^{4}$ or $p^{5}$. If $M$ is any minimal nonabelian subgroup in $G$, then either $M \cong \mathrm{S}\left(p^{3}\right)$ or $\mathrm{Z}(M)=\Phi(M)=\mho_{1}(M)$ and so in this case $M$ has a cyclic subgroup of index $p$. This gives:

$$
\begin{aligned}
& \text { If } p=2, \text { then } M \in\left\{\mathrm{D}_{8}, \mathrm{Q}_{8}, \mathrm{M}_{2^{n}}, n \geq 4\right\} . \\
& \text { If } p>2, \text { then } M \in\left\{\mathrm{S}\left(p^{3}\right), \mathrm{M}_{p^{n}}, n \geq 3\right\} .
\end{aligned}
$$

Let $A_{1}$ be any minimal nonabelian subgroup in $G$. Then we have $G=$ $A_{1} * C$, where $C=\mathrm{C}_{G}\left(A_{1}\right)$ with $A_{1} \cap C=\mathrm{Z}\left(A_{1}\right)$. If $C$ is abelian, then $C=\mathrm{Z}(G)$ and $|G: \mathrm{Z}(G)|=p^{2}$, a contradiction. Thus, $C$ is nonabelian and $\mathrm{Z}(C)=\mathrm{Z}(G)$, where $|C: \mathrm{Z}(C)|=p^{2}$ or $p^{3}$. Let $A_{2}$ be a minimal nonabelian 
subgroup in $C$. Then we have $C=A_{2} * C^{*}$, where $C^{*}=\mathrm{C}_{C}\left(A_{2}\right)$ and $A_{2} \cap C^{*}=$ $\mathrm{Z}\left(A_{2}\right)$. Note that $\mathrm{Z}\left(C^{*}\right)=\mathrm{Z}(C)$ and so if $C^{*}$ were nonabelian, then we get $\left|C^{*}: \mathrm{Z}\left(C^{*}\right)\right| \geq p^{2}$ and so $|C: \mathrm{Z}(C)| \geq p^{4}$, a contradiction. Hence $C^{*}$ is abelian and so $C^{*}=\mathrm{Z}(C)=\mathrm{Z}(G)$. We have proved that $G=A_{1} * A_{2} \mathrm{Z}(G)$, where $\mathrm{Z}(G)$ is cyclic. Finally, if $p=2$ and $A_{1} \cong \mathrm{Q}_{8}$ and $A_{2} \cong \mathrm{D}_{8}$, then we must have $|\mathrm{Z}(G)|>2$. Indeed, if we have in this case $|\mathrm{Z}(G)|=2$, then $G \cong \mathrm{Q}_{8} * \mathrm{D}_{8}$ and this group does not possess an elementary abelian subgroup of order 8. We have obtained the groups in part (i) of our theorem.

(ii2b) Finally assume that $G^{\prime}>\langle z\rangle$. Set $H=\left\langle h_{1}, h_{2}\right\rangle$ and we know that $\left\langle h_{1}, z\right\rangle \unlhd G,\left\langle h_{2}, z\right\rangle \unlhd G$ and both $G /\left\langle h_{1}, z\right\rangle$ and $G /\left\langle h_{2}, z\right\rangle$ are Dedekindian. If both $G /\left\langle h_{1}, z\right\rangle$ and $G /\left\langle h_{2}, z\right\rangle$ were abelian, then we get $G^{\prime} \leq\left\langle h_{1}, z\right\rangle \cap\left\langle h_{2}, z\right\rangle=$ $\langle z\rangle$, contrary to our assumption. Hence we must have $p=2$ and we may assume that $G /\left\langle h_{1}, z\right\rangle$ is Hamiltonian.

Let $Q /\left\langle h_{1}, z\right\rangle$ be an ordinary quaternion subgroup in $G /\left\langle h_{1}, z\right\rangle$ and set

$$
C /\left\langle h_{1}, z\right\rangle=\left(Q /\left\langle h_{1}, z\right\rangle\right)^{\prime}
$$

so that $Q^{\prime}$ covers $C /\left\langle h_{1}, z\right\rangle$. Since $G / K \cong \mathrm{E}_{4}$, we have $G^{\prime} \leq K$ and we know that $K$ is abelian. It follows that $C=\left\langle h_{1}, z\right\rangle Q^{\prime} \leq K$ and so $C$ is abelian of order 8. For each $x \in Q-C$ we have $x^{2} \in C-\left\langle h_{1}, z\right\rangle$. On the other hand, the socle of each cyclic subgroup of composite order in $G$ is equal $\langle z\rangle$ and so $o\left(x^{2}\right)=4$ and therefore $C$ is abelian of type $(4,2)$. We get $\Omega_{1}(Q)=\left\langle h_{1}, z\right\rangle, \Omega_{2}(Q)=C$, and all elements in $Q-C$ are of order 8. Also we have $Q \cap L=\left\langle h_{1}, z\right\rangle$. If $Q^{\prime}=C$, then $\left|Q: Q^{\prime}\right|=4$ and a well known result of O. Taussky would imply that $Q$ is of maximal class (and order $2^{5}$ ), contrary to the fact that $\Omega_{1}(Q)=\left\langle h_{1}, z\right\rangle \cong \mathrm{E}_{4}$. On the other hand, $Q^{\prime}$ must cover $C /\left\langle h_{1}, z\right\rangle$ and so we have $Q^{\prime} \cong \mathrm{C}_{4}$.

By Lemma 42.1 in [1], we have

$$
Q=\left\langle a, b \mid a^{8}=b^{8}=1, a^{4}=b^{4}=z, a^{b}=a^{-1}\right\rangle,
$$

where $Q^{\prime}=\left\langle a^{2}\right\rangle, \mathrm{Z}(Q)=\left\langle b^{2}\right\rangle, \Omega_{2}(Q)=\left\langle a^{2}, b^{2}\right\rangle$, and $\Omega_{1}(Q)=\left\langle z, a^{2} b^{2}\right\rangle$. Since $\mathrm{Z}(Q)=\left\langle b^{2}\right\rangle$, we have $\mathrm{C}_{Q}(b)=\langle b\rangle$ and so $\mathrm{C}_{\left\langle h_{1}, z\right\rangle}(b)=\langle z\rangle$. On the other hand, $b^{2} \in K>L$ and therefore $b^{2}$ centralizes $L$ and so $b$ induces an involutory automorphism on $L \cong \mathrm{E}_{8}$. Hence $\mathrm{C}_{L}(b) \cong \mathrm{E}_{4}$ and so there exists an involution $e \in H-\left\langle h_{1}\right\rangle$ such that $[e, b]=1$.

We have

$$
\mathrm{C}_{2} \times \mathrm{C}_{8} \cong\langle e, b\rangle \unlhd G, \text { where } \Omega_{1}(\langle e, b\rangle)=\langle e, z\rangle .
$$

On the other hand,

$$
b^{a}=a^{-1} b a=b\left(b^{-1} a^{-1} b\right) a=b a^{2},
$$

which shows that $a^{2} \in\langle e, b\rangle$. But then $\langle e, b\rangle$ contains $\left\langle e, z, a^{2} b^{2}\right\rangle \cong \mathrm{E}_{8}$, contrary to

$$
\Omega_{1}(\langle e, b\rangle)=\langle e, z\rangle \cong \mathrm{E}_{4} .
$$


We have proved that the case $G^{\prime}>\langle z\rangle$ cannot occur.

It remains to be proved the converse that all groups $G$ stated in our theorem satisfy the assumptions of that theorem. In fact, we have to prove that each noncyclic subgroup of order $\geq p^{3}$ is normal in $G$ and that $G$ has a non-normal abelian subgroup of type $(p, p)$.

If $G \cong \mathrm{D}_{16}$ or $G \cong \mathrm{SD}_{16}$, a four-subgroup in $G$ is not normal in $G$.

Let $G$ be a $p$-group in part (b) of our theorem. Then we have $L^{\prime}<G^{\prime}<L$, where $G^{\prime} \cong \mathrm{E}_{p^{2}}$. For an element $l \in L-G^{\prime}$, set $H=\left\langle L^{\prime}, l\right\rangle \cong \mathrm{E}_{p^{2}}$. If $H \unlhd G$, then $|G / H|=p^{2}$ implies that $G^{\prime} \leq H$, a contradiction. Hence $H$ is not normal in $G$.

Let $E$ be an elementary abelian maximal subgroup in a nonabelian $p$ group $G$ of order $p^{4}$ (from part (c) of our theorem). Then we have $1 \neq G^{\prime}<E$. Let $\mathrm{E}_{p^{2}} \cong H$ be any subgroup of order $p^{2}$ in $E$ which does not contain $G^{\prime}$. If $H \unlhd G$, then $|G / H|=p^{2}$ implies that $G^{\prime} \leq H$, a contradiction. Hence $H$ is not normal in $G$.

Let $G$ be a 2 -group of order $2^{6}$ from part (d) of our theorem. Note that $\mathrm{Z}(G) \cong \mathrm{E}_{4}$ implies that $G$ has no abelian maximal subgroup. Indeed, if $G$ would have an abelian maximal subgroup, then we may use Lemma 1.1 in [1] and we get

$$
|G|=2^{6}=2\left|G^{\prime}\right||\mathrm{Z}(G)|=2^{3}\left|G^{\prime}\right| \text { and }\left|G^{\prime}\right|=2^{3},
$$

which contradicts the fact that $\left|G^{\prime}\right|=2$. Let $S$ be a noncyclic subgroup of order $\geq 2^{3}$ and assume that $S$ is not normal in $G$. Then $G^{\prime} \not \leq S$ and so $S$ is noncyclic abelian. If $|S|=2^{4}$, then $S \times G^{\prime}$ would be an abelian maximal subgroup of $G$, a contradiction. Assume that $|S|=2^{3}$. Since $G$ has no elementary abelian subgroups of order $2^{4}$, we get that $S$ is abelian of type $(4,2)$. In case $G \cong\left(\mathrm{D}_{8} * \mathrm{Q}_{8}\right) \times \mathrm{C}_{2}$, we have $\mho_{1}(G)=G^{\prime}$ and so $\left(\right.$ since $G^{\prime} \not \leq S$ ) we must be in case

$\mathrm{H}_{16} * \mathrm{Q}_{8} \cong G=D * Q$, where $D \cong \mathrm{H}_{16}, Q \cong \mathrm{Q}_{8}$ and $D \cap Q=D^{\prime}=\langle z\rangle=Q^{\prime}$, and $z$ is not a square of any element in $D$. Since all elements in $G-D$ are of order 4 , we have $\Omega_{1}(S) \leq D$ and so

$$
\mathrm{E}_{8} \cong \Omega_{1}(D)=\Omega_{1}(S) \times D^{\prime}=\Omega_{1}(S) \times\langle z\rangle .
$$

We have

$$
\begin{gathered}
\mathrm{C}_{D}\left(\Omega_{1}(S)\right)=\Omega_{1}(S) \times\langle z\rangle=\Omega_{1}(D) \text { and } \mathrm{C}_{G}\left(\Omega_{1}(S)\right)=\Omega_{1}(D) * Q, \\
\text { where } \mho_{1}\left(\mathrm{C}_{G}\left(\Omega_{1}(S)\right)\right)=\langle z\rangle .
\end{gathered}
$$

But $S \leq \mathrm{C}_{G}\left(\Omega_{1}(S)\right)$ and so $G^{\prime}=\langle z\rangle \leq S$, a contradiction. It is easy to see that $G$ possesses a non-normal abelian subgroup $H \cong \mathrm{E}_{4}$. Set $H=\langle t, u\rangle$, where $t$ is a noncentral involution in $G$ and $u$ is a central involution in $G$ such that $\langle u\rangle \neq G^{\prime}$. Then we have $G^{\prime} \not \leq H$. If $H \unlhd G$, then there is $g \in G$ such 
that $[g, t] \neq 1$ and so $G^{\prime}=\langle[g, t]\rangle \leq H$, a contradiction. Hence $H=\langle t, u\rangle$ is not normal in $G$.

Let $G=M \times\langle t\rangle$, where $M \cong \mathrm{M}_{p^{s+1}}, s \geq 3$, and $\langle t\rangle \cong \mathrm{C}_{p}$ (which are groups of part (e) of our theorem). We have $\Omega_{1}(G) \cong \mathrm{E}_{p^{3}}, \Omega_{2}(G)$ is abelian of type $\left(p^{2}, p, p\right)$ with $\mho_{1}\left(\Omega_{2}(G)\right)=G^{\prime}=\mathrm{C}_{p}$. Thus any subgroup of order $\geq p^{3}$ is normal in $G$. Let $H$ be a complement of $G^{\prime}$ in $\Omega_{1}(G)$ so that $H \not Z \mathrm{Z}(G)$ and so $H$ is not normal in $G$. Indeed, if in this case $H \unlhd G$, then $[G, H] \neq\{1\}$ and $[G, H] \leq H$ and so $G^{\prime} \leq H$, a contradiction.

Let $G$ be a group of part (f) of our theorem. Let $X$ be any subgroup of $G$ of order $\geq p^{3}$ which is not normal in $G$. Then we have $G^{\prime}=S^{\prime} \not \leq X$ and so $X$ is abelian of order $\geq p^{3}$ with $X \cap Z=\{1\}$. But $|G / Z|=p^{3}$ and so $|X|=p^{3}$ and $G=Z \times X$ is abelian, a contradiction. Let $H=\langle t, u\rangle \cong \mathrm{E}_{p^{2}}$, where $t$ is a noncentral element of order $p$ in $S$ and $u$ is a central element of order $p$ in $G$ with $\langle u\rangle \neq G^{\prime}$. Then we have $G^{\prime} \not \leq H$ and so $H$ is not normal in $G$.

Let $G$ be a group of order $p^{5}$ given in part (g) of our theorem. Then $G$ is special with $G^{\prime} \cong \mathrm{E}_{p^{2}}$ and $G$ is an $\mathrm{A}_{2}$-group. Let $Y$ be any subgroup of $G$ of order $p^{3}$ which does not contain $G^{\prime}$. Since $|G: Y|=p^{2}$ and $G$ is an $\mathrm{A}_{2}$-group, it follows that $Y$ is abelian of type $\left(p^{2}, p\right)$. Then $A=G^{\prime} Y$ is a unique abelian maximal subgroup of $G$ and we know that $A \cong \mathrm{C}_{p^{2}} \times \mathrm{C}_{p^{2}}$. But then $\mathrm{E}_{p^{2}} \cong \Omega_{1}(A)=\Phi(A)=G^{\prime}$, a contradiction. Let $H$ be an abelian subgroup of order $p^{2}$ contained in $\Omega_{1}(G) \cong \mathrm{E}_{p^{3}}$ distinct from $G^{\prime}$. If $H \unlhd G$, then $G=H A$ and $G / H$ is abelian so that $G^{\prime} \leq H$, a contradiction. Hence $H$ is not normal in $G$.

Let $G \cong \mathrm{Q}_{8} * \mathrm{Q}_{8} * \mathrm{Q}_{8}$ be the extraspecial group of order $2^{7}$ given in part (h) of our theorem. Let $X$ be any subgroup of order $\geq 2^{3}$ and assume that $X$ is not normal in $G$. Then $X \cap G^{\prime}=\{1\}$ and so $X$ is elementary abelian. But then $X \times G^{\prime}$ is an elementary abelian subgroup of order $\geq 2^{4}$ in $G$. Since $G$ is extraspecial of order $2^{7}$ and type " - ", there are no such elementary abelian subgroups in $G$. Hence $X \unlhd G$. Let $H$ be a four-subgroup in $G$ with $H \cap G^{\prime}=\{1\}$. If $H \unlhd G$, then $H \cap \mathrm{Z}(G) \neq\{1\}$, a contradiction.

Finally, let $G$ be a group stated in part (i) of our theorem. Then we have

$$
\Omega_{1}(\mathrm{Z}(G))=G^{\prime} \text {, where } \mathrm{Z}(G) \text { is cyclic. }
$$

Also note that $|G: \mathrm{Z}(G)|=p^{4}$ and so $G$ does not possess an abelian maximal subgroup. Indeed, if $G$ would have an abelian maximal subgroup, then Lemma 1.1 in [1] implies that

$$
|G|=p\left|G^{\prime}\right||\mathrm{Z}(G)|, \text { where }\left|G^{\prime}\right|=p,
$$

a contradiction. Let $X$ be any subgroup of order $\geq p^{3}$ in $G$. Then we claim that $X \unlhd G$. Indeed, assume that $X$ is not normal in $G$. Then we have $G^{\prime} \not \leq X$ and so $X \cap \mathrm{Z}(G)=\{1\}$ and therefore $X$ is abelian of order $\geq p^{3}$. But then $\mathrm{Z}(G) \times X$ is an abelian subgroup of index $\leq p$ in $G$, a contradiction. It remains to be shown that $G=\left(A_{1} * A_{2}\right) \mathrm{Z}(G)$ possesses an abelian subgroup of type 
$(p, p)$ which is not normal in $G$. If $A_{1}$ and $A_{2}$ possess noncentral elements $a_{1} \in A_{1}$ and $a_{2} \in A_{2}$ of order $p$, then $H=\left\langle a_{1}, a_{2}\right\rangle \cong \mathrm{E}_{p^{2}}$ and $H$ is not normal in $G$ since $H \cap \mathrm{Z}(G)=\{1\}$. If $p>2$, then

$$
A_{1}, A_{2} \in\left\{\mathrm{S}\left(p^{3}\right), \mathrm{M}_{p^{n}}, n \geq 3\right\}
$$

and in this case there are such elements $a_{1}$ and $a_{2}$. If $p=2$, then we have

$$
A_{1}, A_{2} \in\left\{\mathrm{D}_{8}, \mathrm{Q}_{8}, \mathrm{M}_{2^{n}}, n \geq 4\right\}
$$

and we may replace $A_{1}$ and $A_{2}$ with suitable other minimal nonabelian subgroups of $G$ so that again we find noncentral involutions $a_{1} \in A_{1}$ and $a_{2} \in A_{2}$. Indeed we have:

$$
\begin{gathered}
\mathrm{Q}_{8} * \mathrm{Q}_{8}=\mathrm{D}_{8} * \mathrm{D}_{8}, \\
\mathrm{Q}_{8} * \mathrm{M}_{2^{n}}=\mathrm{D}_{8} * \mathrm{M}_{2^{n}}, n \geq 4,
\end{gathered}
$$

and

$$
\left(\mathrm{D}_{8} * \mathrm{Q}_{8}\right) \mathrm{Z}(G)=\left(\mathrm{D}_{8} * \mathrm{D}_{8}\right) \mathrm{Z}(G) \text {, where }|\mathrm{Z}(G)|>2 .
$$

Theorem A is completely proved.

\section{Proof of Theorem B}

First we shall prove a series of lemmas about 2-groups $G$ which satisfy the assumptions of Theorem $\mathrm{B}$, where $H$ always denotes a non-normal subgroup in $G$ which is isomorphic to Q8. Set $K=\mathrm{N}_{G}(H)$ so that $H<K<G$ and $K \unlhd G$. Let $L$ be a unique subgroup in $G$ which contains $H$ as a subgroup of index 2 . We fix this notation in the sequel.

Lemma 3.1. The factor-group $K / H \neq\{1\}$ is either cyclic or isomorphic to $\mathrm{Q}_{8}$ and $G / L \neq\{1\}$ is Dedekindian. We have $\Omega_{1}(K) \leq L$ and if $K$ does not possess a $G$-invariant four-subgroup, then $G \cong \mathrm{Q}_{2^{5}}$ (the case (a) of Theorem $B)$. From now on we shall assume that $K$ possesses a $G$-invariant foursubgroup $U$. We have in that case $L=H U$ with $U_{0}=H \cap U=\mathrm{Z}(H) \leq \mathrm{Z}(G)$ and $G / U$ is also Dedekindian.

Proof. Since $K / H$ is Dedekindian and $L / H$ is a unique subgroup of order 2 in $K / H$, it follows that $K / H \neq\{1\}$ is either cyclic or isomorphic to Q8 which also implies that $\Omega_{1}(K) \leq L$.

Assume that $K$ has no $G$-invariant four-subgroup. By Lemma 1.4 in [1], $K$ is a 2-group of maximal class and then $K=L$ is of order $2^{4}$. We have $\mathrm{C}_{G}(H)=\mathrm{C}_{K}(H)<H$ and then Proposition 10.17 in [1] implies that $G$ is also of maximal class. Since $K \unlhd G$, we must have $|G / K|=2$ and so $|G|=2^{5}$. The only possibility is $G \cong \mathrm{Q}_{2^{5}}$ and this group obviously satisfies the assumptions of Theorem B.

From now on we shall assume that $K$ has a $G$-invariant four-subgroup $U$. Since $\Omega_{1}(K) \leq L$, we have $U \leq L$ and so $L=H U$ with $U_{0}=H \cap U=\mathrm{Z}(H)$. But $L^{\prime} \leq H \cap U$ and so we have $L^{\prime}=U_{0} \leq \mathrm{Z}(G)$. Also, $G / U$ is Dedekindian. 
Lemma 3.2. We have $U=\mathrm{Z}(L) \leq G^{\prime}, K=H * \mathrm{C}_{G}(H)$ with $U \leq \mathrm{C}_{G}(H)$ and $H \cap \mathrm{C}_{G}(H)=U_{0}$. Also, $G / K$ is elementary abelian of order 2 or 4 and $\Omega_{1}(K)=U$.

Proof. Since $L^{\prime}=H^{\prime}=U_{0}$, we get $L=H * Z$, where $Z \cong \mathrm{C}_{4}$ or $\mathrm{E}_{4}$ and $H \cap Z=U_{0}$. However, if $Z \cong \mathrm{C}_{4}$, then $H$ would be a unique subgroup in $L$ which is isomorphic to $\mathrm{Q}_{8}$ and this gives $H \unlhd G$, a contradiction. Hence we have $Z \cong \mathrm{E}_{4}$ and so

$$
U=\Omega_{1}(L)=\Omega_{1}(K)=\mathrm{Z}(L) .
$$

Let $H_{1}$ be any cyclic subgroup of order 4 in $H$. Then

$$
H_{1} U \unlhd G \text { and so } H_{1}=\left(H_{1} U\right) \cap H \unlhd K .
$$

Thus each element in $K$ induces on $H$ an inner automorphism of $H$ and so we get

$$
K=H * \mathrm{C}_{G}(H) \text { with } U \leq \mathrm{C}_{G}(H) \text { and } H \cap \mathrm{C}_{G}(H)=U_{0} .
$$

For an element $x \in G-K$, there is an element $h \in H$ of order 4 such that $h^{x} \in L-H$. But $\langle h\rangle U \unlhd G$ with $h^{2} \in U_{0}$ and so $h^{x}=h u$ for some $u \in U-U_{0}$. Then we have $[h, x]=u$ and so we get $U \leq G^{\prime}$.

There are exactly three maximal subgroups of $L$ which contain $U$ and they all are abelian of type $(4,2)$. The other four maximal subgroups of $L$ which do not contain $U$ are isomorphic to $\mathrm{Q}_{8}$. This gives $1 \neq|G / K| \leq 4$.

For any element $y \in H-U_{0}$ and any $g \in G-K$, we have

$$
y^{2} \in U_{0}, U\langle y\rangle \unlhd G \text { and } y^{g}=y u, \text { where } u \in U .
$$

This gives

$$
y^{g^{2}}=(y u)^{g}=(y u) u^{g}=(y u) u u_{0}=y u_{0} \text { with some } u_{0} \in U_{0} .
$$

Hence $g^{2} \in K$ and so $G / L$ is elementary abelian of order $\leq 4$.

LEMmA 3.3. If $U \not z \mathrm{Z}(G)$, then $G$ is the group of order $2^{5}$ and class 3 from part (b) of Theorem $B$ and this group satisfies the assumptions of that theorem.

Proof. Assume that $U \not Z \mathrm{Z}(G)$. Note that $K / H \cong \mathrm{C}_{G}(H) / U_{0}$ is either cyclic or isomorphic to $\mathrm{Q}_{8}$. Hence if $K>L$, then $\mathrm{C}_{G}(H)=\mathrm{C}_{K}(H)>U$ and so there is an element $k$ of order 4 in $\mathrm{C}_{K}(H)-U$ such that $k^{2} \in U-U_{0}$. In that case we have

$$
U\langle k\rangle=U_{0} \times\langle k\rangle \cong \mathrm{C}_{2} \times \mathrm{C}_{4} \unlhd G .
$$

But then we get $\left\langle k^{2}\right\rangle \unlhd G$ and so $U \leq \mathrm{Z}(G)$, a contradiction.

We have proved that $K=L$. Suppose that $G-K$ contains an element $y$ of order $\leq 4$ which does not centralize $U$. Since $y^{2} \in U$, we get $D=U\langle y\rangle \cong$ 
$\mathrm{D}_{8} \unlhd G$. Let $V$ be a four-subgroup in $D$ which is distinct from $U$. Because $U \unlhd G$, we get also $V \unlhd G$ and $V \cap K=U_{0}=\mathrm{Z}(D)$. But then we have

$$
[H, V] \leq K \cap V=U_{0}<H
$$

and so $V$ normalizes $H$, a contradiction. Hence each element in $G-K$ of order $\leq 4$ centralizes $U$ and since $U \not \mathbf{Z}(G)$, there is an element $x$ of order 8 in $G-K$ so that we have $x^{2} \in L-U$ and $\left\langle x^{4}\right\rangle=U_{0}$. Note that $\langle x\rangle U \unlhd G$ and we have either $\langle x\rangle U \cong \mathrm{C}_{8} \times \mathrm{C}_{2}$ or $\langle x\rangle U \cong \mathrm{M}_{16}$. In any case $\left\langle x^{2}\right\rangle$ is characteristic in $\langle x\rangle U$ and so $\left\langle x^{2}\right\rangle \unlhd G$. Then there are exactly three maximal subgroups of $K=L$ which contain $\left\langle x^{2}\right\rangle$, where two of them are isomorphic to $\mathrm{Q}_{8}$ and $\left\langle x^{2}\right\rangle U \cong \mathrm{C}_{4} \times \mathrm{C}_{2}$. Thus acting with $G / K$ on four maximal subgroups of $L$ which are isomorphic to $\mathrm{Q}_{8}$, we get $|G: K|=2$ and so $|G|=2^{5}$. Since $U \leq \mathrm{Z}(K)$ (noting that $K=L$ ), each element in $G-K$ does not centralize $U$ and so (by the above argument) all elements in $G-K$ are of order 8 .

We have proved that $\Omega_{2}(G)=K=L \cong \mathrm{C}_{2} \times \mathrm{Q}_{8}$ and so by Theorem 52.1 in [2], $G$ is isomorphic to the group defined in part A2(a) of Theorem 49.1 in [2]. Since $\Omega_{1}(G)=G^{\prime}=U$, this group obviously satisfies the assumptions of Theorem $\mathrm{B}$ and we are done.

From now on we shall always suppose that $U \leq \mathrm{Z}(G)$.

LEMMA 3.4. The factor-group $G / U$ is abelian and so we have $G^{\prime}=U \leq$ $\mathrm{Z}(G)$. Since for all $x, y \in G$ we get $\left[x^{2}, y\right]=[x, y]^{2}=1$, it follows that $\Phi(G) \leq \mathrm{Z}(G)$.

Proof. Assume that $G / U$ is nonabelian so that $G / U$ is Hamiltonian. Let $Q / U$ be an ordinary quaternion subgroup in $G / U$, where by our assumption we have $U \leq \mathrm{Z}(G)$ (see Lemma 3 ). Set

$$
Q_{0} / U=(Q / U)^{\prime}=\mathrm{Z}(Q / U), \text { where }\left|Q_{0}: U\right|=2 .
$$

Let $Q_{1} / U$ and $Q_{2} / U$ be two distinct cyclic subgroups of order 4 in $Q / U$ so that $Q_{1}$ and $Q_{2}$ are two distinct abelian maximal subgroups in $Q$. This implies that $\left|Q^{\prime}\right|=2$. On the other hand, $Q^{\prime}$ covers $Q_{0} / U=(Q / U)^{\prime}$ and so $Q_{0}=U \times Q^{\prime} \cong \mathrm{E}_{8}$. For each $l \in Q-Q_{0}$, we have $l^{2} \in Q_{0}-U$ and $l^{2} \in K$ (since $G / K$ is elementary abelian of order $\leq 4$ ). But then $Q_{0} \leq K$ which contradicts Lemma 2 which states that $\Omega_{1}(\bar{K})=U$.

Lemma 3.5. There are no involutions in $G-K$ and so we have $U=G^{\prime}=$ $\Omega_{1}(G) \leq \mathrm{Z}(G)$.

Proof. Set $\mathrm{Z}(H)=H^{\prime}=\langle z\rangle$ and suppose that there is an involution $i$ in $G-K$. Then $H \neq H^{i}$ and $i$ normalizes $H_{0}=H \cap H^{i} \cong \mathrm{C}_{4}$. It follows that $H_{0}\langle i\rangle \cong \mathrm{C}_{4} \times \mathrm{C}_{2}$ or $\mathrm{D}_{8}$ and $H_{0}\langle i\rangle \unlhd G$. If $\langle z, i\rangle$ is not normal in $G$, then $H_{0}\langle i\rangle \cong \mathrm{D}_{8}$ and there is $g \in G$ which induces on $H_{0}\langle i\rangle$ an outer automorphism (which permutes two four-subgroups in $H_{0}\langle i\rangle$ ). But in that case we have $\left[\left(H_{0}\langle i\rangle\right),\langle g\rangle\right]=H_{0} \cong \mathrm{C}_{4}$, contrary to the fact that $G^{\prime}=U \cong \mathrm{E}_{4}$. It follows 
that we have $E=\langle z, i\rangle \unlhd G$. But then we have $[H, E] \leq K \cap E=\langle z\rangle$ and so $i$ normalizes $H$, a contradiction.

Lemma 3.6. The factor-group $K / H$ is cyclic.

Proof. Assume that $K / H$ is noncyclic so that setting $\mathrm{Z}(H)=H^{\prime}=\langle z\rangle$ we get

$$
\mathrm{Q}_{8} \cong K / H \cong \mathrm{C}_{G}(H) /\langle z\rangle
$$

and therefore

$$
\mathrm{Z}\left(\mathrm{C}_{G}(H)\right)=U \text { and } \mathrm{Z}(K)=U=\mathrm{Z}(G) .
$$

By Lemma 4, we have $\Phi(G) \leq \mathrm{Z}(G)$ and so $\Phi(G)=U$. On the other hand, $|K|=2^{6}$ and so $|G| \geq 2^{7}$ and $\mathrm{d}(G) \geq 5$. By Lemma $5, G$ has no normal elementary abelian subgroup of order 8 and so by the four-generator theorem (see Theorem 50.3 in [2]), we must have $\mathrm{d}(G) \leq 4$, a contradiction.

Proof of Theorem B. We continue with the situation which we have reached after Lemma 6 . Hence we have

$$
\begin{gathered}
U=G^{\prime}=\Omega_{1}(G) \leq \mathrm{Z}(G), \Phi(G) \leq \mathrm{Z}(G), \\
K=H \times\langle a\rangle \text { with }\langle a\rangle \cong \mathrm{C}_{2^{n}}, n \geq 1, L=H \times \Omega_{1}(\langle a\rangle),
\end{gathered}
$$

and $G / K \neq\{1\}$ is elementary abelian of order $\leq 4$.

(i) First assume $K=L$. In this case $G$ is a special group of order $2^{5}$ or $2^{6}$ with

$$
\Omega_{1}(G)=\Phi(G)=\mathrm{Z}(G)=G^{\prime}=U \cong \mathrm{E}_{4} \text { and we set } \mathrm{Z}(H)=\langle z\rangle .
$$

Let $G_{0} / K$ be any fixed subgroup of order 2 in $G / K$ and let $x \in G_{0}-K$. Then $x$ normalizes

$$
H_{0}=\left\langle h_{0}\right\rangle=H \cap H^{x} \cong \mathrm{C}_{4} .
$$

If $x$ inverts $h_{0}$, then for an element $h \in H-H_{0}$, we have $h x \in G_{0}-K$ and $h x$ centralizes $H_{0}$. Hence there is an element $v \in G_{0}-K$ such that $v$ centralizes an element $h_{0} \in H$ of order 4. If $v^{2}=z$, then $h_{0} v$ is an involution in $G-K$, a contradiction. Hence we have $v^{2}=z^{\prime} \in U-\langle z\rangle$. Since $H$ is not normal in in $G_{0}$, we have for any $h_{1} \in H-\left\langle h_{0}\right\rangle,\left[h_{1}, v\right] \in\left\{z^{\prime}, z z^{\prime}\right\}$. However, if $\left[h_{1}, v\right]=z z^{\prime}$, then we get

$$
\left(h_{1} v\right)^{2}=h_{1}^{2} v^{2}\left[h_{1}, v\right]=z z^{\prime}\left(z z^{\prime}\right)=1,
$$

and so $h_{1} v$ is an involution in $G-K$, a contradiction. Thus we get $\left[h_{1}, v\right]=$ $z^{\prime}=v^{2}$ and so $\langle v\rangle \unlhd G_{0}$. It follows that $G_{0}$ is a splitting extension of the cyclic noncentral normal subgroup $\langle v\rangle$ of order 4 (with $v^{2}=z^{\prime}$ ) by $H \cong \mathrm{Q}_{8}$. We have obtained the group stated in part (c) of Theorem B. Note that $\left(h_{0} v\right)^{2}=z z^{\prime},\left\langle h_{0} v\right\rangle$ centralizes $\left\langle h_{0}\right\rangle$ and $\left[h_{1}, h_{0} v\right]=z z^{\prime}$ and so $G_{0}$ is also a splitting extension of the cyclic noncentral normal subgroup $\left\langle h_{0} v\right\rangle$ of order 4 (with $\left(h_{0} v\right)^{2}=z z^{\prime}$ ) by $H \cong \mathrm{Q}_{8}$. 
Suppose now in addition that we have $G / K \cong \mathrm{E}_{4}$. If a cyclic subgroup $\langle h\rangle$ of order 4 in $H$ is normal in $G$, then acting with $G / K$ on four quaternion subgroups in $K=L$, we see that $G$ interchanges two quaternion subgroups which contain $\langle h\rangle$ and so $G$ interchanges also the other two quaternion subgroups in $K$. But this implies that $|G / K|=2$, a contradiction. Hence if $G_{i} / K$ are three subgroups of order 2 in $G / K, i=1,2,3$, then each $G_{i}$ normalizes exactly one of the three cyclic subgroups of order 4 in $H$. This implies that that there is an element $w \in G-G_{0}$ such that $w$ centralizes $h_{1}$ (from the previous paragraph), $w^{2}=z^{\prime}$ and $\left[h_{0}, w\right]=z^{\prime}$ so that $K\langle w\rangle$ is a splitting extension of the cyclic noncentral normal subgroup $\langle w\rangle$ of order 4 (with $w^{2}=z^{\prime}$ ) by $H \cong \mathrm{Q}_{8}$. We have

$$
\left[h_{0}, v w\right]=z^{\prime},\left[h_{1}, v w\right]=z^{\prime},\left[h_{0} h_{1}, v w\right]=1,
$$

and so $H$ normalizes $\langle v w\rangle$ with $H \cap\langle v w\rangle=\{1\}$. By the above, we must have $(v w)^{2}=z^{\prime}$ and so we have

$$
z^{\prime}=(v w)^{2}=v^{2} w^{2}[v, w]=z^{\prime} z^{\prime}[v, w]=[v, w],
$$

which implies that $\langle v, w\rangle \cong \mathrm{Q}_{8}$ with $\mathrm{Z}(\langle v, w\rangle)=\left\langle z^{\prime}\right\rangle$. But $H$ normalizes both $\langle v\rangle$ and $\langle w\rangle$ and so $H_{1}=\langle v, w\rangle \unlhd G$. The structure of $G$ is uniquely determined. We verify that we have also $H_{2}=\left\langle h_{1} w, h_{0} v\right\rangle \cong \mathrm{Q}_{8}$ with $\mathrm{Z}\left(\left\langle h_{1} w, h_{0} v\right\rangle\right)=\left\langle z z^{\prime}\right\rangle$ and $\left[H_{1}, H_{2}\right]=\{1\}$. Since $H_{1} \cap H_{2}=\{1\}$, we have obtained the group $G=H_{1} \times H_{2}$ from part (d) of Theorem B.

Finally, in both cases of groups $G$ in parts (c) and (d) of Theorem B, we have $\Omega_{1}(G)=G^{\prime} \cong \mathrm{E}_{4}$ and so if $X$ is any subgroup in $G$ of order $\geq 2^{3}$ and if $X$ contains only one involution, then $X \cong \mathrm{Q}_{8}$ and if $X$ contains more than one involution, then $X \geq G^{\prime}$ and so $X \unlhd G$. Thus in both cases the assumptions of Theorem B are satisfied.

(ii) Now assume that $K>L$ and so $\left|\mathrm{C}_{G}(H): U\right| \geq 2$. Since $G / L$ is abelian, $G / K$ is elementary abelian of order 2 or 4 , and $K / L$ is cyclic of order $\geq 2$, we have to consider two subcases.

(ii1) $G / K$ has a subgroup $G_{0} / K$ of order 2 such that $G_{0} / L$ is cyclic of order $\geq 4$ and either $G=G_{0}$ or $G=G_{0} G_{1}$ with $G_{0} \cap G_{1}=L$ and $\left|G_{1}: L\right|=2$. We set $\mathrm{Z}(H)=\langle z\rangle$. Let $g$ be an arbitrary element in $G_{0}-K$ so that $\langle g\rangle$ covers $G_{0} / L$. Since $g^{2} \in \mathrm{Z}(G)$, we have $g^{2} \in \mathrm{C}_{G}(H)$. Because $K / H$ is cyclic but $U \leq \mathrm{C}_{G}(H)$ is noncyclic and $\mathrm{C}_{G}(H) /\langle z\rangle \cong \mathrm{K} / \mathrm{H}$, we get $\mathrm{C}_{G}(H)=\langle z\rangle \times\left\langle g^{2}\right\rangle$ with $o\left(g^{2}\right) \geq 4$ and so $o(g) \geq 8$. Let $\left\langle z^{\prime}\right\rangle=\Omega_{1}(\langle g\rangle)$ be the socle of $\langle g\rangle$, where $U=\left\langle z, z^{\prime}\right\rangle$. We have

$$
H_{0}=\left\langle h_{0}\right\rangle=H \cap H^{g} \cong \mathrm{C}_{4}
$$

is $\langle g\rangle$-invariant and so $H_{0} \unlhd G_{0}$. But $h_{1} \in H-H_{0}$ inverts $\left\langle h_{0}\right\rangle$ and so $\mathrm{C}_{G}\left(h_{0}\right)$ covers $G_{0} / K$. Therefore we may choose $g \in \mathrm{C}_{G}\left(h_{0}\right)-K$ so that we may assume $\left[g, h_{0}\right]=1$. But $H$ is not normal in $G_{0}$ and so $\left[h_{1}, g\right] \in\left\{z^{\prime}, z z^{\prime}\right\}$ and we may set $\left[h_{1}, g\right]=z^{\epsilon} z^{\prime}$, where $\epsilon=0,1$. We have obtained the groups 
from part (e) of Theorem B which obviously satisfy the assumptions of that theorem.

Continuing with this case, we assume that $G=G_{0} G_{1}$ with $G_{0} \cap G_{1}=$ $L=H U$ and $\left|G_{1}: L\right|=2$. The group $G_{1}$ is isomorphic to a group in part (c) of Theorem B and so there is an element $v \in G_{1}-L$ of order 4 such that $v^{2}=z^{\prime}$ and $H$ normalizes but does not centralize $\langle v\rangle$ (see arguments in (i)). On the other hand, $g^{2} \in \mathrm{Z}(G)$ and $o\left(g^{2}\right) \geq 4$ and so there is an element $w$ of order 4 in $\left\langle g^{2}\right\rangle$. But then $v w$ is an involution in $G-K$, contrary to Lemma 5 .

(ii2) $G=K G^{*}$, where $K \cap G^{*}=L$ and $G^{*} / L$ is elementary abelian of order 2 or 4 . Also we have $K=H \times\langle a\rangle$, where $o(a) \geq 4$. Also we set $\mathrm{Z}(H)=\langle z\rangle$ and $\Omega_{1}(\langle a\rangle)=\left\langle z^{\prime}\right\rangle$ so that $U=\left\langle z, z^{\prime}\right\rangle$. In any case, we have in $G^{*}-L$ an element $v$ of order 4 such that $v^{2}=z^{\prime}$ and $H$ normalizes but does not centralize $\langle v\rangle$. We have $\mathrm{Z}(G) \leq \mathrm{C}_{G}(H)=U\langle a\rangle$. If $\mathrm{Z}(G)>U$, then there is an element $w$ of order 4 in $\langle a\rangle$ with $w^{2}=z^{\prime}$ and $[v, w]=1$. But then $v w$ is an involution in $G-K$, contrary to Lemma 5 .

We have proved that $\Omega_{1}(G)=\mathrm{Z}(G)=U$ and so, in particular, $o(a)=4$ and $a \notin \mathrm{Z}(G)$. This also gives that $\exp (G)=4$ (because $\mho_{1}(G) \leq \mathrm{Z}(G)$ ). Hence $G$ is a special group of order $2^{6}$ or $2^{7}$. But $G$ has no normal elementary abelian subgroup of order 8 and so by the four-generator theorem we must have $\mathrm{d}(G) \leq 4$. Since $\Phi(G)=U$, we must have $|G|=2^{6}$ and $\left|G^{*}: L\right|=2$. We may set $H=\left\langle h_{0}, h_{1}\right\rangle$ so that $\left[h_{0}, v\right]=1$ and $\left[h_{1}, v\right]=z^{\prime}$. Set $[a, v]=u$, where $1 \neq u \in U$. We compute:

$$
\begin{aligned}
& (v a)^{2}=v^{2} a^{2} u=z^{\prime} z^{\prime} u=u \neq 1, \\
& \left(v\left(a h_{0}\right)\right)^{2}=z^{\prime}\left(z z^{\prime}\right) u=u z \text { and so } u \neq z, \\
& \left(v\left(a h_{1}\right)\right)^{2}=z^{\prime}\left(z z^{\prime}\right) u z^{\prime}=u\left(z z^{\prime}\right) \text { and so } u \neq z z^{\prime} .
\end{aligned}
$$

It follows that $u=z^{\prime}$ and so $[a, v]=z^{\prime}$ and $Q=\langle a, v\rangle \cong \mathrm{Q}_{8}$ which is normalized but not centralized by $H$ and $Q \cap H=\{1\}$. The structure of $G$ is uniquely determined.

Set $C=\left\langle h_{0}, h_{1} a\right\rangle$. Since $h_{0}^{2}=z,\left(h_{1} a\right)^{2}=z z^{\prime}$ and $\left[h_{0}, h_{1} a\right]=z$, we have that $C \cong \mathcal{H}_{2}$ and $C \cap Q=\left\langle z^{\prime}\right\rangle$, where $z^{\prime}$ is not a square in $C$. Also we have $[C, Q]=\{1\}$ and therefore we have obtained the group in part (f) of Theorem $\mathrm{B}$, which obviously satisfies the assumptions of that theorem, Our result is completely proved.

\section{Proof of Theorem C}

This theorem will be proved with a series of Propositions 1 to 12 .

Proposition 4.1. Let $G$ be a p-group with a cyclic intersection of any two distinct conjugate subgroups. Then each non-normal subgroup $X$ in $G$ possesses a cyclic subgroup of index $p$. 
Proof. Let $H$ be a maximal non-normal subgroup of $G$ containing $X$. Let $L>H$ be such that $|L: H|=p$ so that we have $L \unlhd G$. Since $H$ is not normal in $G$, there is $g \in G-L$ such that $H^{g} \neq H$. Hence we have $L=H H^{g}$ and $\left|H:\left(H \cap H^{g}\right)\right|=p$. By our assumption, $H \cap H^{g}$ is cyclic and so $H$ has a cyclic subgroup of index $p$. Since $X \leq H$, it follows that $X$ also has a cyclic subgroup of index $p$.

In the rest of the paper we assume:

(*) $\quad G$ is a $p$-group with cyclic intersection of any two distinct conjugate subgroups. Assume in addition that $G$ has a maximal non-normal subgroup $H$ which is neither cyclic nor abelian of type $(p, p)$ nor an ordinary quaternion group. We set $K=\mathrm{N}_{G}(H)$ so that $H<K<G$ and $K \unlhd G$ and let $L / H$ be a unique subgroup of order $p$ in $K / H$, where $L \unlhd G$. This notation will be fixed in the sequel.

Proposition 4.2. We have that $K / H \neq\{1\}$ is either cyclic or $p=2$ and $K / H \cong \mathrm{Q}_{8}$. Also we have $\Omega_{1}(K) \leq L$.

If $K$ does not possess a $G$-invariant subgroup isomorphic to $\mathrm{E}_{p^{2}}$, then $G$ is a 2-group of maximal class and order $\geq 2^{5}$ and if $|G|=2^{5}$, then $G \cong \mathrm{D}_{32}$ or $\mathrm{SD}_{32}$ and all these groups satisfy our assumption $(*)$.

From now on we always assume that $K$ has a G-invariant subgroup $U$ isomorphic to $\mathrm{E}_{p^{2}}$ and then we have $L=H U$ with $U_{0}=H \cap U \cong \mathrm{C}_{p}$ and $G / U$ is Dedekindian.

Proof. Suppose that $K / H$ has two distinct subgroups $K_{1} / H$ and $K_{2} / H$ of order $p$. Then $K_{1} \unlhd G, K_{2} \unlhd G$ and so $K_{1} \cap K_{2}=H \unlhd G$, a contradiction. Hence $L / H$ is a unique subgroup of order $p$ in $K / H$ and so $K / H$ is either cyclic or generalized quaternion. On the other hand, $K / H$ is Dedekindian and so $K / H \neq\{1\}$ is either cyclic or $p=2$ and $K / H \cong Q_{8}$. In any case, we have $\Omega_{1}(K) \leq L$.

Assume that $K$ does not have a $G$-invariant abelian subgroup of type $(p, p)$. By Lemma 1.1 in [1], we have $p=2$ and $K$ is a 2-group of maximal class and order $\geq 2^{4}$. In that case $K / H \cong$ Q $_{8}$ cannot happen and so $K / H$ is cyclic. It follows that $K^{\prime} \leq H$ and $K / K^{\prime} \cong \mathrm{E}_{4}$ and so $K=L$ and $K^{\prime}$ is a cyclic subgroup of index 2 in $H$ and $K^{\prime} \unlhd G$. Since $H$ has only two conjugates in $G$, we have $|G: K|=2$ and so $|G| \geq 2^{5}$. Since $H$ is not normal in $G$, we have $G^{\prime}>K^{\prime}$ and so $\left|G: G^{\prime}\right|=4$. By a well known result of O. Taussky, $G$ is a 2-group of maximal class and order $\geq 2^{5}$. However, $\mathrm{Q}_{32}$ does not satisfy (*) and so if $|G|=2^{5}$, then $G \cong \mathrm{D}_{32}$ or $\mathrm{SD}_{32}$.

Conversely, let $G$ be a 2 -group of maximal class and order $\geq 2^{5}$. Let $Z$ be a unique cyclic subgroup of index 2 in $G$. Let $H$ be any non-normal subgroup in $G$ so that we have $H \not \leq Z$ and set $H_{0}=H \cap Z \unlhd G$ with $\left|H: H_{0}\right|=2$. Hence if $g \in G$ is such that $H^{g} \neq H$, then we have $H \cap H^{g}=H_{0}$ is cyclic.

In the sequel we shall always assume that $K$ possesses a $G$-invariant abelian subgroup $U$ of type $(p, p)$. Since $\Omega_{1}(K) \leq L$, we have $U \leq L$. On 
the other hand, $G / U$ is Dedekindian and so $U \not \subset H$. We get $L=H U$ with $U_{0}=H \cap U \cong \mathrm{C}_{p}$.

Proposition 4.3. Assuming that $G$ is not a 2-group of maximal class, then it follows that $|G: K|=p$ and we may choose a $G$-invariant abelian subgroup $U$ of type $(p, p)$ in $L$ so that $\mathrm{C}_{p} \cong U_{0}=H \cap U \leq \mathrm{Z}(G)$. Also, $G^{\prime}$ covers $U / U_{0}$ and we have one of the following possibilities.

(a) We have

$$
\begin{gathered}
p=2, H \cong \mathrm{D}_{8}, \mathrm{Z}(L)=U \leq G^{\prime} \text { and } K=H * \mathrm{C}_{G}(H) \text { with } \\
U \leq \mathrm{C}_{G}(H) \text { and } H \cap \mathrm{C}_{G}(H)=U_{0} .
\end{gathered}
$$

Also, the unique cyclic subgroup of order 4 in $H$ is normal in $G$.

(b) We have $H \cong \mathrm{M}_{p^{n}}, n \geq 3$, (if $p=2$, then $n \geq 4$ ) or $H$ is abelian of type $\left(p^{s}, p\right), s \geq 2$. Set $H_{0}=\Omega_{1}(H)$ and then $H_{0} \cong \mathrm{E}_{p^{2}}, \mathrm{~N}_{G}\left(H_{0}\right)=K$ and $K / H_{0}$ is Dedekindian. There are two subcases:

(b1) If $S=H_{0} U$ is abelian, then $S \unlhd G$ is elementary abelian of order $p^{3}$ and either $H \cong \mathrm{M}_{p^{n}}, n \geq 3$, (if $p=2$. then $n \geq 4$ ) and in this case we have $U=\Omega_{1}(\mathrm{Z}(L)), L^{\prime}=U_{0}$, and $U \leq G^{\prime}$, or $H$ is abelian of type $\left(p^{s}, p\right), s \geq 2$, and in this case $L$ is abelian of type $\left(p^{s}, p, p\right)$ with $\mho_{1}(L)=\mho_{1}(H) \geq U_{0}$.

(b2) If $S=H_{0} U$ is nonabelian, then $p>2, S \cong \mathrm{S}\left(p^{3}\right) \unlhd G$ (the nonabelian group of order $p^{3}$ and exponent $p$ ) with $\mathrm{Z}(S)=U_{0}$. We have

$G=(Z * S)\langle e\rangle$, where $\mathrm{C}_{p^{m}} \cong Z=\mathrm{C}_{G}(S) \unlhd G, m \geq 2, S \cong \mathrm{S}\left(p^{3}\right) \unlhd G$,

$Z \cap S=\mathrm{Z}(S)=U_{0}, Z\langle e\rangle=\langle e\rangle \cong \mathrm{C}_{p^{m+1}}$ or $o(e)=p$ and $Z\langle e\rangle$

is either abelian of type $\left(p^{m}, p\right)$ or $Z\langle e\rangle \cong \mathrm{M}_{p^{m+1}}$, where in any case $e$ induces on $S$ an outer automorphism of order $p$ (normalizing $U$ and fusing the other $p$ maximal subgroups of $S$ ). We have $\mathrm{E}_{p^{2}} \cong G^{\prime}=U<S$ and $G$ is a group of class 3 . We have $\Omega_{1}(Z * S)=S$ and if $Z\langle e\rangle=\langle e\rangle \cong \mathrm{C}_{p^{m+1}}$, then $\Omega_{1}(G)=S$.

Conversely, groups $G$ defined in (b2) satisfy our assumption $(*)$.

Proof. By Proposition 1, $H$ possesses a cyclic subgroup of index $p$.

(i) First assume that $H$ is a 2-group of maximal class. In that case $U_{0}=U \cap H=\mathrm{Z}(H)$. If $|H|>2^{3}$, then we have $H / U_{0} \cong L / U \cong \mathrm{D}_{2^{n}}, n \geq 3$, contrary to the fact that $G / U$ is Dedekindian. It follows that $H \cong \mathrm{D}_{8}$ and because $|L / U|=4$, we get $L^{\prime} \leq H \cap U=U_{0}$ and so $L^{\prime}=U_{0} \leq \mathrm{Z}(G)$. Then we have $L=H * Z$, where $Z=\mathrm{C}_{L}(H), Z \cap H=U_{0}$ and $Z \cong \mathrm{C}_{4}$ or $\mathrm{E}_{4}$.

Let $\langle h\rangle$ be a unique cyclic subgroup of order 4 in $H$ and let $x \in G-K$ so that $H^{x} \neq H$. Since $H \cap H^{x}$ is cyclic, we get $H \cap H^{x}=\langle h\rangle$ for all $x \in G-K$. This gives $\langle h\rangle \unlhd G$. But $L /\langle h\rangle \cong \mathrm{E}_{4}$ and so $L$ contains exactly two distinct conjugates of $H$ in $G$ and this implies $|G: K|=2$. Let $t$ be an involution in $H-\langle h\rangle$. Because $U\langle t\rangle \unlhd G$ and $H$ is not normal in $G$, we get for an 
$x \in G-K, t^{x} \notin H$ and therefore we have $t^{x}=t u$ with some $u \in U-U_{0}$. Hence $[t, x]=u \in G^{\prime}$, which implies that $G^{\prime}$ covers $U / U_{0}$ and so in this case $U \leq G^{\prime}$.

Assume for a moment that $Z \cong \mathrm{C}_{4}$. In this case it is well known that $L \cong \mathrm{D}_{8} * \mathrm{C}_{4}$ contains a unique subgroup $Q$ isomorphic to $\mathrm{Q}_{8}$ and so $Q \unlhd G$. For any cyclic subgroup $\langle v\rangle$ of order 4 in $Q$ we have $U_{0}<\langle v\rangle$ and $U\langle v\rangle \unlhd G$. But then

$$
\langle v\rangle=(U\langle v\rangle) \cap Q \unlhd G,
$$

and so $G$ induces on $Q$ only inner automorphisms of $Q$. We get $G=Q * C$, where $C=\mathrm{C}_{G}(Q)$ and $Q \cap C=U_{0}$. Since $Q$ does not centralize $U$, we have $U \not \subset C$ and so $U \cap C=U_{0}=Q^{\prime}$. On the other hand, we get

$$
G^{\prime}=Q^{\prime} C^{\prime}=U_{0} C^{\prime} \leq C,
$$

contrary to $U \leq G^{\prime}$. We have proved that $Z \cong \mathrm{E}_{4}$ and $Z=\mathrm{Z}(L) \unlhd G$.

Suppose that $U \neq Z$ so that $U \cap Z=U_{0}, S=U Z \cong \mathrm{E}_{8}$ and $S \unlhd G$. Acting with an element $x \in G-K$ on three subgroups of order 4 in $S$ which contain $U_{0} \leq \mathrm{Z}(G)$, we see that $Z \unlhd G, U \unlhd G$ and so also we have $\mathrm{E}_{4} \cong S \cap H \unlhd G$. But we know that a cyclic subgroup of order 4 in $H$ is normal in $G$ and so we get $H \unlhd G$, a contradiction. We have proved that $U=Z=\mathrm{Z}(L)$.

Let $t$ be any involution in $H$. Since $U\langle t\rangle \unlhd G$ and $H \unlhd K$, it follows that

$$
(U\langle t\rangle) \cap H=\left\langle t, U_{0}\right\rangle \unlhd K \text {. }
$$

Thus, each element in $K$ induces on $H$ only inner automorphisms of $H$. It follows

$$
K=H * \mathrm{C}_{G}(H) \text { with } U \leq \mathrm{C}_{G}(H)=\mathrm{C}_{K}(H) \text { and } H \cap \mathrm{C}_{G}(H)=U_{0} .
$$

(ii) Now suppose that $H \cong \mathrm{M}_{p^{n}}, n \geq 3$, (where in case $p=2$ we have $n \geq 4$ ) or $H$ is abelian of type $\left(p^{s}, p\right), s \geq 2$. Set $H_{0}=\Omega_{1}(H) \cong \mathrm{E}_{p^{2}}$ so that $H_{0} \unlhd K$. It follows that $\mathrm{N}_{G}\left(H_{0}\right)=K$ and $K / H_{0}$ is Dedekindian. Set $S=H_{0} U \unlhd G$. We have

$$
L / U \cong H / U_{0} \text {, where } H^{\prime} \leq U_{0} \leq \mathrm{Z}(H) \text {, and so } L^{\prime} \leq H \cap U=U_{0} .
$$

If $L$ is nonabelian, then $L^{\prime}=U_{0} \leq \mathrm{Z}(G)$. In that case we act with $G / K$ on $p+1$ subgroups of order $p^{2}$ in $S$ which contain $U_{0} \leq \mathrm{Z}(G)$, where $U$ is the only one of them which is normal in $G$ and all $p$ other ones are fused with $G / K$ and so we get $|G: K|=p$. Also, if $h_{0} \in H_{0}-U_{0}$ and $x \in G-K$, then $h_{0}^{x}=h_{0} u$ with $u \in U-U_{0}$. Hence $G^{\prime}$ covers $U / U_{0}$ and so we have in this case $U \leq G^{\prime}$.

Now assume that $L$ is abelian so that $L$ is of type $\left(p^{s}, p, p\right)$. If $U_{0} \leq \mathrm{Z}(G)$, then with the same arguments as above, we get $|G: K|=p$ and $G^{\prime}$ covers $U / U_{0}$. Now suppose that $U_{0} \not \leq \mathrm{Z}(G)$. Then there is a subgroup $U_{1}$ of order $p$ in $U$ such that $U=U_{0} \times U_{1}$ and $U_{1} \leq \mathrm{Z}(G)$. We have

$$
\mho_{1}(L)=\mho_{1}(H) \unlhd G \text { and } \mho_{1}(H) \neq\{1\} \text { is cyclic . }
$$


Let $H_{1}$ be the subgroup of order $p$ in $\mho_{1}(H)$ so that we get $H_{1} \leq \mathrm{Z}(G)$. Then we replace $U$ with

$$
\mathrm{E}_{p^{2}} \cong U^{*}=U_{1} \times H_{1} \leq \mathrm{Z}(G)
$$

where

$$
U_{0}^{*}=U^{*} \cap H=H_{1} \leq \mathrm{Z}(G)
$$

and set $S^{*}=H_{0} U^{*}$. Now, working with $U^{*}, U_{0}^{*} \leq \mathrm{Z}(G)$ and $S^{*}=H_{0} U^{*}$ (instead of $U, U_{0}$ and $S$ ), we get with the same arguments as above that $|G: K|=p$ and that $G^{\prime}$ covers $U^{*} / U_{0}^{*}$. We write again $U$ and $U_{0}$ instead of $U^{*}$ and $U_{0}^{*}$, respectively, so that we may always assume that $U_{0}=U \cap H \leq \mathrm{Z}(G)$.

(ii1) Assume that $S=H_{0} U$ is abelian so that $S \cong \mathrm{E}_{p^{3}}$ and $S \unlhd G$. Suppose in addition that $H \cong \mathrm{M}_{p^{n}}, n \geq 3$, (where in case $p=2$ we have $n \geq 4)$. Then we have $L^{\prime}=H^{\prime}=U_{0} \leq \mathrm{Z}(G)$ and $U \leq G^{\prime}$. Let $\langle a\rangle$ be a cyclic subgroup of index $p$ in $H$ so that $\langle a\rangle$ covers $H / H_{0}$ (and $L / S$ ) and $\langle a\rangle \cap H_{0}=U_{0}=\langle z\rangle$. Let $t \in H_{0}-U_{0}$ so that we may set $[a, t]=z$. Suppose, by way of contradiction, that $U \not \mathbf{Z}(L)$. In that case, $\left|L: \mathrm{C}_{L}(U)\right|=p$ and so $\mathrm{C}_{L}(U)=\left\langle a^{p}\right\rangle S$. We may choose an element $u \in U-U_{0}$ so that $[a, u]=z^{-1}$. Then we get $[a, u t]=z^{-1} z=1$ so that we have

$$
\mathrm{Z}(L)=\left\langle a^{p}\right\rangle \times\langle u t\rangle \text { and } \mathrm{E}_{p^{2}} \cong \Omega_{1}(\mathrm{Z}(L))=\langle u t, z\rangle \unlhd G .
$$

But we know that $\mathrm{C}_{p} \cong G / K$ acts transitively on $p$ maximal subgroups of $S$ which contain $U_{0} \leq \mathrm{Z}(G)$ and which are distinct from $U$. Since $\langle u t, z\rangle \neq U$, we have a contradiction. Thus we have proved that $U \leq \mathrm{Z}(L)$ and so $U=$ $\Omega_{1}(\mathrm{Z}(L))$

Now assume that $H$ is abelian of type $\left(p^{s}, p\right), s \geq 2$. Suppose, by way of contradiction, that $L$ is nonabelian. In that case we have $L^{\prime}=U_{0} \leq \mathrm{Z}(G)$ and $\mathrm{C}_{L}(H)=H$. By Lemma 1.1 in [1], we get

$$
|L|=p|\mathrm{Z}(L)|\left|L^{\prime}\right| \text { and so }|L: \mathrm{Z}(L)|=p^{2} \text {. }
$$

Since $\mathrm{Z}(L)<H$, it follows that $\mathrm{Z}(L)$ is a maximal subgroup of $H$. If $\mathrm{Z}(L) \geq$ $H_{0}$, then $H_{0}=\Omega_{1}(\mathrm{Z}(L))$, which implies that $H_{0} \unlhd G$, a contradiction. It follows that $\mathrm{Z}(L)$ is a cyclic subgroup of index $p$ in $H$ and so $\mathrm{Z}(L)$ covers $H / H_{0}$ and $L / S$. Hence we get that $L=\mathrm{Z}(L) S$ is abelian, a contradiction. We have proved that $L$ is abelian of type $\left(p^{s}, p, p\right)$. Then we get $\mho_{1}(L)=\mho_{1}(H)$ and $\mho_{1}(H)$ is cyclic of order $\geq p$. Let $H_{1}$ be the subgroup of order $p$ in $\mho_{1}(H)$ so that $H_{1} \leq \mathrm{Z}(G)$ and $H_{1} \leq H_{0}$. If $H_{1} \neq U_{0}$, then $H_{0}=H_{1} \times U_{0} \leq \mathrm{Z}(G)$, contrary to $\mathrm{N}_{G}\left(H_{0}\right)=K$. Hence we have $H_{1}=U_{0}$ and so $\mho_{1}(L)=\mho_{1}(H) \geq$ $U_{0}$.

(ii2) Assume that $S=H_{0} U$ is nonabelian. If $p=2$, then $S \cong \mathrm{D}_{8}$. But $U$ and $H_{0}$ are the only two four-subgroups in $S$ and since $U \unlhd G$, it follows that $H_{0} \unlhd G$, a contradiction. Hence we have $p>2$ and $S \cong \mathrm{S}\left(p^{3}\right)$ (the nonabelian group of order $p^{3}$ and exponent $p$ ) with $S^{\prime}=\mathrm{Z}(S)=U_{0}$. We 
know that $U \leq G^{\prime}$. On the other hand, $G / U$ is Dedekindian and so abelian which implies that $G^{\prime} \leq U$ and therefore we have $G^{\prime}=U<S \unlhd G$. Since $U=G^{\prime} \not \leq \mathrm{Z}(S)$, it follows that $G$ is of class 3. Also, $U$ is a unique normal abelian subgroup of type $(p, p)$ in $G$. Indeed, if $V \cong \mathrm{E}_{p^{2}}, V \unlhd G$ and $V \neq U$, then the fact that $G / V$ is abelian Dedekindian implies that $G^{\prime} \leq V \cap U<U$, a contradiction. Set $Z=\mathrm{C}_{G}(S)$ so that $Z \unlhd G$ and $Z \cap S=U_{0}$. We know that $Z$ does not have a $G$-invariant abelian subgroup of type $(p, p)$ and so Lemma 1.4 in [1] implies that $Z \cong \mathrm{C}_{p^{m}}, m \geq 1$, is cyclic and so $\Omega_{1}(Z * S)=S$. If $Z * S=G$, then $G^{\prime}=U_{0} \cong \mathrm{C}_{p}$, a contradiction. Hence we have $Z * S<G$. On the other hand, a Sylow $p$-subgroup of $\operatorname{Aut}(S)$ is isomorphic to $\mathrm{S}\left(p^{3}\right)$ and so $G / Z \cong \mathrm{S}\left(p^{3}\right)$ and $|G:(Z * S)|=p$. We know that $|G| \geq p^{5}$ because $|H| \geq p^{3}$ and so $L=H U(<G)$ is of order $\geq p^{4}$. This implies that we have $m \geq 2$. Let $e$ be an element in $G-(Z * S)$ so that $e$ fixes $U$ and fuses the other $p$ maximal subgroups of $S$. Since $G / Z \cong \mathrm{S}\left(p^{3}\right)$ is of exponent $p$, we have $e^{p} \in Z$. If $Z\langle e\rangle$ is cyclic, then we have

$$
Z\langle e\rangle=\langle e\rangle \cong \mathrm{C}_{p^{m+1}} .
$$

In this case, $G / S$ is cyclic of order $\geq p^{2}$ and $\Omega_{1}(Z * S)=S$ together with $|Z| \geq p^{2}$ implies $\Omega_{1}(G)=S$. If $Z\langle e\rangle$ is noncyclic, then $Z\langle e\rangle$ splits over $Z$ and we may assume that $o(e)=p$. In this case $Z\langle e\rangle$ is either abelian of type $\left(p^{m}, p\right)$ or $Z\langle e\rangle \cong \mathrm{M}_{p^{m+1}}$. We have obtained the groups stated in part (b2) of our proposition.

It remains to be proved that these groups $G$ satisfy our condition (*). Let $X$ be any noncyclic and non-normal subgroup of order $\geq p^{3}$ in $G$. First assume that $|X \cap S|=p^{2}$ so that we have $X \cap S=S_{i}$ for some $i \in\{1,2, \ldots, p\}$, where $\left\{S_{1}, S_{2}, \ldots, S_{p}\right\}$ is the set of maximal subgroups of $S$ distinct from $U$ which are acted upon transitively by $G /(Z * S)$. Since $\Omega_{1}(Z * S)=S$, we have $\Omega_{1}(X \cap Z * S)=S_{i}$ and this implies that $X \leq Z * S$. Since $X \geq S_{i}>$ $U_{0}=(Z * S)^{\prime}$, it follows that $\mathrm{N}_{G}(X)=\mathrm{N}_{G}\left(S_{i}\right)=Z * S$ and then for each $g \in G-(Z * S)$, the intersection $X \cap X^{g}$ is cyclic.

Now assume that $|X \cap S|=p$. (If $|X \cap S|=1$, then $X \cap(Z * S)=\{1\}$ and then $|X| \leq p$, a contradiction.) In this case, $X_{0}=X \cap(Z * S)$ is cyclic of order $\geq p^{2}, X \not \leq Z * S$ and so $\left|X: X_{0}\right|=p$. On the other hand, $\mho_{1}(Z * S)=\mho_{1}(Z) \geq U_{0}$ and so $X_{0} \geq U_{0}$. We get $\mathrm{N}_{G}\left(X_{0}\right) \geq\langle Z * S, X\rangle=G$. Hence for each $g \in G$ with $X^{g} \neq X$, we see that $X \cap X^{g}=X_{0}$ is cyclic.

Finally, $Z S_{i} \cong \mathrm{C}_{p^{m}} \times \mathrm{C}_{p}, m \geq 2$, is not normal in $G$ but $Z \unlhd G$ and so our condition $(*)$ is satisfied. Proposition 3 is completely proved.

Proposition 4.4. If $U \cong \mathrm{E}_{p^{2}}$ is a G-invariant subgroup contained in $K=\mathrm{N}_{G}(H)$ such that $U_{0}=H \cap U \leq \mathrm{Z}(G)$, then we have $G^{\prime} \leq U$. Hence $G^{\prime}$ is elementary abelian of order $\leq p^{2}$ and so $G$ is of class at most 3 .

Proof. Assume that $G / U$ is nonabelian so that we have $p=2$ and $G / U$ is Hamiltonian. Let $Q / U$ be any ordinary quaternion subgroup in $G / U$ and 
we set

$$
Q_{0} / U=(Q / U)^{\prime}=\mathrm{Z}(Q / U)=(G / U)^{\prime} .
$$

We have $\left|Q: \mathrm{C}_{Q}(U)\right| \leq 2$ and so $Q_{0}<\mathrm{C}_{Q}(U)$ and let $y \in \mathrm{C}_{Q}(U)-Q_{0}$ so that $y^{2} \in Q_{0}-U$. Hence $U\langle y\rangle$ is an abelian maximal subgroup in $Q$. By lemma 1.1 in [1], we have

$$
2^{5}=|Q|=2\left|Q^{\prime}\right||\mathrm{Z}(Q)| \text {, where } \mathrm{Z}(Q) \leq Q_{0} \text { and } Q_{0} \cong \mathrm{E}_{8} \text { or } Q_{0} \cong \mathrm{C}_{4} \times \mathrm{C}_{2} \text {. }
$$

If $Q^{\prime}=Q_{0}$, then $\left|Q: Q^{\prime}\right|=4$ and so by a result of O. Taussky, $Q$ is of maximal class and order $2^{5}$, contrary to $U \unlhd Q$. Thus, we have $Q^{\prime}<Q_{0}$ and $Q^{\prime}$ covers $Q_{0} / Q$.

(i) First suppose that $Q_{0} \cong \mathrm{E}_{8}$. We know that $G / Q_{0}$ is elementary abelian and so in this case $\exp (G)=4$. In particular, we must have (according to Proposition 3) $H \cong \mathrm{D}_{8}$ or $\mathrm{C}_{4} \times \mathrm{C}_{2}$. Consider again an abelian maximal subgroup $U \times\langle y\rangle$ of $Q$, where $\langle y\rangle \cong \mathrm{C}_{4}$ and $y^{2} \in Q_{0}-U$. Since $U \times\langle y\rangle \unlhd G$, we get $y^{2} \in \mathrm{Z}(G)$. Hence $y^{2}$ is an involution in $K$ and since $\Omega_{1}(K) \leq L$ (see Propositions 2 and 3), we get $Q_{0}=\left\langle y^{2}\right\rangle \times U \leq L$. Set $H_{0}=Q_{0} \cap H \cong \mathrm{E}_{4}$, where $H_{0}>U_{0}$ and $\mathrm{N}_{G}\left(H_{0}\right)=K$. Now act with $G / K$ on three subgroups of order 4 in $Q_{0}$ which contain $U_{0} \leq \mathrm{Z}(G)$. We see that only $U$ is normal in $G$ and $H_{0} \neq H_{0}^{g}$ with some $g \in G-K$. But $y^{2} \in Q_{0}-U$ and $y^{2} \in \mathrm{Z}(G)$ and so $\left\langle y^{2}, U_{0}\right\rangle \unlhd G$, a contradiction.

(ii) We have proved that $Q_{0} \cong \mathrm{C}_{4} \times \mathrm{C}_{2}$ so that all elements in $Q_{0}-U$ are of order 4 and all elements in $Q-Q_{0}$ are of order 8. Since $Q^{\prime}$ covers $Q_{0} / U$ and $Q^{\prime}<Q_{0}$, we get $Q^{\prime} \cong \mathrm{C}_{4}$. On the other hand, $\Omega_{2}(Q)=Q_{0} \cong \mathrm{C}_{4} \times \mathrm{C}_{2}$ and so Lemma 42.1 in [1] implies that $Q$ can be defined with:

$$
Q=\left\langle a, b \mid a^{8}=b^{8}=1, a^{4}=b^{4}=z, a^{b}=a^{-1}\right\rangle,
$$

where

$$
\begin{gathered}
Q^{\prime}=\left\langle a^{2}\right\rangle \cong \mathrm{C}_{4}, \mathrm{Z}(Q)=\left\langle b^{2}\right\rangle \cong \mathrm{C}_{4}, \Omega_{2}(Q)=\left\langle a^{2}, b^{2}\right\rangle=Q_{0} \cong \mathrm{C}_{4} \times \mathrm{C}_{2}, \\
\Omega_{1}(Q)=U=\left\langle z, a^{2} b^{2}\right\rangle \cong \mathrm{E}_{4}, U_{0}=\langle z\rangle,
\end{gathered}
$$

and $A=\left\langle a, b^{2}\right\rangle \cong \mathrm{C}_{8} \times \mathrm{C}_{2}$ is a unique abelian maximal subgroup of $Q$. Also, it is easy to see that $\langle a\rangle$ is a characteristic subgroup in $Q$. Indeed, if $\theta \in \operatorname{Aut}(Q)$, then $A^{\theta}=A$ and so $b^{\theta} \in Q-A$. Suppose that $\langle a\rangle^{\theta} \neq\langle a\rangle$. Then we have $\langle a\rangle^{\theta}=\left\langle a b^{2}\right\rangle$ and we get

$$
\left(a b^{2}\right)^{b^{\theta}}=a^{-1} b^{-2}=a^{b^{\theta}}\left(b^{2}\right)^{b^{\theta}}=a^{-1} b^{2}
$$

and so we get $b^{4}=1$, a contradiction.

(iii) We know from Proposition 3 that $G^{\prime}$ covers $U / U_{0}$ and since $G / Q_{0}$ is elementary abelian (and so $\exp (G)=8$ ), we have $G^{\prime} \leq Q_{0}$. But $Q^{\prime}=\left\langle a^{2}\right\rangle$ with $\left\langle a^{4}\right\rangle=\langle z\rangle=U_{0}$ and so we get $G^{\prime}=Q_{0}$. In particular, we have $G>Q$ and $|G| \geq 2^{6}$. 
Since $\mathrm{C}_{Q}(U)=A=\left\langle a, b^{2}\right\rangle$ and $|Q: A|=2$, we see that $C=\mathrm{C}_{G}(U)$ covers $G / Q$, where $C \cap Q=A$ and $C>A$. On the other hand, $C / U$ does not possess an ordinary quaternion subgroup and so $C / U$ is abelian and therefore $C$ is of class $\leq 2$ with $C^{\prime} \leq U \leq \mathrm{Z}(C)$. Indeed, if $Q_{1} / U \cong \mathrm{Q}_{8}$ and $Q_{1} \leq C$, then by (ii) (since $Q / U$ was an arbitrary ordinary quaternion subgroup in $G / U)$, we have $U \not Z \mathrm{Z}\left(Q_{1}\right)$ which is not the case. For any $x, y \in C$, we have $\left[x^{2}, y\right]=[x, y]^{2}=1$ and so we have $\mho_{1}(C) \leq \mathrm{Z}(C)$. Since $a \in C$ and $a^{2} \in Q_{0}-U$, it follows that $Q_{0} \leq \mathrm{Z}(C)$ and so $C=\mathrm{C}_{G}(U)=\mathrm{C}_{G}\left(Q_{0}\right)$. In particular, we get $\mathrm{C}_{G}\left(b^{2}\right) \geq\langle Q, C\rangle=G$ which shows that $b^{2} \in \mathrm{Z}(G)$.

(iv) Now we show that $\mathrm{C}_{G}(Q)=\mathrm{Z}(Q)=\left\langle b^{2}\right\rangle=\mathrm{Z}(G)$. Indeed, set $R=\mathrm{C}_{G}(Q)$, where $R \cap Q=\mathrm{Z}(Q)=\left\langle b^{2}\right\rangle \leq \mathrm{Z}(G)$ and $b^{4}=z$ with $\langle z\rangle=U_{0}$. First suppose that $R$ has a $G$-invariant four-subgroup $U_{1}$. If $\left.U_{1}\right\rangle\langle z\rangle$, then set $U_{1}=U^{*}$ and if $U_{1} \nsupseteq\langle z\rangle$, then considering $\mathrm{E}_{8} \cong U_{1} \times\langle z\rangle$, we may choose in $U_{1} \times\langle z\rangle$ a $G$-invariant four-subgroup $U^{*}$ such that $U^{*}>\langle z\rangle$ and we have in any case $U^{*} \cap U=\langle z\rangle=U^{*} \cap Q$. Since $U^{*} \cap H=\langle z\rangle=U_{0} \leq \mathrm{Z}(G)$ and $\left|\left(H U^{*}\right): H\right|=2$, we have $H U^{*} \leq K=\mathrm{N}_{G}(H)$ and so $L=H U^{*}$. By Proposition 3 (using $U^{*}$ instead of $U$ ), we get that $G^{\prime}$ covers $U^{*} / U_{0}$, contrary to to the fact that $G^{\prime}=Q_{0}$. Hence $R$ does not have a $G$-invariant four-subgroup. By Lemma 1.4 in [1], $R$ is either cyclic or $R$ is of maximal class. But $\left\langle b^{2}\right\rangle \cong \mathrm{C}_{4}$ and $\left\langle b^{2}\right\rangle \leq \mathrm{Z}(R)$ and so $R$ must be cyclic. Assume that $R>\left\langle b^{2}\right\rangle$ which together with $\exp (G)=8$ gives $R \cong \mathrm{C}_{8}$. We may choose a generator $r$ of $R$ so that $r^{2}=b^{-2}$ and then $i=r b$ is an involution in $G-Q$ since $i^{2}=(r b)^{2}=r^{2} b^{2}=b^{-2} b^{2}=1$. We have

$$
a^{i}=a^{r b}=a^{b}=a^{-1} \text { and so }[a, i]=a^{-2} \notin U,
$$

contrary to the fact that $G / U$ is Hamiltonian, where for each $x \in G$ with $x^{2} \in U$ we must have $[G, x] \leq U$.

(v) We study the automorphisms of $Q$ induced on $Q$ by elements of $C$, where $C \cap Q=A$. Now, $A$ induces on $Q$ the inner automorphisms given by:

$$
b^{a}=a^{-1} b a=b\left(b^{-1} a^{-1} b\right) a=b a^{2}, b^{a^{2}}=\left(b a^{2}\right)^{a}=b a^{4}=b z .
$$

Let $x \in C-A$ so that $x$ centralizes $Q_{0}=\left\langle a^{2}, b^{2}\right\rangle$ and $x$ normalizes $\langle a\rangle$ (because $\langle a\rangle$ is characteristic in $Q$ ) which gives $a^{x}=a z^{\epsilon}$, where $\epsilon \in\{0,1\}$. Note that $b^{x}=b y$ with some $y \in A=\left\langle a, b^{2}\right\rangle$. But $x$ normalizes (centralizes ) $Q_{0}=\left\langle a^{2}, b^{2}\right\rangle \cong \mathrm{C}_{4} \times \mathrm{C}_{2}$ and so $x$ must also normalize $\left\langle a^{2}, b\right\rangle \cong \mathrm{M}_{16}$ and so $y \in\left\langle a^{2}, b^{2}\right\rangle$. Then we get (noting that $b^{2} \in \mathrm{Z}(G)$ ):

$$
b^{2}=\left(b^{2}\right)^{x}=\left(b^{x}\right)^{2}=(b y)^{2}=b y b y=b^{2}\left(b^{-1} y b\right) y=b^{2} y^{b} y,
$$

and so we have $y^{b}=y^{-1}$ and this implies $y \in\left\langle a^{2}\right\rangle$.

(vi) We have proved that each element $x \in C-A$ induces on $Q$ an automorphism given by:

$$
b^{x}=b y, \text { where } y \in\left\langle a^{2}\right\rangle \text { and } a^{x}=a z .
$$


Indeed, if $\epsilon=0$, i.e., $a^{x}=a$, then $x$ would induce on $Q$ an inner automorphism, contrary to $\mathrm{C}_{G}(Q)=\mathrm{Z}(Q)$. Since $b^{x^{2}}=b y^{2}$ and $a^{x^{2}}=a$, we have $x^{2} \in Q$. Setting $G_{0}=\langle x\rangle Q$, where $\left|G_{0}: Q\right|=2$, we see that $G_{0}=G$ and so $G^{\prime}=Q^{\prime}=\left\langle a^{2}\right\rangle \cong \mathrm{C}_{4}$ because

$$
[b, x]=y \in\left\langle a^{2}\right\rangle \text { and }[a, x]=z=a^{4}
$$

and so $G /\left\langle a^{2}\right\rangle$ is abelian. On the other hand, we know that $G^{\prime}=Q_{0}$. This is a final contradiction and our proposition is proved.

Proposition 4.5. Suppose that we have the case (a) of Proposition 3, where $H \cong \mathrm{D}_{8}$. Then $K / H$ is cyclic and we have the following possibilities:

(a)

$$
G=(\langle a\rangle \times\langle b\rangle)\langle i\rangle, \text { where }\langle a\rangle \cong\langle b\rangle \cong \mathrm{C}_{4}
$$

and $i$ is an involution with $a^{i}=a^{-1}$ and $b^{i}=b^{-1}$ or $b^{i}=b a^{2} b^{2}$.

(b) $G$ is a unique group of order $2^{5}$ and class 3 with $\Omega_{2}(G) \cong \mathrm{C}_{2} \times \mathrm{D}_{8}$ which is defined in Theorem 52.2(a) in [2] for $n=2$.

(c)

$$
G=(\langle h\rangle \times\langle g\rangle)\langle i\rangle, \text { where }\langle h\rangle \cong \mathrm{C}_{4},\langle g\rangle \cong \mathrm{C}_{2^{m}}, m \geq 3,
$$

and $i$ is an involution with $h^{i}=h^{-1}$ and $g^{i}=g^{1+2^{m-1}}$. Here we have $|G|=2^{m+3}, G^{\prime}=\left\langle h^{2}, g^{2^{m-1}}\right\rangle \cong \mathrm{E}_{4}, G^{\prime} \leq \mathrm{Z}(G), \mathrm{Z}(G)=\left\langle h^{2}\right\rangle \times\left\langle g^{2}\right\rangle \cong$ $\mathrm{C}_{2} \times \mathrm{C}_{2^{m-1}}$. Finally, $\langle h, i\rangle \cong \mathrm{D}_{8}$ and $\langle g, i\rangle \cong \mathrm{M}_{2^{m+1}}$ are not normal in $G$.

(d) $G$ is a special group of order $2^{6}$ given with:

$G=(H \times\langle a\rangle)\langle g\rangle$, where $H=\left\langle h, i \mid h^{4}=i^{2}=1, h^{i}=h^{-1}, h^{2}=z\right\rangle \cong \mathrm{D}_{8}$, $\langle a\rangle \cong \mathrm{C}_{4}, a^{2}=z^{\prime}, g^{2}=z z^{\prime},[g, h]=1,[g, i]=[g, a]=z^{\prime}$.

We have $G^{\prime}=\left\langle z, z^{\prime}\right\rangle \cong \mathrm{E}_{4},\langle h, i\rangle \cong \mathrm{D}_{8}$ is not normal in $G$ but $\langle h\rangle \unlhd G$, and $\langle i, a\rangle \cong \mathrm{C}_{2} \times \mathrm{C}_{4}$ is not normal in $G$ but $\langle a\rangle \unlhd G$.

Conversely, all the above groups satisfy our assumption $(*)$.

Proof. By Proposition 4, we have $G^{\prime}=U \cong \mathrm{E}_{4}$.

(i) First assume $K / H \cong \mathrm{Q}_{8}$ so that we have $|G|=2^{7}$. We set $C=$ $\mathrm{C}_{G}(H)=\mathrm{C}_{K}(H)$ so that we have $K=H * C$ with $U \leq C, H \cap C=U_{0}$ and $C / U_{0} \cong Q_{8}$. Let $C_{1} / U_{0}$ and $C_{2} / U_{0}$ be two distinct cyclic subgroups of order 4 in $C / U_{0}$ so that $C_{1}$ and $C_{2}$ are abelian and $C_{1} \cap C_{2}=U$. It follows that $U \leq \mathrm{Z}(C)$ and so we get $U=\mathrm{Z}(K)$ and $\left|C^{\prime}\right|=2$ and therefore we have $U=U_{0} \times C^{\prime}$, where we set $U_{0}=\langle z\rangle$ and $C^{\prime}=\left\langle z^{\prime}\right\rangle$. Also we have $C=\mathrm{C}_{G}(L)$ and $C \unlhd G, C^{\prime} \unlhd G$, which implies $U \leq \mathrm{Z}(G)$. Thus we get $U=\mathrm{Z}(G)=G^{\prime}$ and for any $x, y \in G$ we have $\left[x^{2}, y\right]=[x, y]^{2}=1$ and therefore $\mho_{1}(G) \leq \mathrm{Z}(G)$ and so $U=\Phi(G)$, which shows that $G$ is special. Set $H=\left\langle h, t \mid h^{4}=t^{2}=1, h^{t}=h^{-1}\right\rangle \cong \mathrm{D}_{8}$ and we have $\langle h\rangle \unlhd G$ (Proposition $3(\mathrm{a}))$. 
(i1) Suppose that $C$ splits over $U_{0}$ and so we have in this case $C=\langle z\rangle \times C_{0}$, where $C_{0}=\left\langle c_{1}, c_{2}\right\rangle \cong \mathrm{Q}_{8}$ and $C_{0}^{\prime}=\left\langle z^{\prime}\right\rangle$. Since $\langle t\rangle \times C_{0}$ has no cyclic subgroup of index 2, Proposition 1 implies that $\langle t\rangle \times C_{0} \unlhd G$. But then we have

$$
C_{0}=C \cap\left(\langle t\rangle \times C_{0}\right) \unlhd G
$$

and each element in $G$ induces on $C_{0}$ an inner automorphism (otherwise, a cyclic subgroup of order 4 in $C_{0}$ would be contained in $G^{\prime}$, contrary to Proposition 4). This implies

$$
G=C_{0} * G_{0},
$$

where

$$
G_{0}=\mathrm{C}_{G}\left(C_{0}\right), C_{0} \cap G_{0}=\left\langle z^{\prime}\right\rangle=\mathrm{Z}\left(C_{0}\right), G_{0} \cap K=L, K=H \times C_{0},
$$

and $G_{0}$ is special of order $2^{5}$ with $\mathrm{Z}\left(G_{0}\right)=U$. Since $\langle h\rangle \unlhd G$ and $h^{t}=h^{-1}$, there is $g \in G_{0}-L$ such that $[g, h]=1$. But $\langle t\rangle U \unlhd G$ and $H$ is not normal in $G$, and so we get $t^{g}=t u$ with $u \in\left\{z^{\prime}, z z^{\prime}\right\}$. However, if $t^{g}=t z z^{\prime}$, then we replace $g$ with $g^{\prime}=g h$ (noting that $g^{\prime} \in G_{0}-L$ and $g^{\prime}$ also centralizes $h$ ) and get

$$
t^{g^{\prime}}=\left(t z z^{\prime}\right)^{h}=(t z) z z^{\prime}=t z^{\prime} .
$$

Hence writing again $g$ instead of $g^{\prime}$, we may assume from the start that $t^{g}=t z^{\prime}$ and so $[t, g]=z^{\prime}$. We have $g^{2} \in U$ and so we have $g^{2} \in\left\{1, z^{\prime}, z z^{\prime}, z\right\}$.

If $g^{2}=1$, then $[g, t]=z^{\prime}$ gives that $\langle g, t\rangle \cong \mathrm{D}_{8}$ with $\langle g, t\rangle^{\prime}=\left\langle z^{\prime}\right\rangle$, where the unique cyclic subgroup $\langle g t\rangle$ of order 4 in $\langle g, t\rangle$ must be normal in $G$. Indeed, if $\langle g, t\rangle \unlhd G$, then $\langle g t\rangle \unlhd G$, and if $\langle g, t\rangle$ is not normal in $G$, then Proposition 3(a) implies that $\langle g t\rangle \unlhd G$. However, $[g t, h]=z$ but $(g t)^{2}=[g, t]=z^{\prime} \neq z$ and so $\langle g t\rangle$ is not normal in $G$, a contradiction. This kind of argument we shall use here several times.

If $g^{2}=z^{\prime}$, then $c_{1}^{2}=z^{\prime}$ together with $\left[g, c_{1}\right]=1$ implies that $g c_{1}$ is an involution. In that case, $\left[t, g c_{1}\right]=z^{\prime}$ shows that $\left\langle t, g c_{1}\right\rangle \cong \mathrm{D}_{8}$ with $\left\langle t, g c_{1}\right\rangle^{\prime}=\left\langle z^{\prime}\right\rangle$. But then $\mathrm{C}_{4} \cong\left\langle t g c_{1}\right\rangle$ is not normal in $G$ since $\left[t g c_{1}, h\right]=z$, a contradiction.

If $g^{2}=z z^{\prime}$, then $(g h)^{2}=z^{\prime}=c_{1}^{2}$ together with $\left[g h, c_{1}\right]=1$ implies that $g h c_{1}$ is an involution. In that case, $\left[t, g h c_{1}\right]=z^{\prime} z$ shows that $\left\langle t, g h c_{1}\right\rangle \cong \mathrm{D}_{8}$ with $\left\langle t, g h c_{1}\right\rangle^{\prime}=\left\langle z^{\prime} z\right\rangle$. But then $\mathrm{C}_{4} \cong\left\langle t g h c_{1}\right\rangle$ is not normal in $G$ since $\left[t g h c_{1}, g\right]=z^{\prime}$, a contradiction.

If $g^{2}=z$, then $g h$ is an involution. In this case, $[t, g h]=z^{\prime} z$ shows that $\langle t, g h\rangle \cong \mathrm{D}_{8}$ with $\langle t, g h\rangle^{\prime}=\left\langle z^{\prime} z\right\rangle$. But then $\mathrm{C}_{4} \cong\langle t g h\rangle$ is not normal in $G$ since $[t g h, g]=z^{\prime}$, a contradiction.

(i2) We have proved that $C$ does not split over $U_{0}$. Since $C$ is twogenerator with $C^{\prime}=\left\langle z^{\prime}\right\rangle$, it follows that $C$ is minimal nonabelian. We have $\Omega_{1}(C)=U \cong \mathrm{E}_{4}$ and so $C$ is metacyclic. Hence we may choose generators $c_{1}, c_{2}$ of $C$ so that we have

$$
\mathcal{H}_{2} \cong C=\left\langle c_{1}, c_{2} \mid c_{1}^{4}=c_{2}^{4}=1, c_{1}^{c_{2}}=c_{1}^{-1}\right\rangle,
$$


where $c_{1}^{2}=z^{\prime}, c_{2}^{2}=z z^{\prime}, z$ is not a square in $C$.

Since $\langle h\rangle \unlhd G$ and $h^{t}=h^{-1}$, it follows that $\mathrm{C}_{G}(h)$ covers $G / K$. Let $g \in \mathrm{C}_{G}(h)-K$ so that $[h, g]=1$ and $g^{2} \in\left\langle z, z^{\prime}\right\rangle$. Because $\langle t\rangle U \unlhd G,\langle h\rangle \unlhd G$ and $H$ is not normal in $G$, it follows that $t^{g}=t u$ with $u \in U-U_{0}$. Replacing $g$ with $g h$, if necessary, we may assume from the start that $t^{g}=t z^{\prime}$ and so we have $[g, t]=z^{\prime}$.

If $g$ normalizes $\left\langle c_{1}\right\rangle$, then replacing $g$ with $g^{\prime}=g c_{2}$ (if necessary), we may assume that $g^{\prime}$ centralizes $\left\langle c_{1}\right\rangle$ (and we note that $g^{\prime}$ acts the same way on $H$ as $g$ does). In this case we write again $g$ instead of $g^{\prime}$ and we have $\left[g, c_{1}\right]=z^{\epsilon}$ with $\epsilon=0$. If $g$ does not normalize $\left\langle c_{1}\right\rangle$, then we have $\left[g, c_{1}\right]=z z^{\prime}$ or $\left[g, c_{1}\right]=z$. If in this case $\left[g, c_{1}\right]=z z^{\prime}$, then again replacing $g$ with $g^{\prime}=g c_{2}$, we get

$$
\left[g^{\prime}, c_{1}\right]=\left[g c_{2}, c_{1}\right]=\left(z z^{\prime}\right) z^{\prime}=z .
$$

Hence writing again $g$ instead of $g^{\prime}$, we may assume from the start that $\left[g, c_{1}\right]=z^{\epsilon}$ with $\epsilon=1$. Hence we have in any case $\left[g, c_{1}\right]=z^{\epsilon}$, where $\epsilon \in\{0,1\}$.

If $g^{2}=1$, then $[g, t]=z^{\prime}$ shows that $\langle g, t\rangle \cong \mathrm{D}_{8}$ with $\langle g, t\rangle^{\prime}=\left\langle z^{\prime}\right\rangle$. But then $\mathrm{C}_{4} \cong\langle g t\rangle$ is not normal in $G$ since $[g t, h]=z$, a contradiction.

Assume that $g^{2}=z^{\prime}$. If $\epsilon=0$, then we have $\left[g, c_{1}\right]=1$ and so $g c_{1}$ is an involution. Then $\left[t, g c_{1}\right]=z^{\prime}$ shows that $\left\langle t, g c_{1}\right\rangle \cong \mathrm{D}_{8}$ with $\left\langle t, g c_{1}\right\rangle^{\prime}=\left\langle z^{\prime}\right\rangle$. But then $\mathrm{C}_{4} \cong\left\langle t g c_{1}\right\rangle$ is not normal in $G$ since $\left[t g c_{1}, h\right]=z$, a contradiction. Thus we must have $\epsilon=1$ and so we get $\left[g, c_{1}\right]=z$. We compute

$$
\left(g h c_{1}\right)^{2}=z^{\prime} z \cdot z^{\prime} \cdot\left[c_{1}, g h\right]=z z=1,
$$

and so $g h c_{1}$ is an involution. Then $\left[t, g h c_{1}\right]=z^{\prime} z$ shows that $\left\langle t, g h c_{1}\right\rangle \cong \mathrm{D}_{8}$ with $\left\langle t, g h c_{1}\right\rangle^{\prime}=\left\langle z^{\prime} z\right\rangle$. But then $\mathrm{C}_{4} \cong\left\langle t g h c_{1}\right\rangle$ is not normal in $G$ since $\left[\operatorname{tgh} c_{1}, h\right]=z$, a contradiction.

If $g^{2}=z$, then $g h$ is an involution. Then $[t, g h]=z^{\prime} z$ shows that $\langle t, g h\rangle \cong$ $\mathrm{D}_{8}$ with $\langle t, g h\rangle^{\prime}=\left\langle z^{\prime} z\right\rangle$. But then $\mathrm{C}_{4} \cong\langle t g h\rangle$ is not normal in $G$ since $[\operatorname{tgh}, g]=z^{\prime}$, a contradiction.

Suppose that $g^{2}=z z^{\prime}$. Assume in addition that $\epsilon=0$ and so $\left[g, c_{1}\right]=1$. In this case we have

$$
\left(g h c_{1}\right)^{2}=z z^{\prime} \cdot z \cdot z^{\prime}=1
$$

and so $g h c_{1}$ is an involution. Then $\left[t, g h c_{1}\right]=z^{\prime} z$ shows that $\left\langle t, g h c_{1}\right\rangle \cong \mathrm{D}_{8}$ with $\left\langle t, g h c_{1}\right\rangle^{\prime}=\left\langle z^{\prime} z\right\rangle$. But then $\mathrm{C}_{4} \cong\left\langle t g h c_{1}\right\rangle$ is not normal in $G$ since $\left[t g h c_{1}, g\right]=z^{\prime}$, a contradiction. Hence we must have $\epsilon=1$ and so $\left[g, c_{1}\right]=z$. In this case, $g c_{1}$ is an involution since $\left(g c_{1}\right)^{2}=z z^{\prime} \cdot z^{\prime} \cdot z=1$. Then $\left[t, g c_{1}\right]=z^{\prime}$ shows that $\left\langle t, g c_{1}\right\rangle \cong \mathrm{D}_{8}$ with $\left\langle t, g c_{1}\right\rangle^{\prime}=\left\langle z^{\prime}\right\rangle$. But then $\mathrm{C}_{4} \cong\left\langle t g c_{1}\right\rangle$ is not normal in $G$ since $\left[t g c_{1}, g\right]=z^{\prime} z$, a contradiction. We have finally proved that here $K / H \cong \mathrm{Q}_{8}$ is not possible. 
(ii) Now assume that $K / H \neq\{1\}$ is cyclic. Here we have $K=H \times\langle a\rangle$ with $o(a)=2^{n}, n \geq 1$, where we set

$$
\begin{gathered}
\Omega_{1}(\langle a\rangle)=\left\langle z^{\prime}\right\rangle, U_{0}=\langle z\rangle=\mathrm{Z}(H), \\
\left\langle h, h^{\prime} \mid h^{4}=\left(h^{\prime}\right)^{2}=1,\left[h, h^{\prime}\right]=z, z^{2}=1\right\rangle \cong \mathrm{D}_{8}, U=\left\langle z, z^{\prime}\right\rangle=G^{\prime} .
\end{gathered}
$$

Since $\langle h\rangle \unlhd G$ (Proposition $3(\mathrm{a}))$ and $h^{h^{\prime}}=h^{-1}$, it follows that $\mathrm{C}_{G}(h)$ covers $G / H \cong \mathrm{C}_{2}$. Let $g \in \mathrm{C}_{G}(h)-K$ so that we have $\left(h^{\prime}\right)^{g}=h^{\prime} u$ for some $u \in U-U_{0}$ (noting that $\langle h\rangle \unlhd G$ and $\left\langle U\left\langle h^{\prime}\right\rangle\right\rangle \unlhd G$ but $H$ is not normal in $G$ ) and so replacing $g$ with $g h$ (if necessary), we may assume from the start that $\left(h^{\prime}\right)^{g}=h^{\prime} z^{\prime}$ and so we have $\left[g, h^{\prime}\right]=z^{\prime}$.

(ii1) Assume that $K=L$ and $z^{\prime} \in \mathrm{Z}(G)$. In this case we have $\mathrm{Z}(K)=$ $\mathrm{Z}(L)=U=\mathrm{Z}(G)$ and $\mho_{1}(G) \leq \mathrm{Z}(G)$. Hence $G$ is a special group of order $2^{5}$. In particular, all elements in $G-K$ are of order $\leq 4$. Suppose that there is an involution $t \in \mathrm{C}_{G}(h)-K$. Then we have $\left[h^{\prime}, t\right]=u \in U-\langle z\rangle$ and therefore $\left\langle h^{\prime}, t\right\rangle \cong \mathrm{D}_{8}$ with $\left\langle h^{\prime}, t\right\rangle^{\prime}=\langle u\rangle$. Then we must have $\mathrm{C}_{4} \cong\left\langle h^{\prime} t\right\rangle \unlhd G$. On the other hand, $\left[h^{\prime} t, h\right]=z$, a contradiction. Hence there is no involution in $\mathrm{C}_{G}(h)-K$. If $g^{2}=z$, then $h g$ is an involution in $\mathrm{C}_{G}(h)-K$, a contradiction. Hence we have

$$
g^{2} \in\left\{z^{\prime}, z z^{\prime}\right\} \text { and }\langle h, g\rangle=\langle h\rangle \times\langle g\rangle \cong \mathrm{C}_{4} \times \mathrm{C}_{4} .
$$

We set $h^{\prime}=i$ so that $G=(\langle h\rangle \times\langle g\rangle)\langle i\rangle$ with $h^{i}=h^{-1}$ and $g^{i}=g z^{\prime}$. We have obtained two groups of order $2^{5}$ stated in part (a) of our proposition, which obviously satisfy our assumption $(*)$.

(ii2) Assume that $K=L$ and $z^{\prime} \notin \mathrm{Z}(G)$. Then we have $\left[g, z^{\prime}\right]=z$. Suppose that there is an element $y \in G-K$ of order $\leq 4$. We claim that in this case we have $y^{2} \in U$. Indeed, if $y^{2}$ is a noncentral involution in $K=L$, then $y^{2}$ inverts $\langle h\rangle$ and $y$ normalizes $\langle h\rangle$ (since $\langle h\rangle \unlhd G$ ), a contradiction. Hence we have $y^{2} \in U$ and so $y^{2} \in\langle z\rangle$ since $\left[y, z^{\prime}\right]=z$. We get $D=\langle y, U\rangle \cong \mathrm{D}_{8}$ and $D \unlhd G$ with $\mathrm{Z}(D)=\langle z\rangle=D^{\prime}$. Since $G^{\prime}=U$ is elementary abelian, each element in $G$ induces an inner automorphism on $D$. Hence we have $G=D * C$, where $C=\mathrm{C}_{G}(D)$ and $D \cap C=\langle z\rangle$. Since $|C|=2^{3}$ and $z \in \mathrm{Z}(C)$, we have $C^{\prime} \leq\langle z\rangle$. This gives that $G^{\prime}=\langle z\rangle$, contrary to Proposition 3(a). We have proved that all elements in $G-K$ are of order 8 and so $\Omega_{2}(G) \cong \mathrm{C}_{2} \times \mathrm{D}_{8}$. Since $g$ centralizes $\langle h\rangle$, we must have $\left\langle g^{2}\right\rangle=\langle h\rangle$ and so we may assume that $g^{2}=h$. Indeed, if $\left\langle g^{2}\right\rangle=\left\langle h z^{\prime}\right\rangle$, then $g$ would centralize $h$ and $h z^{\prime}$ and so $g$ would centralize $z^{\prime}$, a contradiction. We have obtained a unique group $G$ of order $2^{5}$ and class 3 with $\Omega_{2}(G) \cong \mathrm{C}_{2} \times \mathrm{D}_{8}$ which is defined in Theorem 52.2 (a) in [2] for $n=2$ (stated in part (b) of our proposition). This group obviously satisfies our assumption $(*)$.

(ii3) Assume that $K>L$, i.e., $o(a)=2^{n}, n \geq 2$. Then there is an element $w \in\langle a\rangle$ of order 4 so that $w^{2}=z^{\prime}$. We have

$$
\langle z, w\rangle=\langle z\rangle \times\langle w\rangle \unlhd G \text { and so } \mho_{1}(\langle z\rangle \times\langle w\rangle)=\left\langle z^{\prime}\right\rangle \unlhd G,
$$


which implies that $G^{\prime}=U \leq \mathrm{Z}(G)$. We have also $\mho_{1}(G) \leq \mathrm{Z}(G)$. Since $G / L$ is abelian and $K / L \neq\{1\}$ is cyclic, we have here two subcases.

(ii3a) Suppose that $G / L$ is cyclic and so if $g \in \mathrm{C}_{G}(h)-K$, then $\langle g\rangle$ covers $G / L,\left[h^{\prime}, g\right]=z^{\prime}$ with $\left\langle z^{\prime}\right\rangle=\Omega_{1}\left(\left\langle g^{2}\right\rangle\right)$ and $o(g)=2^{m}, m \geq 3$. Hence we have $\left\langle g, h^{\prime}\right\rangle \cong \mathrm{M}_{2^{m+1}}$. Setting $h^{\prime}=i$, we get

$$
G=(\langle h\rangle \times\langle g\rangle)\langle i\rangle
$$

where

$$
\langle h\rangle \cong \mathrm{C}_{4},\langle g\rangle \cong \mathrm{C}_{2^{m}}, m \geq 3, h^{i}=h^{-1}, g^{i}=g^{1+2^{m-1}} .
$$

We have obtained the groups stated in part (c) of our proposition. Conversely, let $X$ be a non-normal and noncyclic subgroup of order $\geq 2^{3}$ in $G$. We see that $A=\langle h\rangle \times\langle g\rangle$ is an abelian maximal subgroup in $G$. If $X \cap A$ is noncyclic, then $X \cap A \geq\left\langle z, z^{\prime}\right\rangle=G^{\prime}$ and so $X \unlhd G$, a contradiction. Hence $X \cap A$ is cyclic and then $X \not \subset A$ so that $|X:(X \cap A)|=2$. It follows that $\mathrm{N}_{G}(X \cap A) \geq\langle A, X\rangle=G$ and so $X \cap A \unlhd G$. Thus, if $g \in G$ is such that $X^{g} \neq X$, then $X \cap X^{g}=X \cap A$ is cyclic. Finally, $\langle h, i\rangle \cong \mathrm{D}_{8}$ and $[i, g]=z^{\prime} \notin\langle h, i\rangle$ and so $\langle h, i\rangle$ is not normal in $G$. Hence our groups satisfy the assumption $(*)$.

(ii3b) $G / L$ is noncyclic abelian so that $G / L$ splits over $K / L$, where $K=$ $H \times\langle a\rangle$ with $o(a)=2^{n}, n \geq 2$, and $\Omega_{1}(\langle a\rangle)=\left\langle z^{\prime}\right\rangle$. We have $G=K G_{0}$, where $K \cap G_{0}=L$ and $\left|G_{0}: L\right|=2$. Since $G^{\prime}=U=\left\langle z, z^{\prime}\right\rangle \leq \mathrm{Z}(G)$ and $\left.\mho_{1}(G) \leq \mathrm{Z}(G)\right)$, we have that $G_{0}$ is one of two groups defined in part (a) of this proposition, where there is $g \in G_{0}-L$ such that $\langle g, h\rangle=\langle g\rangle \times\langle h\rangle$, $\left[h^{\prime}, g\right]=z^{\prime}$ and $g^{2}=z^{\epsilon} z^{\prime}$ with $\epsilon=0,1$.

Suppose that $\epsilon=0$ so that $g^{2}=z^{\prime}$ and so $h^{\prime}$ inverts each element in $\langle g, h\rangle$. Consider the subgroup $H_{1}=\left\langle h^{\prime}, g\right\rangle \cong \mathrm{D}_{8}$ with $\mathrm{Z}\left(\left\langle h^{\prime}, g\right\rangle\right)=\left\langle z^{\prime}\right\rangle$. If $H_{1} \unlhd G$, then $\langle g\rangle \unlhd G$ and if $H_{1}$ is not normal in $G$, then Proposition 3(a) shows that also $\langle g\rangle \unlhd G$. Hence in any case we have $\langle g\rangle \unlhd G$. Since $\langle a\rangle$ centralizes $h^{\prime}$, it follows that $\langle a\rangle \times\langle z\rangle$ normalizes $H_{1}$. On the other hand, $\left[h, h^{\prime}\right]=z$ and so $\langle h\rangle$ does not normalize $H_{1}$ a so we get

$$
\mathrm{N}_{G}\left(H_{1}\right)=H_{1}(\langle a\rangle \times\langle z\rangle) .
$$

If $w$ is an element of order 4 in $\langle a\rangle$, then we have $w^{2}=z^{\prime}$ and so $\left(H_{1}\langle w\rangle\right) / H_{1}$ and $\left(H_{1}\langle z\rangle\right) / H_{1}$ are two distinct subgroups of order 2 in $\mathrm{N}_{G}\left(H_{1}\right) / H_{1}$, contrary to Proposition 2. We have proved that we must have $\epsilon=1$ and so $g^{2}=z z^{\prime}$.

Assume that there is an element $w \in\langle a\rangle$ of order 4 such that $w^{2}=z^{\prime}$ and $[w, g]=1$. Then we have

$$
(w g)^{2}=w^{2} g^{2}=z^{\prime} \cdot z z^{\prime}=z,[w g, h]=1,
$$

and so $h w g$ is an involution. From $\left[h^{\prime}, h w g\right]=z z^{\prime}$ follows that

$$
\left\langle h^{\prime}, h w g\right\rangle \cong \mathrm{D}_{8} \text { with } \mathrm{Z}\left(\left\langle h^{\prime}, h w g\right\rangle\right)=\left\langle z z^{\prime}\right\rangle .
$$


But then $\mathrm{C}_{4} \cong\left\langle h^{\prime} h w g\right\rangle$ is not normal in $G$ since $\left[h^{\prime} h w g, h\right]=z$, a contradiction. We have proved that there is no such an element $w \in\langle a\rangle$. This implies

$$
n=2, o(a)=4, \exp (G)=4, a^{2}=z^{\prime},[a, g] \neq 1, \mathrm{Z}(G)=U=G^{\prime}=\Phi(G)
$$

and so $G$ is special of order $2^{6}$. It remains to determine $[a, g] \neq 1$.

Suppose that $[a, g]=z$. Then we get $(a g)^{2}=z^{\prime} \cdot z z^{\prime} \cdot z=1$ and so $a g$ is an involution. Since $\left[h^{\prime}, a g\right]=z^{\prime}$, we have $\left\langle h^{\prime}, a g\right\rangle \cong \mathrm{D}_{8}$ with $\mathrm{Z}\left(\left\langle h^{\prime}, a g\right\rangle\right)=\left\langle z^{\prime}\right\rangle$. But then $\mathrm{C}_{4} \cong\left\langle h^{\prime} a g\right\rangle$ is not normal in $G$ since $\left[h^{\prime} a g, h\right]=z$, a contradiction.

Suppose that $[a, g]=z z^{\prime}$. Then we get $\left(\text { gah }^{\prime}\right)^{2}=z z^{\prime} \cdot z^{\prime} \cdot z z^{\prime} \cdot z^{\prime}=1$ and so $g a h^{\prime}$ is an involution. Since $\left[g a h^{\prime}, h^{\prime}\right]=z^{\prime}$, we have $\left\langle g a h^{\prime}, h^{\prime}\right\rangle \cong$ $\mathrm{D}_{8}$ with $\mathrm{Z}\left(\left\langle g a h^{\prime}, h^{\prime}\right\rangle\right)=\left\langle z^{\prime}\right\rangle$. But then $\mathrm{C}_{4} \cong\left\langle g a h^{\prime} h^{\prime}\right\rangle=\langle g a\rangle$ is not normal in $G$ since $[g a, g]=z z^{\prime}$, a contradiction.

Hence we must have $[a, g]=z^{\prime}$ and so the structure of $G$ is uniquely determined. We set $h^{\prime}=i$ and so we get a special group $G$ of order $2^{6}$ given with:

$$
\begin{gathered}
G=(H \times\langle a\rangle)\langle g\rangle, \text { where } H=\left\langle h, i \mid h^{4}=i^{2}=1, h^{i}=h^{-1}, h^{2}=z\right\rangle \cong \mathrm{D}_{8}, \\
\langle a\rangle \cong \mathrm{C}_{4}, a^{2}=z^{\prime}, g^{2}=z z^{\prime},[g, h]=1,[g, i]=[g, a]=z^{\prime} .
\end{gathered}
$$

We have $G^{\prime}=\left\langle z, z^{\prime}\right\rangle \cong \mathrm{E}_{4},\langle h, i\rangle \cong \mathrm{D}_{8}$ is not normal in $G$ but $\langle h\rangle \unlhd G$, and $\langle i, a\rangle \cong \mathrm{C}_{2} \times \mathrm{C}_{4}$ is not normal in $G$ but $\langle a\rangle \unlhd G$. We have obtained the group stated in part (d) of our proposition.

It remains to be proved that this group $G$ satisfies our assumption (*). We first show that there are no involutions in $G-K$, where $K=H \times\langle a\rangle$. Indeed, suppose that $g h^{\alpha} i^{\beta} a^{\gamma}$ with $\alpha, \beta, \gamma \in\{0,1\}$ is an involution. Then we get

$$
1=\left(g h^{\alpha} i^{\beta} a^{\gamma}\right)^{2}=z z^{\prime} \cdot z^{\alpha} \cdot\left(z^{\prime}\right)^{\gamma} \cdot\left(z^{\prime}\right)^{\beta} \cdot\left(z^{\prime}\right)^{\gamma} \cdot z^{\alpha \beta}=z^{1+\alpha+\alpha \beta}\left(z^{\prime}\right)^{1+\beta},
$$

which implies $\beta=1$ and then we get $z=1$, a contradiction. We have proved that $\Omega_{1}(G)=L=H U$, where $U=\left\langle z, z^{\prime}\right\rangle$. There are exactly two conjugate classes of noncentral involutions in $G$ with representatives $i$ (4 conjugates) and $h i$ (4 conjugates) and we have

$$
\mathrm{C}_{G}(i)=\langle i, z\rangle \times\langle a\rangle \cong \mathrm{E}_{4} \times \mathrm{C}_{4} \text { and } \mathrm{C}_{G}(h i)=\langle h i, z\rangle \times\langle a\rangle \cong \mathrm{E}_{4} \times \mathrm{C}_{4} .
$$

Let $X$ be a noncyclic non-normal subgroup of order $\geq 2^{3}$ which contains more than one involution (so that $X \cong \mathrm{Q}_{8}$ is excluded). Then we have $G^{\prime}=U=\left\langle z, z^{\prime}\right\rangle \not \leq X$ and $|X|=2^{3}$ or $2^{4}$ (noting that all subgroups of order $\geq 2^{5}$ are normal in $G$ ).

First assume that $|X|=2^{4}$. In this case $X \not \leq K$ since $\Phi(K)=\left\langle z, z^{\prime}\right\rangle$ and $|K|=2^{5}$. We have $|X:(X \cap K)|=2$ and $|X \cap K|=2^{3}$. All elements in $X-K$ are of order 4 and so $\mho_{1}(X) \neq\{1\}$ and this implies that there is exactly one central involution $z_{0}$ in $G$ which is contained in $X \cap K$ and therefore we have $\mho_{1}(X)=\left\langle z_{0}\right\rangle$ and $\mathrm{d}(X)=3$. But $X \cap K$ must contain another involution $i^{\prime} \neq z_{0}$ which is noncentral in $G$ and we know (by the 
above) that $\mathrm{C}_{G}\left(i^{\prime}\right)=\mathrm{C}_{K}\left(i^{\prime}\right)$ is abelian. In particular, $X$ is nonabelian and $X^{\prime}=\left\langle z_{0}\right\rangle$. Because $\mathrm{d}(X)=3, X$ is not minimal nonabelian. Let $X_{0}$ be any minimal nonabelian subgroup in $X$. If $X_{0} \cong \mathrm{D}_{8}$, then (since there are no involutions in $X-K)$ we have $X_{0}=X \cap K$. Since $G^{\prime} \cong \mathrm{E}_{4}$, it follows that $X$ induces on $X_{0}$ only inner automorphisms of $X_{0}$ which implies that $\mathrm{C}_{X}\left(i^{\prime}\right) \not \leq K$, a contradiction. Hence each minimal nonabelian subgroup of $X$ is isomorphic to $Q_{8}$. By Corollary A.17.3 in [2], we get $X=\langle t\rangle \times Q$, where $t$ is an involution and $Q \cong \mathrm{Q}_{8}$ with $\mathrm{Z}(Q)=X^{\prime}=\left\langle z_{0}\right\rangle$. Thus $t$ is a noncentral involution in $G$, contrary to the fact that $\mathrm{C}_{G}(t)$ must be abelian.

We have proved that $|X|=2^{3}$ and assume first that $X \not K K$. Since $X$ contains more than one involution, it follows that $X \cap K$ contains a noncentral involution $i^{\prime}$ of $G$. We know that $\mathrm{C}_{G}\left(i^{\prime}\right) \leq K$ and so $X$ is nonabelian. But then $X \cong \mathrm{D}_{8}$ which is not possible since there are no involutions in $X-K$. We have proved that $X \leq K$.

If $X \cong \mathrm{E}_{8}$, then $X \leq L$, where $L=H \times\left\langle z^{\prime}\right\rangle$. But then $X \geq\left\langle z, z^{\prime}\right\rangle=G^{\prime}$, a contradiction. It follows that either $X \cong \mathrm{D}_{8}$ or $X \cong \mathrm{C}_{4} \times \mathrm{C}_{2}$. First assume that $X \cong \mathrm{D}_{8}$. Because in this case $\Omega_{1}(X)=X$ and $\Omega_{1}(K)=L$, it follows that $X \leq L$. But then $X$ is conjugate in $G$ to $H=\langle h, i\rangle$ or to $H^{*}=\left\langle h z^{\prime}, i\right\rangle$, where both $\langle h\rangle$ and $\left\langle h z^{\prime}\right\rangle$ are normal in $G$.

Finally, suppose that $X \cong \mathrm{C}_{4} \times \mathrm{C}_{2}$. Because in this case $\{1\} \neq \mho_{1}(X) \leq$ $\left\langle z, z^{\prime}\right\rangle$, it follows that $X$ contains exactly one central involution of $G$ and two noncentral involutions of $G$. Then $X$ is conjugate in $G$ to $X_{1}=\langle i\rangle \times\langle v\rangle$ or to $X_{2}=\langle h i\rangle \times\langle w\rangle$, where $\langle v\rangle \cong\langle w\rangle \cong \mathrm{C}_{4}$. Since

$$
X_{1} \leq \mathrm{C}_{G}(i)=\mathrm{C}_{K}(i)=\langle i, z\rangle \times\langle a\rangle,
$$

we get $X_{1}=\langle i\rangle \times\langle a\rangle$ or $X_{1}=\langle i\rangle \times\langle a z\rangle$. Similarly,

$$
X_{2} \leq \mathrm{C}_{G}(h i)=\mathrm{C}_{K}(h i)=\langle h i, z\rangle \times\langle a\rangle,
$$

gives $X_{2}=\langle h i\rangle \times\langle a\rangle$ or $X_{2}=\langle h i\rangle \times\langle a z\rangle$. On the other hand, we see that $\langle a\rangle \unlhd G$ and $\langle a z\rangle \unlhd G$ and we are done. Our proposition is completely proved.

Proposition 4.6. Suppose that we have the case (b1) of Proposition 3. Then $H$ possesses exactly one $G$-invariant cyclic subgroup of index $p$.

Proof. We have $H \cong \mathrm{M}_{p^{n}}, n \geq 3$, ( if $p=2$, then $n \geq 4$ ) or $H$ is abelian of type $\left(p^{s}, p\right), s \geq 2$. Set $H_{0}=\Omega_{1}(H)$ and then we have

$$
H_{0} \cong \mathrm{E}_{p^{2}}, \mathrm{~N}_{G}\left(H_{0}\right)=\mathrm{N}_{G}(H)=K,|G / K|=p, U_{0}=U \cap H=\langle z\rangle \leq \mathrm{Z}(G),
$$

and let $g \in G-K$. Note that $H$ has exactly $p$ cyclic subgroups of index $p$. By Proposition 4, we have $G^{\prime} \leq U$ and so we get $[K, H] \leq H \cap U=U_{0}=\langle z\rangle$. This implies that each cyclic subgroup of index $p$ in $H$ is normal in $K$. Assume, by way of contradiction, that $H$ does not have any $G$-invariant cyclic subgroup of index $p$. Since $H \cap H^{g}$ is a cyclic subgroup of index $p$ in $H$, there is a 
cyclic subgroup $\langle h\rangle$ of index $p$ in $H$ such that $\langle h\rangle^{g}=\langle h t\rangle$ for some element $t \in H_{0}-\langle z\rangle$. Then we get

$$
h^{g}=h t v \text { with some } v \in\left\langle(h t)^{p}\right\rangle=\left\langle h^{p}\right\rangle .
$$

In that case we get

$$
h^{-1} h^{g}=[h, g]=t v \in U \cap H=\langle z\rangle .
$$

Since $v \in\left\langle h^{p}\right\rangle$ and (by Proposition 3(b1)) $\left\langle h^{p}\right\rangle \geq\langle z\rangle$, it follows that $t \in\left\langle h^{p}\right\rangle$, a contradiction. Since $H$ is not normal in $G$, then clearly $H$ possesses exactly one $G$-invariant cyclic subgroup of index $p$ and we are done.

Proposition 4.7. Suppose that we have the case (b1) of Proposition 3 and assume in addition that $K / H_{0}$ is Hamiltonian (and so $p=2$ ), where $H_{0}=\Omega_{1}(H) \cong \mathrm{E}_{4}$, and that $G$ does not possess any non-normal subgroup isomorphic to $\mathrm{D}_{8}$. Then $G$ is of order $2^{7}$ and class 2 which has a normal subgroup $K$ of index 2 , where

$$
K=(\langle h\rangle \times Q)\langle t\rangle \text { with }\langle h\rangle \cong \mathrm{C}_{4}, h^{2}=z, Q=\langle a, b\rangle \cong \mathrm{Q}_{8}, Q^{\prime}=\langle u\rangle,
$$

$t$ is an involution commuting with $h$ and $a$ and $[b, t]=z$. There is an element $g \in G-K$ such that either

(a) $g^{2}=u z, g$ centralizes $Q,[g, h]=z,[g, t]=u$

(and here $G$ is a special group with $G^{\prime}=\langle u, z\rangle \cong \mathrm{E}_{4}$ and $\Omega_{1}(G)=$ $\left.G^{\prime} \times\langle t\rangle \cong \mathrm{E}_{8}\right)$

or

(b) $g^{2}=h, g$ centralizes $Q,[g, t]=u z$

(and here $G$ is of exponent 8 with $G^{\prime}=\langle u, z\rangle \cong \mathrm{E}_{4}, \mathrm{Z}(G)=$ $G^{\prime}\langle h\rangle \cong \mathrm{C}_{4} \times \mathrm{C}_{2}, \Omega_{1}(G)=G^{\prime} \times\langle t\rangle \cong \mathrm{E}_{8}$ and $\left.\Omega_{2}(G)=K\right)$.

Conversely, the above two groups satisfy our assumption $(*)$.

Proof. We have

$$
\begin{gathered}
H_{0}=\Omega_{1}(H) \cong \mathrm{E}_{4}, \mathrm{~N}_{G}\left(H_{0}\right)=\mathrm{N}_{G}(H)=K,|G: K|=2, \\
\mathrm{E}_{8} \cong S=H_{0} U \unlhd G, U \cap H_{0}=U \cap H=U_{0}=\langle z\rangle \leq \mathrm{Z}(G),
\end{gathered}
$$

and $K / H_{0}$ is Hamiltonian. By Proposition 4, we have $G^{\prime} \leq U$ and this gives

$$
\left(K / H_{0}\right)^{\prime}=S / H_{0}=\mho_{1}\left(K / H_{0}\right),
$$

and so $\exp (K)=4$ and $H \cong \mathrm{C}_{4} \times \mathrm{C}_{2}$. By Proposition 3(b1), $L=H U$ is abelian of type $(4,2,2), \mho_{1}(L)=\mho_{1}(H)=U_{0}=\langle z\rangle$ and so we have $S=\Omega_{1}(L)=\Omega_{1}(K)$.

Let $Q / H_{0}$ be an ordinary quaternion subgroup of $K / H_{0}$. Since

$$
\left(Q / H_{0}\right)^{\prime}=\left(K / H_{0}\right)^{\prime}=S / H_{0},
$$

it follows that $S<Q$. Also, $S / H_{0}$ is a unique subgroup of order 2 in $Q / H_{0}$ and so we have $Q \cap H=H_{0}$ and $Q \cap L=S$. Since $Q / H_{0} \cong \mathrm{Q}_{8}$ is isomorphic 
to a subgroup of $K / H$, Proposition 2 implies that $K / H \cong \mathrm{Q}_{8}$ and so we get $K=H Q$ with $H \cap Q=H_{0}$.

We have $\left|Q: \mathrm{C}_{Q}\left(H_{0}\right)\right| \leq 2$ and so if $a \in \mathrm{C}_{Q}\left(H_{0}\right)-S$, then $a^{2} \in S-H_{0}$ and so $A=\langle a\rangle \times H_{0} \cong \mathrm{C}_{4} \times \mathrm{E}_{4}$ (containing $U$ ) is an abelian maximal subgroup of $Q, A \unlhd G$ and we get $\mho_{1}(A)=\left\langle a^{2}\right\rangle \leq \mathrm{Z}(G)$ and $\mathrm{E}_{4} \cong\left\langle a^{2}, z\right\rangle \unlhd G$. On the other hand, $G / K$ acts on the three maximal subgroups of $S$ which contain $\langle z\rangle \leq \mathrm{Z}(G)$ fixing $U$ and fusing the other two (since $\mathrm{N}_{G}\left(H_{0}\right)=K$ ) and so we get $\left\langle a^{2}, z\right\rangle=U$ and $U \leq \mathrm{Z}(G)$. In particular, $G$ is of class 2 with an elementary abelian commutator subgroup of order $\leq 4$ (contained in $U$ ) and this implies that $\mho_{1}(G) \leq \mathrm{Z}(G)$. Indeed, if $x, y \in \bar{G}$, then we have $\left[x^{2}, y\right]=[x, y]^{2}=1$. We have $\mho_{1}(K) \leq S$ and since $S \cap \mathrm{Z}(G)=U$, we get $\mho_{1}(K) \leq U$ and so $\Phi(K)=U$. For each element $k \in K-L$, we have $k^{2} \in U-\langle z\rangle$.

By Proposition $6, H$ possesses exactly one cyclic subgroup $\langle h\rangle$ of index 2 which is normal in $G$ and we have $h^{2}=z$. Note that for an element $u \in U-\langle z\rangle$, the cyclic subgroup $\langle h u\rangle \cong \mathrm{C}_{4}$ is also normal in $G$. But the abelian normal subgroup $L$ possesses exactly four cyclic subgroups of order 4 and so the other two cyclic subgroups of order 4 in $L$ (which are distinct from $\langle h\rangle$ and $\langle h u\rangle)$ must be fused in $G$. Indeed, if $t \in H_{0}-\langle z\rangle$ and $g \in G-K$, then we have $t^{g}=t u$ for some $u \in U-\langle z\rangle$ and so we get $\langle h t\rangle^{g}=\langle h t u\rangle$.

By Proposition $4, G / U$ is abelian and so $G / L$ is abelian and $K / L \cong \mathrm{E}_{4}$. Assume that $G / L$ is not elementary abelian. Then there is an element $x \in$ $G-K$ such that $x^{2} \in K-L$. But then $x^{2} \in \mathrm{Z}(G)$, contrary to the fact that $K / H \cong \mathrm{Q}_{8}$. Hence we have $G / L \cong \mathrm{E}_{8}$. For any $g \in G-K$, we have $g^{2} \in L \cap \mathrm{Z}(G)$ and so either $g^{2} \in U$ or $g^{2} \in L-S$ and in the second case we have either $g^{2} \in\langle h\rangle$ or $g^{2} \in\langle h u\rangle$ with $u \in U-\langle z\rangle$. Note that $H_{1}=\langle h u, t\rangle$ is also a maximal non-normal subgroup in $G$ with $\Omega_{1}\left(H_{1}\right)=\Omega_{1}(H)=\langle z, t\rangle$. Indeed, if $H_{1}$ is not maximal non-normal, then let $H_{1}^{*}$ containing $H_{1}$ be a maximal non-normal subgroup in $G$. Since $\exp (G) \leq 8$ and $\exp (K)=4$, it follows that $H_{1}^{*} \cong \mathrm{C}_{8} \times \mathrm{C}_{2}$ or $H_{1}^{*} \cong \mathrm{M}_{16}$ and so $H_{1}^{*} \not K K$. But we have

$$
\Omega_{1}\left(H_{1}^{*}\right)=\Omega_{1}\left(H_{1}\right)=H_{0}=\langle z, t\rangle
$$

and so we get $H_{0} \unlhd G$, a contradiction. Thus, in case that we have an element $g \in G-K$ with $g^{2} \in\langle h u\rangle$, we replace $H$ with $H_{1}$ (and write again $H$ instead of $H_{1}$ ) so that we may assume from the start that $g^{2} \in\langle h\rangle$ and then (by a suitable choice of a generator of $\langle g\rangle$ ) we have $g^{2}=h$.

Let $k$ be any element in $K-L$ which commutes with $t \in H_{0}-\langle z\rangle$. Then we have $k^{2} \in U-\langle z\rangle$ so that $\langle k, t\rangle \cong \mathrm{C}_{4} \times \mathrm{C}_{2}$. We claim that in that case at least one of cyclic subgroups $\langle k\rangle$ or $\langle k t\rangle$ is normal in $G$. If $\langle k, t\rangle \unlhd G$, then both $\langle k\rangle$ and $\langle k t\rangle$ are normal in $G$ because $G^{\prime} \leq U$. (If there is $x \in G$ such that $k^{x}=k t$ or $k^{x}=k^{-1} t$, then we have either $t \in G^{\prime}$ or $k^{2} t \in G^{\prime}$ and so $t \in U$, a contradiction.) If $\langle k, t\rangle$ is not normal in $G$, then it is easy to see that $\langle k, t\rangle$ is a maximal non-normal subgroup in $G$. Indeed, if $\left.H^{*}\right\rangle\langle k, t\rangle$ is a maximal non-normal subgroup in $G$, then by Proposition $3, H^{*} \cong \mathrm{C}_{8} \times \mathrm{C}_{2}$ 
or $H^{*} \cong \mathrm{M}_{16}$ (noting that $\exp (G) \leq 8$ ) and so $k$ or $k t$ is a square in $H^{*}$ and therefore $k$ or $k t$ is contained in $\mathrm{Z}(G)$, contrary to the fact that $K / H \cong \mathrm{Q}_{8}$. Hence $\langle k, t\rangle$ is a maximal non-normal subgroup in $G$ and so, by Proposition 6 , one of $\langle k\rangle$ or $\langle k t\rangle$ is normal in $G$. Since $\langle k, t\rangle \cap\langle h\rangle=\{1\}$ and $\langle h\rangle \unlhd G$, we see that $k$ or $k t$ commutes with $h$. But $t$ commutes with $h$ and so in any case $k$ commutes with $h$. We have proved that whenever an element $k \in K-L$ commutes with $t \in H_{0}-\langle z\rangle$, then $k$ also commutes with $h$.

Suppose, by way of contradiction, that $t \in \mathrm{Z}(Q)$. Let $a, b \in Q-S$ be such that $\langle a, b\rangle$ covers $Q / S$ and set $a^{2}=u \in U-\langle z\rangle$. By the above, both $a$ and $b$ commute with $h$. We have $[a, b] \in U-\langle z\rangle$ and so $[a, b] \in\{u, u z\}$. Suppose at the moment that $[a, b]=u z$. By the previous paragraph, we know that $\langle a\rangle$ or $\langle a t\rangle$ is normal in $G$. On the other hand, we have

$$
a^{b}=a(u z),(a t)^{b}=(a t)(u z) \text { with } a^{2}=(a t)^{2}=u,
$$

and so both $\langle a\rangle$ and $\langle a t\rangle$ are non-normal in $G$, a contradiction. Thus, we must have $[a, b]=u$. Considering the subgroup $\langle a h\rangle \times\langle t\rangle$, we know that one of $\langle a h\rangle$ or $\langle a h t\rangle$ must be normal in $G$. But we have

$$
(a h)^{2}=(a h t)^{2}=u z,(a h)^{b}=(a h) u,(a h t)^{b}=(a h t) u,
$$

and so both $\langle a h\rangle$ and $\langle a h t\rangle$ are non-normal in $G$, a contradiction.

We have proved that $t \notin \mathrm{Z}(Q)$. Then we have $\left|Q: \mathrm{C}_{Q}(t)\right|=2$. Let $a \in \mathrm{C}_{Q}(t)-S$ and $b \in Q-\mathrm{C}_{Q}(t)$ so that

$$
\langle a, b\rangle \text { covers } Q / S,[a, b] \in U-\langle z\rangle,[a, h]=1, \text { and }[b, t]=z \text {. }
$$

In particular, we get $Q^{\prime}=G^{\prime}=U$ and we set $a^{2}=u \in U-\langle z\rangle$. If $[a, b]=u z$, then we replace $a$ with $a^{\prime}=a t$ (noting that $\left[a^{\prime}, h\right]=1$ and $\left(a^{\prime}\right)^{2}=u$ ) and then we get $\left[a^{\prime}, b\right]=[a t, b]=u z \cdot z=u$. We write $a$ instead $a^{\prime}$ so that we may assume from the start that $[a, b]=u$. If $b^{2}=u z$, then we replace $b$ with $b^{\prime}=b t$ (noting that $\left[a, b^{\prime}\right]=[a, b t]=u$ and $\left[b^{\prime}, t\right]=[b t, t]=z$ ) and we obtain

$$
\left(b^{\prime}\right)^{2}=(b t)^{2}=b^{2} t^{2}[t, b]=u z \cdot z=u \text {. }
$$

Hence writing $b$ instead of $b^{\prime}$, we may assume from the start that $b^{2}=u$. We have obtained that $Q^{*}=\langle a, b\rangle \cong \mathrm{Q}_{8}$. Since $(a t)^{b}=(a t)(u z)$ and $(a t)^{2}=u$, we see that $\langle a t\rangle$ is not normal in $G$. This implies that $\langle a\rangle$ is normal in $G$. Also note that $b$ has four conjugates in $Q$ and $Q \unlhd G$. Since $\left|G^{\prime}\right|=4, b$ has exactly four conjugates in $G$ and so $\mathrm{C}_{G}(b)$ must cover $G / Q$. Let $g \in \mathrm{C}_{G}(b)-K$ and we know that $g$ normalizes $\langle a\rangle$. If $a^{g}=a^{-1}=a u$, then we replace $g$ with $g^{\prime}=g b \in G-K$ so that

$$
a^{g^{\prime}}=a^{g b}=(a u)^{b}=(a u) u=a .
$$

Noting that $g^{\prime}$ also commutes with $b$, we may write $g$ instead of $g^{\prime}$ so that we may assume from the start that $g \in G-K$ centralizes $Q^{*}=\langle a, b\rangle$. Since $t^{b}=t z$ and $t^{g}=t u^{\prime}$ with some $u^{\prime} \in U-\langle z\rangle$, it follows that the conjugate class of $t$ in $G$ contains four elements (and they all lie in $S-U$ ). 
Now it is easy to see that there are no involutions contained in $G-K$ and so we have $\Omega_{1}(G)=S=G^{\prime} \times\langle t\rangle \cong \mathrm{E}_{8}$. Indeed, assume that there is an involution $i \in G-K$. Then we have $D=\langle i, t\rangle \cong \mathrm{D}_{8}$ and by our assumption we have $D \unlhd G$. Since $G^{\prime} \cong \mathrm{E}_{4}$ is elementary abelian, each element in $G$ induces on $D$ an inner automorphism of $D$. In particular, both four-subgroups in $D$ are normal in $G$. But then $t$ would have only two conjugates in $G$, a contradiction.

It remains to determine:

$$
g^{2}, h^{g}=h z^{\epsilon}, h^{b}=h z^{\eta}, \text { and } t^{g}=t u z^{\zeta}, \text { where } \epsilon, \eta, \zeta \in\{0,1\} .
$$

Considering the subgroup $\langle a h\rangle \times\langle t\rangle$, we know (by the above) that at least one of the cyclic subgroups $\langle a h\rangle$ or $\langle a h t\rangle$ must be normal in $G$. Since

$$
\langle h, a, t\rangle=\langle h\rangle \times\langle a\rangle \times\langle t\rangle \cong \mathrm{C}_{4} \times \mathrm{C}_{4} \times \mathrm{C}_{2}
$$

is abelian, it is enough to consider the action of elements $b$ and $g$ on these cyclic subgroups. We have

$$
\begin{gathered}
(a h)^{2}=(a h t)^{2}=u z, \text { and }(a h)^{b}=(a h) u z^{\eta},(a h)^{g}=(a h) z^{\epsilon}, \\
(a h t)^{b}=(a h t) u z^{\eta+1},(a h t)^{g}=(a h t) u z^{\epsilon+\zeta} .
\end{gathered}
$$

If $\eta=1$, then $(a h t)^{b}=(a h t) u$ and so $\langle a h t\rangle$ is not normal in $G$. Then we must have $\langle a h\rangle \unlhd G$ and so we get $\epsilon=0$.

If $\eta=0$, then $(a h)^{b}=(a h) u$ and so $\langle a h\rangle$ is not normal in $G$. Then we must have $\langle a h t\rangle \unlhd G$ which gives $\epsilon+\zeta=1$.

(i) First assume that $g^{2} \in\{u, z, u z\}$. If $\epsilon=0$, then $h^{g}=h$ and so $g$ centralizes $\langle h\rangle \times\langle a\rangle \cong \mathrm{C}_{4} \times \mathrm{C}_{4}$ and then there is an involution in $g\langle h, a\rangle$, a contradiction. Hence we must have $\epsilon=1$. By the above, we get $\eta=0$ and $\zeta=0$. Hence we have in this case

$$
h^{g}=h z, h^{b}=h \text {, and } t^{g}=t u .
$$

If $g^{2}=u$, then $[g, a]=1$ implies that $g a$ is an involution, a contradiction. If $g^{2}=z$, then $(t b)^{2}=u z$ and

$$
(g t b)^{2}=z \cdot u z \cdot[t b, g]=u \cdot u=1
$$

so that $g t b$ is an involution, a contradiction. Hence we must have $g^{2}=u z$. The structure of $G$ is determined as given in part (a) of our proposition. We check that there are no involutions in $G-K$. Indeed, assume that $g h^{\alpha} t^{\beta} a^{\gamma} b^{\delta} u^{\prime}$ with $u^{\prime} \in U=\mathrm{Z}(G)$ and $\alpha, \beta, \gamma, \delta \in\{0,1\}$, is an involution. Then we get

$$
1=\left(g h^{\alpha} t^{\beta} a^{\gamma} b^{\delta} u^{\prime}\right)^{2}=u^{1+\beta+\gamma+\delta+\gamma \delta} z^{1+\beta \delta},
$$

and so $\beta=\delta=1$, which gives $u=1$, a contradiction.

It remains to prove that this special group $G$ of order $2^{7}$ satisfies our condition $(*)$. Let $X$ be a noncyclic and non-normal subgroup of order $\geq 2^{3}$ which has more than one involution. Then $|X \cap S|=4$ and $X \cap U=\left\langle u^{\prime}\right\rangle$, where $u^{\prime}$ is a central involution and $S=\Omega_{1}(G)=U \times\langle t\rangle$. But all four 
involutions in $S-U$ are conjugate in $G$ noting that $\mathrm{C}_{G}(t)=\langle h\rangle \times\langle a\rangle \times\langle t\rangle$. Therefore we may assume that $t \in X$ and so we have $\Omega_{1}(X)=\left\langle t, u^{\prime}\right\rangle=X \cap S$. We have $X \leq \mathrm{N}_{G}\left(\left\langle t, u^{\prime}\right\rangle\right)$ and since $\Omega_{1}(X)$ contains at most two conjugates $t$ and $t u^{\prime}$ of $t$, it follows that $X$ cannot cover $G / \mathrm{C}_{G}(t)$. Therefore we have either $X \leq \mathrm{C}_{G}(t)$ or $X \not \leq \mathrm{C}_{G}(t)$ in which case we must have one of the three possibilities: $X \leq \mathrm{C}_{G}(t)\langle b\rangle$ or $X \leq \mathrm{C}_{G}(t)\langle g\rangle$ or $X \leq \mathrm{C}_{G}(t)\langle b g\rangle$.

First assume that $X \not \leq \mathrm{C}_{G}(t)$ and then we have three subcases.

(1) If $X \leq \mathrm{C}_{G}(t)\langle b\rangle$, then $t^{b}=t z$ and so $u^{\prime}=z$. If $x \in X-\mathrm{C}_{G}(t)$, then $x^{2} \in U-\langle z\rangle$, which gives $X \geq U=G^{\prime}$, a contradiction.

(2) Assume that $X \leq \mathrm{C}_{G}(t)\langle g\rangle$ and then we have $t^{g}=t u$ and so $u^{\prime}=u$. If in this case $x \in X-\mathrm{C}_{G}(t)$, then we have

$$
x=g a^{\alpha} t^{\beta} h^{\gamma} u^{\prime \prime}\left(u^{\prime \prime} \in U, \alpha, \beta, \gamma \in\{0,1\}\right) \text { and then } x^{2}=u^{1+\alpha+\beta} z,
$$

which gives that $X \geq U=G^{\prime}$, a contradiction.

(3) Suppose that $X \leq \mathrm{C}_{G}(t)\langle b g\rangle$ and then we have $t^{b g}=t u z$ and so $u^{\prime}=u z$. If in this case $x \in X-\mathrm{C}_{G}(t)$, then we have

$$
x=b g a^{\alpha} t^{\beta} h^{\gamma} u^{\prime \prime}\left(u^{\prime \prime} \in U, \alpha, \beta, \gamma \in\{0,1\}\right) \text { and then } x^{2}=u^{\beta} z^{1+\beta} .
$$

If $\beta=0$, then $x^{2}=z$. If $\beta=1$, then $x^{2}=u$. In any case we get $X \geq U=G^{\prime}$, a contradiction.

Now assume $X \leq \mathrm{C}_{G}(t)=(\langle h\rangle \times\langle a\rangle) \times\langle t\rangle$. Since $X \nsupseteq G^{\prime}=U$, we have

$X \in\left\{\left\langle h u^{\mu}\right\rangle \times\langle t\rangle,\left\langle a z^{\nu}\right\rangle \times\langle t\rangle,\left\langle a h z^{\sigma}\right\rangle \times\langle t\rangle\right.$, where $\left.\mu, \nu, \sigma \in\{0,1\}.\right\}$

If $X=\left\langle h u^{\mu}\right\rangle \times\langle t\rangle$, then we have $\left\langle h u^{\mu}\right\rangle \unlhd G$.

If $X=\left\langle a z^{\nu}\right\rangle \times\langle t\rangle$, then $\left\langle a z^{\nu}\right\rangle \unlhd G$.

If $X=\left\langle a h z^{\sigma}\right\rangle \times\langle t\rangle$, then $\left\langle a h z^{\sigma} t\right\rangle \unlhd G$ since

$$
\left(a h z^{\sigma} t\right)^{2}=u z,\left[a h z^{\sigma} t, b\right]=u z, \text { and }\left[a h z^{\sigma} t, g\right]=u z .
$$

We have proved that the condition $(*)$ is satisfied because for example $\langle h\rangle \times\langle t\rangle$ is not normal in $G$ (noting that $t^{g}=t u$ ).

(ii) Assume that $g^{2}=h$. In this case we have $h \in \mathrm{Z}(G)$ and this gives $\epsilon=0$ and $\eta=0$. It follows (from the above) that $\zeta=1$ and so we have $t^{g}=t u z$. The structure of $G$ is determined as given in part (b) of our proposition. For each $k \in K$ we have $(g k)^{4}=g^{4}=z$. Thus, all elements in $G-K$ are of order 8 and so we have $\Omega_{1}(G)=S=G^{\prime} \times\langle t\rangle \cong \mathrm{E}_{8}$.

Conversely, let $X$ be a noncyclic and non-normal subgroup of order $\geq 2^{3}$ in $G$ which has more than one involution. Since four noncentral involutions in $S-U$ form a single conjugate class in $G$, it follows that we may assume $t \in X$. In addition, $X$ contains exactly one central involution $u^{\prime} \in U$ so that we have $\Omega_{1}(X)=\left\langle t, u^{\prime}\right\rangle$.

First suppose that $X \not K K$ so that $X$ contains elements of order 8 which implies that $z \in X$ and so we have $\Omega_{1}(X)=\langle t, z\rangle=H_{0}$. But then $H_{0} \unlhd G$, contrary to $t^{g}=t u z$. 
We have proved that we must have $X \leq K$. Suppose that

$$
X \not \leq \mathrm{C}_{G}(t)=(\langle h\rangle \times\langle a\rangle) \times\langle t\rangle \text { and let } x \in X-\mathrm{C}_{G}(t) .
$$

Then we have $t^{x}=t z$ and $x^{2} \in U-\langle z\rangle$ and so $X \geq U=\langle u, z\rangle=G^{\prime}$, a contradiction.

Thus, we must have $X \leq \mathrm{C}_{G}(t)$ and since $\langle u, z\rangle \not X X$, we get $X \cong \mathrm{C}_{4} \times \mathrm{C}_{2}$. We have three subcases.

If $X=\left\langle h u^{\mu}\right\rangle \times\langle t\rangle(\mu \in\{0,1\})$, then we have $\left\langle h u^{\mu}\right\rangle \unlhd G$.

If $X=\left\langle a z^{\nu}\right\rangle \times\langle t\rangle(\nu \in\{0,1\})$, then $\left\langle a z^{\nu}\right\rangle \unlhd G$.

If $X=\left\langle a h z^{\sigma}\right\rangle \times\langle t\rangle(\sigma \in\{0,1\})$, then $\left\langle a h z^{\sigma} t\right\rangle \unlhd G$ since

$$
\left(a h z^{\sigma} t\right)^{2}=u z,\left[a h z^{\sigma} t, b\right]=u z \text {, and }\left[a h z^{\sigma} t, g\right]=u z \text {. }
$$

We have proved that the condition $(*)$ is satisfied because for example $\langle h\rangle \times\langle t\rangle$ is not normal in $G$ (noting that $t^{g}=t u z$ ). Our proposition is completely proved.

Proposition 4.8. Suppose that our group $G$ has the commutator group $G^{\prime}$ of order $p$. Then we have $|G: \mathrm{Z}(G)|=p^{2}, \mathrm{Z}(G)$ is of rank $2, \Omega_{1}(G) \not \mathbf{Z}(G)$ and $\mathrm{Z}(G)$ possesses cyclic subgroups of order $\geq p^{2}$ which do not contain $G^{\prime}$.

Conversely, all these groups satisfy our condition $(*)$.

Proof. By Propositions 2 and 3, we must be in case (b1) of Proposition 3 , where $H$ is abelian of type $\left(p^{s}, p\right), s \geq 2, L=H U$ is abelian of type $\left(p^{s}, p, p\right)$ with $\mho_{1}(L)=\mho_{1}(H) \geq U_{0}=H \cap U=\langle z\rangle \leq \mathrm{Z}(G)$. By Proposition $3, G^{\prime}$ covers $U /\langle z\rangle$ and so we may set $G^{\prime}=\langle u\rangle$, where $u \in U-\langle z\rangle$ so that $U \leq \mathrm{Z}(G)$. We have $\mathrm{N}_{G}\left(H_{0}\right)=\mathrm{N}_{G}(H)=K$, where $H_{0}=\Omega_{1}(H) \cong \mathrm{E}_{p^{2}}$, $S=H_{0} U \cong \mathrm{E}_{p^{3}}$ and $S=\Omega_{1}(K)$. Note that $G / K \cong \mathrm{C}_{p}$ acts transitively on $p$ subgroups of order $p^{2}$ in $S$ which contain $\langle z\rangle$ and which are distinct from $U$ and so we have $\mathrm{Z}(G) \cap S=U$. Since $\mathrm{Z}(G) \leq K$, it follows that $\mathrm{Z}(G)$ is of rank 2 and $\Omega_{1}(G) \not Z \mathrm{Z}(G)$. By Proposition 3, $G$ does not possess any non-normal subgroup isomorphic to $\mathrm{D}_{8}$ and so by Proposition $7, K / H_{0}$ is abelian. This implies that $K$ is abelian and so Lemma 1.1 in [1] gives at once that $|G: \mathrm{Z}(G)|=p^{2}$. By Proposition $6, H$ has exactly one $G$-invariant cyclic subgroup $\langle h\rangle \cong \mathrm{C}_{p^{s}}, s \geq 2$, where $\langle h\rangle \cap U=\langle z\rangle$ and so $G^{\prime} \not\langle\langle h\rangle$. But we have

$$
[G,\langle h\rangle] \leq\langle h\rangle \cap G^{\prime}=\{1\} \text { and so }\langle h\rangle \leq \mathrm{Z}(G) .
$$

We have proved that $\mathrm{Z}(G)$ contains cyclic subgroups of order $\geq p^{2}$ which do not contain $G^{\prime}$. We have obtained the groups stated in our proposition.

Conversely, let $X$ be any noncyclic and non-normal subgroup of order $\geq p^{3}$ in a group $G$ described in our proposition. Since $G^{\prime} \not \leq X$, it follows that $X$ is abelian and so $X$ does not cover $G / \mathrm{Z}(G)$ and $X \not \leq \mathrm{Z}(G)$. We get $|X:(X \cap \mathrm{Z}(G))|=p$ and $X_{0}=X \cap \mathrm{Z}(G)$ is cyclic (since $\mathrm{E}_{p^{2}} \cong \Omega_{1}(\mathrm{Z}(G)$ ) contains $\left.G^{\prime}\right)$. For any $g \in G$ with $X^{g} \neq X$, we see that $X \cap X^{g}=X_{0}$ is cyclic. Let $\langle k\rangle$ be a maximal cyclic subgroup of order $\geq p^{2}$ in $\mathrm{Z}(G)$ which does not 
contain $G^{\prime}$ and let $i$ be an element of order $p$ in $\Omega_{1}(G)-\mathrm{Z}(G)$. Then $\langle k\rangle \times\langle i\rangle$ does not contain $G^{\prime}$ and so $\langle k\rangle \times\langle i\rangle$ is a maximal non-normal subgroup of $G$ of type $\left(p^{r}, p\right), r \geq 2$. Indeed, if $\langle k\rangle \times\langle i\rangle \unlhd G$, then

$$
[G,(\langle k\rangle \times\langle i\rangle)] \leq(\langle k\rangle \times\langle i\rangle) \cap G^{\prime}=\{1\}
$$

and so $i \in \mathrm{Z}(G)$, a contradiction. The maximality of the cyclic subgroup $\langle k\rangle$ in $\mathrm{Z}(G)$ also shows that $\langle k\rangle \times\langle i\rangle$ is a maximal non-normal subgroup in $G$ and we are done.

Proposition 4.9. Suppose that we have the case (b1) of Proposition 3, where $H \cong \mathrm{M}_{p^{n}}, n \geq 3$ (if $p=2$, then $n \geq 4$ ), $G$ is of class 3 and $G$ does not have non-normal subgroups isomorphic to $\mathrm{D}_{8}$ or such one which lead to the case (b2) of Proposition 3. Then we have $p=2, G$ has the following subgroup of index 2 :

$$
\mathrm{M}_{2^{n+1}} \cong\left\langle g, u \mid g^{2^{n}}=u^{2}=1,[g, u]=z=g^{2^{n-1}}\right\rangle, n \geq 4,
$$

and $G=\langle g, u\rangle\langle t\rangle$, where $t$ is an involution with $[g, t]=u$ and $[u, t]=1$.

We have

with

$$
|G|=2^{n+2}, n \geq 4,
$$

$$
\begin{gathered}
G^{\prime}=\langle u, z\rangle \cong \mathrm{E}_{4},\left[G, G^{\prime}\right]=\langle z\rangle, \Omega_{1}(G)=\langle u, z, t\rangle \cong \mathrm{E}_{8}, \\
\mathrm{Z}(G)=\left\langle g^{4}\right\rangle \cong \mathrm{C}_{2^{n-2}} \text { and }\left\langle g^{2}, t\right\rangle \cong \mathrm{M}_{2^{n}}
\end{gathered}
$$

is a non-normal subgroup in $G$ with $\left\langle g^{2}\right\rangle \unlhd G$.

Conversely, these groups satisfy the condition $(*)$.

Proof. By Proposition $4, G^{\prime} \leq U$ and so we have $G^{\prime}=U \not \leq \mathrm{Z}(G)$. Also, Proposition 7 implies that $K / \Omega_{1}(H)$ is abelian, where $\Omega_{1}(H) \cong \mathrm{E}_{p^{2}}$ and so we have $K^{\prime}=H^{\prime}=\langle z\rangle \leq \mathrm{Z}(G)$. By Proposition $2, K / H$ is cyclic of order $\geq p$. Finally, Proposition 3 also implies that $U=\Omega_{1}(\mathrm{Z}(L))$, where $L=H U \unlhd G$. By Proposition 6, $H$ possesses a $G$-invariant cyclic subgroup $\langle h\rangle$ of index $p$ and there is an element $t$ of order $p$ in $H-\langle h\rangle$ so that $\langle[h, t]\rangle=\langle z\rangle$. For any $g \in G-K$, we have $t^{g}=t u^{\prime}$ for some $u^{\prime} \in U-\langle z\rangle$, where $G / K \cong \mathrm{C}_{p}$, $S=\langle t\rangle U \cong \mathrm{E}_{p^{3}}$ is normal in $G$ and $S=\Omega_{1}(K)$. It follows that all $p^{2}$ subgroups of order $p$ contained in $(S-U) \cup\{1\}$ form a single conjugate class in $G$.

Since $K^{\prime}=H^{\prime}$, we get $\mho_{1}(K) \leq \mathrm{Z}(K)$ and $K=H * C$, where $C=\mathrm{C}_{K}(H)$ and $H \cap C=\left\langle h^{p}\right\rangle \geq\langle z\rangle$. On the other hand, $K / H \cong C /\left\langle h^{p}\right\rangle$ is cyclic and so $C$ is abelian of rank 2 (because $\Omega_{1}(C)=U$ ), $C=\mathrm{Z}(K)$ and $K_{1}=\langle h\rangle C$ is an abelian subgroup of index 2 in $K$ with $\Omega_{1}\left(K_{1}\right)=U$.

No element in $U-\langle z\rangle$ is a $p$-th power of an element in $G$. Indeed, if there is $x \in G$ such that $x^{p} \in U-\langle z\rangle$, then we consider the subgroup $U\langle x\rangle \unlhd G$ of order $p^{3}$. Since $\langle z\rangle \leq \mathrm{Z}(G)$ and $x$ commutes with $x^{p}$, it follows that $U\langle x\rangle$ is abelian of type $\left(p^{2}, p\right)$. But then we get $\mho_{1}(U\langle x\rangle)=\left\langle x^{p}\right\rangle \unlhd G$ and so $U \leq \mathrm{Z}(G)$, a contradiction. 
Since $\Omega_{1}\left(K_{1}\right)=U$ and no element in $U-\langle z\rangle$ is a $p$-th power of an element in $K_{1}$, it follows that we have $K_{1}=\langle k\rangle \times\langle u\rangle$ with $u \in U-\langle z\rangle, o(k) \geq p^{n-1}$ and $\langle k\rangle \geq\langle z\rangle$. Note that $\mho_{1}\left(K_{1}\right)=\left\langle k^{p}\right\rangle \leq \mathrm{Z}(K)$ and so $\left\langle k^{p}\right\rangle \times\langle u\rangle \leq \mathrm{Z}(K)$. Suppose that $K>L$ in which case we have $o(k) \geq p^{n}$. But then we get

$$
\Omega_{n-1}\left(K_{1}\right) \leq\left\langle k^{p}\right\rangle \times\langle u\rangle \leq \mathrm{Z}(K)
$$

and since $h \in \Omega_{n-1}\left(K_{1}\right)$, we get $h \in \mathrm{Z}(K)$, a contradiction.

We have proved that we have $K=L$. Since $\langle h\rangle \unlhd G$, we get

$$
[G,\langle h\rangle] \leq\langle h\rangle \cap G^{\prime}=\langle h\rangle \cap U=\langle z\rangle \text { and so }[G,\langle h\rangle]=\langle z\rangle .
$$

It follows that $\mathrm{C}_{G}(h)$ covers $G / K$ and $\mathrm{C}_{K}(h)=\langle h\rangle U$. Hence, if $g \in \mathrm{C}_{G}(h)-$ $K$, then we have $g^{p} \in\langle h\rangle U$ and note that $\left|\mathrm{C}_{G}(h):\langle h\rangle\right|=p^{2}$. Thus, if $g^{p} \in(\langle h\rangle U)-\langle h\rangle$, then $\mathrm{C}_{G}(h)$ would be abelian and $\mathrm{C}_{G}(U) \geq\left\langle\mathrm{C}_{G}(h), t\right\rangle=G$, a contradiction. We have proved that $g^{p} \in\langle h\rangle$ and this gives that either $o(g)=p^{n}$ in which case we may set $g^{p}=h$ or we may assume that $o(g)=p$.

First assume that $p>2$. Assume in addition that $g^{p}=h$. We have $[g, t]=u$ with some $u \in U-\langle z\rangle$ and $u^{g}=u z$, where $\left\langle g^{p^{n-1}}\right\rangle=\langle z\rangle \leq \mathrm{Z}(G)$. It follows that

$$
\left[g^{2}, t\right]=[g, t]^{g}[g, t]=(u z) u=u^{2} z
$$

and we claim that we have $\left[g^{i}, t\right]=u^{i} z^{\left(\begin{array}{c}i \\ 2\end{array}\right)}$ for all $i \geq 2$. Indeed, we get by induction:

$$
\begin{aligned}
& =\left[g^{i} g, t\right]=\left[g^{i}, t\right]^{g}[g, t]=\left(u^{i} z^{\left(\begin{array}{c}
i \\
2
\end{array}\right)}\right)^{g} u=(u z)^{i} z^{\left(\begin{array}{c}
i \\
2
\end{array}\right)} u \\
& =u^{i+1}\left(z^{i+\left(\begin{array}{c}
i \\
2
\end{array}\right)}\right)=u^{i+1} z^{\left(\begin{array}{c}
i+1 \\
2
\end{array}\right)} .
\end{aligned}
$$

This gives

$$
[h, t]=\left[g^{p}, t\right]=u^{p} z^{\left(\begin{array}{c}
p \\
2
\end{array}\right)}=1
$$

which is a contradiction.

We may assume in case $p>2$ that $o(g)=p$, where $[g, h]=1, h^{p^{n-2}}=z$, $n \geq 3$, and $z \in \mathrm{Z}(G)$. We may choose a suitable power $t^{j}$ in $\langle t\rangle, j \not \equiv 0(\bmod$ $p)$, so that we can set from the start that $[h, t]=z$. Then we have $[g, t]=u$ for some $u \in U-\langle z\rangle$ and we have $[g, u]=z^{i}$ with some $i \not \equiv 0(\bmod p)$. We note that

$$
H^{*}=\langle g\rangle \times\langle h\rangle \cong \mathrm{C}_{p} \times \mathrm{C}_{p^{n-1}}, n \geq 3,
$$

is a maximal non-normal subgroup in $G$ since $\left|G: H^{*}\right|=p^{2}$ and $[g, t]=u \notin$ $H^{*}$. Since $\Omega_{1}\left(H^{*}\right) U=\langle g, z\rangle U \cong \mathrm{S}\left(p^{3}\right)$, we are in case (b2) of Proposition 3 with respect to $H^{*}$. But this was excluded by our assumptions.

We have proved that we must have $p=2$. Assume in addition that $o(g)=2$. Then we have $\langle t, g\rangle \cong \mathrm{D}_{8}$ and $[h, t]=z \notin\langle t, g\rangle$ and so $\langle t, g\rangle$ is a non-normal subgroup isomorphic to $\mathrm{D}_{8}$, contrary to our assumptions. Thus we have in this case $g^{2}=h$. Also we have

$$
o(g)=2^{n}, n \geq 4,[g, t]=u \in U-\langle z\rangle, z=g^{2^{n-1}},[g, u]=z
$$


so that

$$
\langle g, u\rangle \cong \mathrm{M}_{2^{n+1}}
$$

is of index 2 in $G$. Also, $\langle h, t\rangle=\left\langle g^{2}, t\right\rangle \cong \mathrm{M}_{2^{n}}$ and $\langle h, t\rangle$ is not normal in $G$ since $[g, t]=u$. We have obtained the groups $G$ stated in our proposition.

We check that there are no involutions in $G-K$, where $K=L=\left\langle g^{2}, t\right\rangle \times$ $\langle u\rangle$ and so we have $\Omega_{1}(G)=\langle u, z, t\rangle \cong \mathrm{E}_{8}$. Indeed, suppose that $g h^{i} u^{j} t^{k}$ is an element in $G-K$, where $g^{2}=h, i$ is any integer and $j, k \in\{0,1\}$. Then we get

$$
x=\left(g h^{i} u^{j} t^{k}\right)^{2}=h^{2 i+1} u^{k} z^{j+i k} \text { and so }\langle x\rangle \geq\langle z\rangle .
$$

If $x=1$, then $k=0$ and so $h^{2 i+1} z^{j}=1$, a contradiction.

Conversely, let $X$ be any noncyclic and non-normal subgroup in $G$ of order $\geq 2^{3}$ containing more than one involution. Then we may assume (up to conjugacy in $G$ ) that $t \in X$ and so $\Omega_{1}(X)=\left\langle t, u^{\prime}\right\rangle$ with some involution $u^{\prime} \in U$. If $X \not \leq K$, then by the above calculation we see that $X$ contains $z$ and so we have $\Omega_{1}(X)=\langle t, z\rangle$. But then for an element $x \in X-K$, we have $[x, t] \in U-\langle z\rangle$ and so in this case $X \geq G^{\prime}=\langle u, z\rangle$, a contradiction. Hence we have $X \leq K$. Note that $\langle h\rangle \unlhd G$ and $\langle h u\rangle \unlhd G$. Since $|X| \geq 2^{3}$, it follows that $X \cap\langle h\rangle \neq\{1\}$ and so $z \in X$ and $\Omega_{1}(X)=\langle t, z\rangle$. Hence we have

$$
X=\langle t\rangle(X \cap\langle h\rangle) \text { or } X=\langle t\rangle(X \cap\langle h u\rangle) .
$$

But both $X \cap\langle h\rangle$ and $X \cap\langle h u\rangle$ are normal in $G$ and we are done. Our group $G$ satisfies the condition (*).

Proposition 4.10. Suppose that we have the case (b1) of Proposition 3, where $H \cong \mathrm{M}_{p^{3}}, p>2$, and $G$ is of class 2 . Then we have the following possibilities:

(a) $G$ is a splitting extension of a cyclic normal subgroup $\langle g\rangle \cong \mathrm{C}_{p^{m}}$, $m \geq 3$, by

$$
\mathrm{M}_{p^{3}} \cong\left\langle h, t \mid h^{p^{2}}=t^{p}=1,[h, t]=h^{p}=z\right\rangle,
$$

where $[g, h]=1$ and $[g, t]=u$ with $\langle u\rangle=\Omega_{1}(\langle g\rangle)$.

We have

$|G|=p^{m+3}, m \geq 3, \mathrm{E}_{p^{2}} \cong G^{\prime}=\langle u, z\rangle, \mathrm{Z}(G)=\left\langle g^{p}\right\rangle \times\langle z\rangle \cong \mathrm{C}_{p^{m-1}} \times \mathrm{C}_{p}$,

$\langle g, h\rangle \cong \mathrm{C}_{p^{m}} \times \mathrm{C}_{p^{2}}$ is a unique abelian maximal subgroup of $G$,

$$
\Omega_{1}(G)=\langle u, z, t\rangle \cong \mathrm{E}_{p^{3}}
$$

and

$$
\langle h, t\rangle \cong \mathrm{M}_{p^{3}} \text { and }\langle g, t\rangle \cong \mathrm{M}_{p^{m+1}}
$$

are non-normal subgroups in $G$ with $\langle h\rangle \unlhd G$ and $\langle g\rangle \unlhd G$. 
(b) $G=(\langle g\rangle \times\langle h\rangle)\langle t\rangle$, where $\langle g\rangle \cong\langle h\rangle \cong \mathrm{C}_{p^{2}}, g^{p}=u, h^{p}=z$, $t$ centralizes $\langle u, z\rangle$

$$
[h, t]=z,[g, t]=u^{i} z^{j}, i \not \equiv 0(\bmod p) .
$$

Here $G$ is a special group of order $p^{5}$ with

$$
\mathrm{E}_{p^{2}} \cong G^{\prime}=\langle u, z\rangle, \Omega_{1}(G)=\langle u, z, t\rangle \cong \mathrm{E}_{p^{3}}
$$

and $\langle h, t\rangle \cong \mathrm{M}_{p^{3}}$ is non-normal in $G$ with $\langle h\rangle \unlhd G$.

Conversely, all groups in (a) and (b) satisfy our assumption (*).

Proof. By Proposition 6, $H$ possesses a $G$-invariant cyclic subgroup $\langle h\rangle \cong \mathrm{C}_{p^{2}}$ and then we may set:

$$
H=\left\langle h, t \mid h^{p^{2}}=t^{p}=1,[h, t]=h^{p}=z\right\rangle .
$$

Since $K /\langle t, z\rangle$ is abelian, we have $K^{\prime}=H^{\prime}=\langle z\rangle$ and so $K=H * C$ with $H \cap C=\langle z\rangle$, where $C=\mathrm{C}_{K}(H)$. Also, $K / H \cong C /\langle z\rangle$ is cyclic of order $\geq p$ and so $C$ and $C_{1}=\langle h\rangle C$ are abelian, where $\Omega_{1}\left(C_{1}\right)=U=G^{\prime} \leq \mathrm{Z}(G)$ and $\mho_{1}(G) \leq \mathrm{Z}(G)$.

Since $[G,\langle h\rangle]=\langle z\rangle$, we have $G=\langle t\rangle \mathrm{C}_{G}(h)$. Set $S=U \times\langle t\rangle \cong \mathrm{E}_{p^{3}}$ and because $\left|G: \mathrm{C}_{G}(t)\right|=p^{2}$, all $p^{2}$ subgroups of order $p$ in $(S-U) \cup\{1\}$ form a single conjugate class in $G$. We have $\Omega_{1}(K)=S$ and we have in fact $\Omega_{1}(G)=S$. Indeed, if $g$ is an element of order $p$ in $G-K$, then we have

$$
\langle g, t\rangle \cong \mathrm{S}\left(p^{3}\right) \text { with } u^{\prime}=[g, t] \in U-\langle z\rangle .
$$

Because $\langle g, t\rangle \cap K=\left\langle t, u^{\prime}\right\rangle \cong \mathrm{E}_{p^{2}}$, we have $z \notin\langle g, t\rangle$. But $[h, t]=z$ and so $\langle g, t\rangle$ is not normal in $G$, contrary to Proposition 1.

(i) First assume that $G / L$ is cyclic of order $\geq p^{2}$, where $L=H U$. Let $g \in \mathrm{C}_{G}(h)-K$ so that $\langle g\rangle$ covers $G / L$ and $\left\langle g^{p}\right\rangle \leq \mathrm{Z}(G)$ covers $K / H$ (which is cyclic of order $\left.\geq p^{2}\right)$. Hence we have $\Omega_{1}\langle g\rangle=\langle u\rangle$, where $o(g)=p^{m}, m \geq 3$, $u \in U-\langle z\rangle$ and $[g, t]=u z^{i}$ for some integer $i(\bmod p)$. We replace $g$ with $g^{\prime}=h^{-i} g \in \mathrm{C}_{G}(h)-K$ so that we have

$$
\left[g^{\prime}, t\right]=\left[h^{-i} g, t\right]=z^{-i}\left(u z^{i}\right)=u, \text { where }\left(g^{\prime}\right)^{p^{m-1}}=\left(h^{-i} g\right)^{p^{m-1}}=g^{p^{m-1}}
$$

with $\left\langle g^{p^{m-1}}\right\rangle=\langle u\rangle$. Thus, we may assume from the start that $[g, t]=u$ and so $\langle g, t\rangle \cong \mathrm{M}_{p^{m+1}}$ with $\langle g\rangle \unlhd G$. But $[h, t]=z=h^{p}$ and so $z \notin\langle g, t\rangle$ and therefore $\langle g, t\rangle$ is a maximal non-normal subgroup in $G$. Our group $G$ is a splitting extension of $\langle g\rangle$ by $\langle h, t\rangle$ and so we have obtained the groups stated in part (a) of our proposition. We check that

$$
\Omega_{1}(G)=S=\langle u, z, t\rangle \cong \mathrm{E}_{p^{3}} .
$$

Indeed, let $1 \neq t^{\prime} \in\langle t\rangle$ and suppose that $x=t^{\prime} g^{r} h^{s}$ ( $r, s$ are any integers) is an element of order $p$ in $G-\langle g, h\rangle$. Then we have

$$
1=\left(t^{\prime}\left(g^{r} h^{s}\right)\right)^{p}=\left(t^{\prime}\right)^{p} g^{p r} h^{p s}\left[g^{r} h^{s}, t^{\prime}\right]^{\left(\begin{array}{c}
p \\
2
\end{array}\right)}=g^{p r} h^{p s} .
$$

Hence $r \equiv 0\left(\bmod p^{m-1}\right), s \equiv 0(\bmod p)$ and so we get $x \in S$. 
Conversely, let $X$ be a noncyclic and non-normal subgroup of order $\geq p^{3}$ in $G$. We may assume (up to conjugacy in $G$ ) that $t \in X$ and so $\Omega_{1}(X)=$ $\left\langle t, u^{\prime}\right\rangle \cong \mathrm{E}_{p^{2}}$, where $u^{\prime}$ is an element of order $p$ in $U$. Set $X_{0}=X \cap\langle g, h\rangle$ so that $X_{0}$ is cyclic and $\mathrm{N}_{G}\left(X_{0}\right) \geq\langle g, h\rangle\langle t\rangle=G$. Our condition $(*)$ is satisfied.

(ii) Assume that either $K=L$ or $K>L$ but $G / L$ is noncyclic so that $G / K$ splits over $K / L$. In any case we have $G=K G_{0}$ with $K \cap G_{0}=L$ and $\left|G_{0}: L\right|=p$. We have $\mathrm{C}_{G_{0}}(h)=(\langle h\rangle U)\langle g\rangle$ for some $g \in G_{0}-K$. Since there are no elements of order $p$ in $G_{0}-K$, we have $o(g) \geq p^{2}$ and so $g^{p} \in \mathrm{Z}(G) \cap L$ implies that $1 \neq g^{p} \in U$. If $g^{p} \in\langle z\rangle$, then $\langle g, h\rangle$ would contain elements of order $p$ in $G_{0}-K$, a contradiction. Hence we must have $g^{p}=u \in U-\langle z\rangle$.

Suppose that $K>L$. Then there is an element $a \in C-U$ of order $p^{2}$ so that $a^{p}=u^{\prime} \in U-\langle z\rangle$. Considering the subgroup $\langle h\rangle \times\langle g\rangle \cong \mathrm{C}_{p^{2}} \times \mathrm{C}_{p^{2}}$, each element in $\mho_{1}(\langle g, h\rangle)=\langle u, z\rangle$ is a $p$-th power of an element in $\langle g, h\rangle$. Thus, there is $y \in\langle g, h\rangle-K$ such that $y^{p}=\left(u^{\prime}\right)^{-1}$. But then we get:

$$
(a y)^{p}=a^{p} y^{p}[y, a]^{\left(\begin{array}{c}
p \\
2
\end{array}\right)}=u^{\prime}\left(u^{\prime}\right)^{-1}=1,
$$

and so $a y$ is an element of order $p$ in $G-K$, a contradiction. Hence we have $K=L$. In this case we have $[g, t]=u^{i} z^{j}$ with $i \not \equiv 0(\bmod p)$ and so we have obtained a special group of order $p^{5}$ stated in part (b) of our proposition. We check that

$$
\Omega_{1}(G)=S=\langle u, z, t\rangle \cong \mathrm{E}_{p^{3}} .
$$

Indeed, let $1 \neq t^{\prime} \in\langle t\rangle$ and suppose that $x=t^{\prime} g^{r} h^{s}$ ( $r, s$ are any integers) is an element of order $p$ in $G-\langle g, h\rangle$. Then we have

$$
1=\left(t^{\prime}\left(g^{r} h^{s}\right)\right)^{p}=\left(t^{\prime}\right)^{p} g^{p r} h^{p s}\left[g^{r} h^{s}, t^{\prime}\right]^{\left(\begin{array}{c}
p \\
2
\end{array}\right)}=g^{p r} h^{p s} .
$$

Hence $r \equiv 0(\bmod p), s \equiv 0(\bmod p)$ and so we get $x \in S$.

Conversely, let $X$ be a noncyclic and non-normal subgroup of order $p^{3}$ in $G$. We may assume (up to conjugacy in $G$ ) that $t \in X$ and so $\Omega_{1}(X)=$ $\left\langle t, u^{\prime}\right\rangle \cong \mathrm{E}_{p^{2}}$, where $u^{\prime}$ is an element of order $p$ in $U$. Set $X_{0}=X \cap\langle g, h\rangle$ so that $X_{0}$ is cyclic of order $p^{2}$ and $\mathrm{N}_{G}\left(X_{0}\right) \geq\langle g, h\rangle\langle t\rangle=G$. Our assumption $(*)$ is satisfied.

Proposition 4.11. Suppose that we have the case (b1) of Proposition 3, where $H \cong \mathrm{M}_{p^{n}}, n \geq 4$, is a non-normal subgroup of maximal possible order in $G$ ( which is isomorphic to some $\mathrm{M}_{p^{m}}, m \geq 4$ ), $G$ is of class 2 and assume that $G$ does not have non-normal subgroups isomorphic to $\mathrm{D}_{8}$ or $\mathrm{M}_{p^{3}}$ with $p>2$. Then we have the following possibilities:

(a) $G=(\langle h\rangle \times\langle g\rangle)\langle t\rangle$, where

$$
\langle h\rangle \cong \mathrm{C}_{p^{n-1}}, n \geq 4,\langle g\rangle \cong \mathrm{C}_{p^{m}}, m \geq 3,\langle t\rangle \cong \mathrm{C}_{p},
$$

$[h, t]=z$ with $\langle z\rangle=\Omega_{1}(\langle h\rangle),[g, t]=z^{i} u$ with $\langle u\rangle=\Omega_{1}(\langle g\rangle), i$ integer, and $t$ centralizes $\langle u, z\rangle$. 
Here we have $|G|=p^{m+n}, m \geq 3, n \geq 4$,

$$
\mathrm{E}_{p^{2}} \cong G^{\prime}=\langle u, z\rangle \leq \mathrm{Z}(G), \Omega_{1}(G)=\langle u, z, t\rangle \cong \mathrm{E}_{p^{3}},
$$

$\langle g, h\rangle \cong \mathrm{C}_{p^{m}} \times \mathrm{C}_{p^{n-1}}$ is a unique abelian maximal subgroup of $G$ and $\langle h, t\rangle \cong \mathrm{M}_{p^{n}}$ is non-normal in $G$ with $\langle h\rangle \unlhd G$.

(b) $G=(\langle k\rangle \times\langle g\rangle)\langle t\rangle$, where

$$
\langle g\rangle \cong \mathrm{C}_{p^{n}}, n \geq 4,\langle k\rangle \cong \mathrm{C}_{p^{m}}, 2 \leq m \leq n-2,\langle t\rangle \cong \mathrm{C}_{p},
$$$$
[k, t]=z \text { with }\langle z\rangle=\Omega_{1}(\langle g\rangle),[g, t]=u \text { with }\langle u\rangle=\Omega_{1}(\langle k\rangle),
$$

and $t$ centralizes $\langle u, z\rangle$.

Here we have $|G|=p^{m+n+1}, n \geq 4,2 \leq m \leq n-2$,

$$
\mathrm{E}_{p^{2}} \cong G^{\prime}=\langle u, z\rangle \leq \mathrm{Z}(G), \Omega_{1}(G)=\langle u, z, t\rangle \cong \mathrm{E}_{p^{3}},
$$

$\langle g, k\rangle \cong \mathrm{C}_{p^{n}} \times \mathrm{C}_{p^{m}}$ is a unique abelian maximal subgroup of $G$ and $\left\langle k g^{p}, t\right\rangle \cong \mathrm{M}_{p^{n}}$ is non-normal in $G$ with $\left\langle k g^{p}\right\rangle \unlhd G$.

Conversely, all groups in (a) and (b) satisfy our assumption $(*)$.

Proof. By Proposition $4, G^{\prime} \leq U$ and so $G^{\prime}=U \leq \mathrm{Z}(G)$ and $\mho_{1}(G) \leq$ $\mathrm{Z}(G)$. Also, Proposition 7 implies that $K / \Omega_{1}(H)$ is abelian and so $K / H$ is cyclic (by Proposition 2), where $\Omega_{1}(H) \cong \mathrm{E}_{p^{2}}$ and therefore we have $K^{\prime}=$ $H^{\prime}=\langle z\rangle \leq \mathrm{Z}(G)$. By Proposition $2, K / H$ is cyclic of order $\geq p$. Finally, Proposition 3 also implies that $U=\Omega_{1}(\mathrm{Z}(L))$, where $L=H U \unlhd G$. By Proposition $6, H$ possesses a $G$-invariant cyclic subgroup $\langle h\rangle$ of index $p$ and there is an element $t$ of order $p$ in $H-\langle h\rangle$ so that $\langle[h, t]\rangle=\langle z\rangle$. For any $g \in G-K$, we have $t^{g}=t u^{\prime}$ for some $u^{\prime} \in U-\langle z\rangle$, where $G / K \cong \mathrm{C}_{p}$, $S=\langle t\rangle U \cong \mathrm{E}_{p^{3}}$ is normal in $G$ and $S=\Omega_{1}(K)$. It follows that all $p^{2}$ subgroups of order $p$ contained in $(S-U) \cup\{1\}$ form a single conjugate class in $G$.

Since $K^{\prime}=H^{\prime}$, we get $K=H * C$, where $C=\mathrm{C}_{K}(H)$ and $H \cap C=$ $\left\langle h^{p}\right\rangle \geq\langle z\rangle$. On the other hand, $K / H \cong C /\left\langle h^{p}\right\rangle$ is cyclic and so $C$ is abelian of rank 2 (because $\left.\Omega_{1}(C)=U\right), C=\mathrm{Z}(K)$ and $K_{1}=\langle h\rangle C$ is an abelian subgroup of index 2 in $K$ with $\Omega_{1}\left(K_{1}\right)=U$. Since $\langle h\rangle \unlhd G$, we get

$$
[G,\langle h\rangle] \leq\langle h\rangle \cap G^{\prime}=\langle h\rangle \cap U=\langle z\rangle \text { and so }[G,\langle h\rangle]=\langle z\rangle .
$$

It follows that $G=\langle t\rangle \mathrm{C}_{G}(h)$.

It is easy to see that there are no elements of order $p$ in $G-K$. Indeed, suppose that there is an element $i$ of order $p$ in $G-K$. Since $[i, t]=u \in U-\langle z\rangle$, we get that $D=\langle i, t\rangle$ is isomorphic to $\mathrm{D}_{8}$ in case $p=2$ and $D$ is isomorphic to $\mathrm{S}\left(p^{3}\right)$ in case $p>2$. On the other hand, $D \cap K=\langle t, u\rangle \cong \mathrm{E}_{p^{2}}$ and we have $[h, t]=z$, where $\langle z\rangle=\Omega_{1}(\langle h\rangle)$. Hence $D$ is not normal in $G$. But the case $D \cong \mathrm{D}_{8}$ is excluded by our assumptions and the case case $D \cong \mathrm{S}\left(p^{3}\right)$ is not possible by Proposition 1.

First we consider the case, where $G / L$ (being abelian as a factor-group of the abelian group $G / U)$ is not cyclic of order $\geq p^{2}$. Hence we have either 
$G / L \cong \mathrm{C}_{p}$ (i.e., $\left.K=L\right)$ or $G / L$ is abelian of type $\left(p^{r}, p\right), r \geq 1$ (noting that $K / H$ is cyclic and so $K / L$ is cyclic). In any case, $G / L$ splits over $K / L$ and so $G$ has a normal subgroup $G_{0}$ such that $G=K G_{0}$ with $K \cap G_{0}=L$ and $\left|G_{0}: L\right|=p$. Since $\left[G_{0},\langle h\rangle\right]=\langle z\rangle$, it follows that $\mathrm{C}_{G_{0}}(h)$ covers $G_{0} / L$, where $\mathrm{C}_{L}(h)=\langle h\rangle U$ and so $\mathrm{C}_{G_{0}}(h)$ is abelian of rank 2 with $\Omega_{1}\left(\mathrm{C}_{G_{0}}(h)\right)=U$ (noting that there are no elements of order $p$ in $G_{0}-L$ ). If $\mathrm{C}_{G_{0}}(h)$ is abelian of type $\left(p^{n}, p\right)$, then there is an element $g_{1} \in \mathrm{C}_{G_{0}}(h)-(\langle h\rangle U)$ such that $\left(g_{1}\right)^{p}=h u^{i}(0 \leq i \leq p-1)$, where $u \in U-\langle z\rangle$. But then $\left(g_{1}\right)^{p}=h u^{i} \in \mathrm{Z}(G)$ and so $h \in \mathrm{Z}(G)$, a contradiction. Hence $\mathrm{C}_{G_{0}}(h)$ is of type $\left(p^{n-1}, p^{2}\right)$ and therefore there is an element $g \in \mathrm{C}_{G_{0}}(h)-K$ such that $g^{p}=u \in U-\langle z\rangle$. We may assume that $[t, g]=u z^{i}(0 \leq i \leq p-1)$ (by replacing $t$ with a suitable power $\neq 1$ of $t$, if necessary) and then we choose an element $h^{\prime} \in\left\langle h^{p}\right\rangle$ such that $\left(h^{\prime}\right)^{p}=z^{i}$ (noting that $o(h)=p^{n-1} \geq p^{3}$ ). Then we take the element $g^{\prime}=h^{\prime} g \in G_{0}-K$ and compute:

$$
\left(g^{\prime}\right)^{p}=\left(h^{\prime}\right)^{p} g^{p}=u z^{i} \text { and }\left[t, g^{\prime}\right]=\left[t, h^{\prime} g\right]=[t, g]=u z^{i} .
$$

Hence, in case $p=2$ we have $\left\langle g^{\prime}, t\right\rangle \cong \mathrm{D}_{8}$ and then $g^{\prime} t$ is an involution in $G_{0}-K$, a contradiction. If $p>2$, then $\left\langle g^{\prime}, t\right\rangle \cong \mathrm{M}_{p^{3}}$. But we have

$$
\left\langle g^{\prime}, t\right\rangle \cap K=\left\langle\left(g^{\prime}\right)^{p}, t\right\rangle \cong \mathrm{E}_{p^{2}} \text { and } 1 \neq[h, t] \in\langle z\rangle \notin\left\langle g^{\prime}, t\right\rangle .
$$

Thus, $\left\langle g^{\prime}, t\right\rangle$ is a non-normal subgroup in $G$ isomorphic to $\mathrm{M}_{p^{3}}, p>2$, which was excluded by our assumptions.

We have proved that $G / L$ must be cyclic of order $\geq p^{2}$. Let $g \in \mathrm{C}_{G}(h)-K$ so that $\langle g\rangle$ covers $G / L$ and we have $g^{p} \in \mathrm{Z}(G)$. But $K / H$ is cyclic of order $\geq p^{2}$ and so $\left\langle g^{p}\right\rangle$ (covering $\left.K / L\right)$ covers $K / H$. Hence $\langle g\rangle$ covers $\mathrm{C}_{G}(h) /\langle h\rangle$ and so $A=\mathrm{C}_{G}(h)$ is abelian of rank 2 because $\Omega_{1}(A)=U$. We also have $|A /\langle h\rangle| \geq p^{3}$.

(i) First assume that $A$ splits over $\langle h\rangle$. Then we may set $A=\langle h\rangle \times\langle g\rangle$ with $o(g)=p^{m}, m \geq 3$, and $\Omega_{1}(\langle g\rangle)=\langle u\rangle$. We have $[h, t]=z$ with $\Omega_{1}(\langle h\rangle)=\langle z\rangle$ and $[g, t]=z^{i} u$, where $i$ is an integer $(\bmod p)$.

We have obtained the groups stated in part (a) of our proposition. Now we check that we have

$$
\Omega_{1}(G)=S=\langle u, z, t\rangle \cong \mathrm{E}_{p^{3}} .
$$

Indeed, let $1 \neq t^{\prime} \in\langle t\rangle$ and let $x=t^{\prime} h^{r} g^{s}$ ( $r, s$ are any integers) be an element of order $p$. Then we get in case $p>2$ :

$$
1=\left(t^{\prime}\left(h^{r} g^{s}\right)\right)^{p}=\left(t^{\prime}\right)^{p} h^{r p} g^{s p}\left[h^{r} g^{s}, t^{\prime}\right]^{\left(\begin{array}{c}
p \\
2
\end{array}\right)}=h^{r p} g^{s p} .
$$

This implies

$$
r \equiv 0\left(\bmod p^{n-2}\right) \text { and } s \equiv 0\left(\bmod p^{m-1}\right) \text { and so } x \in S .
$$

Suppose that $p=2$. Then we have :

$$
1=\left(t\left(h^{r} g^{s}\right)\right)^{2}=t^{2} h^{2 r} g^{2 s}\left[h^{r} g^{s}, t\right]=h^{2 r} g^{2 s} z^{r} z^{i s} u^{s}=\left(h^{2 r} z^{r+i s}\right)\left(g^{2 s} u^{s}\right) .
$$


This implies $r \equiv 0\left(\bmod 2^{n-3}\right)$ and $s \equiv 0\left(\bmod 2^{m-2}\right)$. Since $n \geq 4$ and $m \geq 3$, this gives $z^{r+i s}=u^{s}=1$ and then we get $h^{2 r} g^{2 s}=1$ and therefore $r \equiv 0\left(\bmod 2^{n-2}\right), s \equiv 0\left(\bmod 2^{m-1}\right)$ and $x \in S$.

(ii) Assume that $A$ does not split over $\langle h\rangle$. Then we have for an element $g \in A-K$ the following facts:

$$
A=\langle h\rangle\langle g\rangle,\langle h\rangle \cap\langle g\rangle \geq\langle z\rangle \text { and } o(h)=p^{n-1}<o(g) .
$$

Suppose that $o(g)>p^{n}$. Then we have $o\left(g^{p}\right) \geq p^{n}$ and $g^{p} \in \mathrm{Z}(G)$. In this case we get:

$$
\left(h g^{p}\right)^{p^{n-1}}=g^{p^{n}} \geq\langle z\rangle,\left[t, h g^{p}\right]=[t, h],\langle[t, h]\rangle=\langle z\rangle,[t, g]=u^{\prime} \in U-\langle z\rangle,
$$

and this shows that $\left\langle t, h g^{p}\right\rangle \cong \mathrm{M}_{p^{r}}, r \geq n+1$, is non-normal in $G$, contrary to our maximality assumption.

We have proved that we must have $o(g)=p^{n}$. Also we get:

$$
|A:\langle g\rangle|=|\langle h\rangle:(\langle h\rangle \cap\langle g\rangle)|=p^{m} \text { with } m \leq n-2 \text { since }\langle h\rangle \cap\langle g\rangle \geq\langle z\rangle \text {. }
$$

If $m \leq 1$, then $A=\langle g\rangle U$ and so $\left\langle g^{p}\right\rangle U=A \cap K \leq \mathrm{Z}(G)$, contrary to $h \notin \mathrm{Z}(G)$. Hence we must have $m \geq 2$. Since $\left\langle g^{p}\right\rangle$ (of order $p^{n-1}$ ) splits in $A \cap K$, we get $A \cap K=\langle k\rangle \times\left\langle g^{p}\right\rangle$ and so we have $A=\langle k\rangle \times\langle g\rangle$ with $o(k)=p^{m}, 2 \leq m \leq n-2$. Because $[A \cap K,\langle t\rangle]=\langle z\rangle$, we have $[k, t]=z$, where $\langle z\rangle=\Omega_{1}(\langle g\rangle)$.

Further we have $[g, t]=u z^{i}$ ( $i$ some integer) with $\langle u\rangle=\Omega_{1}(\langle k\rangle)$. We may replace $g$ with $g^{\prime}=k^{-i} g$ so that we have:

$$
\begin{aligned}
& \left(g^{\prime}\right)^{p^{n-1}}=\left(k^{-i} g\right)^{p^{n-1}}=g^{p^{n-1}}, \\
& \left\langle g^{p^{n-1}}\right\rangle=\langle z\rangle, \\
& {\left[g^{\prime}, t\right]=\left[k^{-i} g, t\right]=z^{-i}\left(u z^{i}\right)=u,}
\end{aligned}
$$

and so writing again $g$ instead of $g^{\prime}$, we can assume from the start that $[g, t]=u$. Also we have:

$$
1 \neq\left(k g^{p}\right)^{p^{n-2}}=g^{p^{n-1}} \geq\langle z\rangle, \quad\left[k g^{p}, t\right]=z,[g, t]=u,
$$

and so $\left\langle k g^{p}, t\right\rangle \cong \mathrm{M}_{p^{n}}$ is non-normal in $G$ with $\left\langle k g^{p}\right\rangle \unlhd G$. We have obtained the groups stated in part (b) of our proposition.

Now we check that we have

$$
\Omega_{1}(G)=S=\langle u, z, t\rangle \cong \mathrm{E}_{p^{3}} .
$$

Indeed, let $1 \neq t^{\prime} \in\langle t\rangle$ and let $x=t^{\prime} k^{r} g^{s}$ ( $r, s$ are any integers) be an element of order $p$. Then we get in case $p>2$ :

$$
1=\left(t^{\prime}\left(k^{r} g^{s}\right)\right)^{p}=\left(t^{\prime}\right)^{p} k^{r p} g^{s p}\left[k^{r} g^{s}, t^{\prime}\right]^{\left(\begin{array}{l}
p \\
2
\end{array}\right)}=k^{r p} g^{s p} .
$$

This implies

$$
r \equiv 0\left(\bmod p^{m-1}\right) \text { and } s \equiv 0\left(\bmod p^{n-1}\right) \text { and so } x \in S .
$$


Suppose that $p=2$. Then we have :

$$
1=\left(t\left(k^{r} g^{s}\right)\right)^{2}=t^{2} k^{2 r} g^{2 s}\left[k^{r} g^{s}, t\right]=k^{2 r} g^{2 s} z^{r} u^{s}=\left(k^{2 r} u^{s}\right)\left(g^{2 s} z^{r}\right) .
$$

This implies $s \equiv 0\left(\bmod 2^{n-2}\right)$ and so $1=k^{2 r}\left(g^{2 s} z^{r}\right)$ and $r \equiv 0\left(\bmod 2^{m-1}\right)$ which gives $g^{2 s}=1$ and $s \equiv 0\left(\bmod 2^{n-1}\right)$. Hence we get again $x \in S$.

It remains to prove in case of both groups in parts (a) and (b) of our proposition that the assumption $(*)$ is satisfied. Indeed, let $A$ be a unique abelian maximal subgroup of $G$, where $t \in G-A$ ( since $\Omega_{1}(A)=U=G^{\prime}$ ). Let $X$ be a noncyclic and non-normal subgroup of order $\geq p^{3}$ in $G$ which in case $p=2$ has more than one involution. Since $X \nsupseteq G^{\prime}$ and all noncentral subgroups of order $p$ form a single conjugate class in $G$ (with a representative $\langle t\rangle)$, we may assume that $t \in X$. We set $X_{0}=X \cap A$, where $X_{0}$ is cyclic since

$$
\Omega_{1}(X)=\left\langle t, u^{\prime}\right\rangle \text { for some } 1 \neq u^{\prime} \in G^{\prime}=\Omega_{1}(A) .
$$

But then we have $\mathrm{N}_{G}\left(X_{0}\right) \geq\langle A, t\rangle=G$ and we are done. Our proposition is completely proved.

In the next proposition we collect all the remaining $p$-groups satisfying the condition $(*)$.

Proposition 4.12. Suppose that $G$ is a p-group satisfying $(*)$ which is not a 2-group of maximal class, $G$ has no non-normal subgroups isomorphic to $\mathrm{D}_{8}$ or $\mathrm{M}_{p^{n}},\left|G^{\prime}\right|=p^{2}, K / \Omega_{1}(H)$ is abelian for each abelian noncyclic maximal non-normal subgroup $H$ of order $\geq p^{3}$ in $G$, and $G$ has no nonnormal abelian subgroups which lead to the case (b2) of Proposition 3. Then we have the following possibilities.

(a) G has a maximal subgroup

$\mathrm{M}_{p^{s+2}} \cong\left\langle g, u \mid g^{p^{s+1}}=u^{p}=1,[u, g]=z,\langle z\rangle=\Omega_{1}(\langle g\rangle)\right\rangle, p>2, s \geq 2$,

$G=\langle g, u\rangle\langle t\rangle$, where $o(t)=p,[g, t]=u$ and $[u, t]=1$.

These groups are actually $\mathrm{A}_{2}$-groups defined in Proposition 71.3(i) in [2], where $\left\langle g^{p}, t\right\rangle \cong \mathrm{C}_{p^{s}} \times \mathrm{C}_{p}$ is non-normal in $G$ with $\left\langle g^{p}\right\rangle \unlhd G$.

(b) $G$ is a special group of order $2^{5}$ with a unique abelian maximal subgroup

$$
K=\langle h\rangle \times\langle u\rangle \times\langle t\rangle,\langle h\rangle \cong \mathrm{C}_{4}, h^{2}=z,\langle u\rangle \cong\langle t\rangle \cong \mathrm{C}_{2},
$$

and $G=K\langle g\rangle$, where $g^{2}=z,[g, h]=z,[g, u]=1,[g, t]=u$.

Here we have $G^{\prime}=\langle u, z\rangle \cong \mathrm{E}_{4}, \Omega_{1}(G)=\langle u, z, t\rangle \cong \mathrm{E}_{8}$ and $\langle h, t\rangle \cong$ $\mathrm{C}_{4} \times \mathrm{C}_{2}$ is a non-normal subgroup in $G$ with $\langle h\rangle \unlhd G$.

(c) $G$ has a maximal subgroup

$$
\left\langle h, g \mid h^{p^{s}}=g^{p^{r}}=1, h^{p^{s-1}}=z,[g, h]=z\right\rangle, s \geq 4,3 \leq r<s
$$

and

$$
G=\langle h, g\rangle\langle t\rangle \text { with } t^{p}=1,[h, t]=1,[g, t]=u z^{i}, i \not \equiv 0(\bmod p),
$$

$$
\langle u\rangle=\Omega_{1}(\langle g\rangle),[u, t]=1 .
$$


We have $|G|=p^{r+s+1}, \mathrm{E}_{p^{2}} \cong G^{\prime}=\langle u, z\rangle \leq \mathrm{Z}(G), \Omega_{1}(G)=$ $\langle u, z, t\rangle \cong \mathrm{E}_{p^{3}}$,

$$
K=\left\langle t, h, g^{p}\right\rangle \cong \mathrm{C}_{p} \times \mathrm{C}_{p^{s}} \times \mathrm{C}_{p^{r-1}}
$$

is a unique abelian maximal subgroup in $G$ and

$$
\langle h, t\rangle \cong \mathrm{C}_{p^{s}} \times \mathrm{C}_{p}
$$

is an abelian maximal non-normal subgroup in $G$ with $\langle h\rangle \unlhd G$.

(d) $G$ is a 2-group which possesses a normal subgroup $G_{0}=L\langle g\rangle$, where

$$
\begin{gathered}
L=\langle h\rangle \times\langle u\rangle \times\langle t\rangle,\langle h\rangle \cong \mathrm{C}_{4}, h^{2}=z,\langle u\rangle \cong\langle t\rangle \cong \mathrm{C}_{2}, \\
g^{2}=z,[g, h]=z,[g, u]=1,[g, t]=u,
\end{gathered}
$$

which is a special group of order $2^{5}$ with $G_{0}^{\prime}=\langle u, z\rangle \cong \mathrm{E}_{4}$. Then we have the following possibilities for $G=G_{0}\langle k\rangle$ :

(d1) $k^{4}=u,[k, g]=1,[k, t]=z,[k, h]=z$, and here we have $|G|=2^{7}, \exp (G)=8$ and $\mathrm{Z}(G)=G^{\prime}\left\langle k^{2}\right\rangle \cong \mathrm{C}_{4} \times \mathrm{C}_{2}$.

(d2) $k^{2}=u,[k, g]=[k, t]=[k, h]=1$, and here we have $|G|=2^{6}$, $\exp (G)=4$ and $\mathrm{Z}(G)=G^{\prime}\langle k\rangle \cong \mathrm{C}_{4} \times \mathrm{C}_{2}$.

(d3) $k^{2}=u z,[k, g]=[k, h]=1,[k, t]=z$ and here $G$ is a special group of order $2^{6}$ with $\mathrm{Z}(G)=\langle u, z\rangle \cong \mathrm{E}_{4}$.

In all three cases we have $\mathrm{E}_{4} \cong G^{\prime}=\langle u, z\rangle \leq \mathrm{Z}(G), \Omega_{1}(G)=G^{\prime} \times\langle t\rangle \cong$ $\mathrm{E}_{8}$ and $\langle h, t\rangle \cong \mathrm{C}_{4} \times \mathrm{C}_{2}$ is an abelian maximal non-normal subgroup in $G$ with $\langle h\rangle \unlhd G$.

(e) We have $G=(\langle a\rangle \times\langle b\rangle)\langle t\rangle$, where

$$
\langle a\rangle \cong \mathrm{C}_{p^{s+1}},\langle b\rangle \cong \mathrm{C}_{p^{r}},\langle t\rangle \cong \mathrm{C}_{p}, s \geq 2,2 \leq r \leq s+1,
$$

$z=a^{p^{s}}, u=b^{p^{r-1}},[b, t]=z,[a, t]=u^{i} z^{j}, i \neq \equiv(\bmod p),[z, t]=[u, t]=1$.

If $r=s+1$, then $j \not \equiv \xi-i \xi^{-1}(\bmod p)$ for all integers $\xi \not \equiv 0(\bmod p)$.

We have here $|G|=p^{r+s+2}, G^{\prime}=\langle u, z\rangle \cong \mathrm{E}_{p^{2}}, \Omega_{1}(G)=G^{\prime} \times\langle t\rangle \cong$ $\mathrm{E}_{p^{3}}, G$ is of class 2 with

$$
\Phi(G)=\mho_{1}(G)=\mathrm{Z}(G)=\left\langle a^{p}\right\rangle \times\left\langle b^{p}\right\rangle \cong \mathrm{C}_{p^{s}} \times \mathrm{C}_{p^{r-1}} .
$$

Finally, $\left\langle a^{p}\right\rangle \times\langle t\rangle \cong \mathrm{C}_{p^{s}} \times \mathrm{C}_{p}$ is a maximal non-normal subgroup of $G$ with $\left\langle a^{p}\right\rangle \unlhd G$.

Conversely, all the above groups from (a) to (e) satisfy our condition (*).

Proof. Let $G$ be a $p$-group satisfying all assumptions of this proposition. Let $H$ be a maximal non-normal subgroup of a maximal possible order in $G$ which is abelian of type $\left(p^{s}, p\right), s \geq 2$.

Set $U_{0}=U \cap H=\langle z\rangle \leq \mathrm{Z}(\bar{G})$ and $H_{0}=\Omega_{1}(H)=\langle t, z\rangle$ so that $S=$ $H_{0} U \cong \mathrm{E}_{p^{3}}, S=\Omega_{1}(K)=\Omega_{1}(L)$ and $L$ is abelian with $\mho_{1}(L)=\mho_{1}(H) \geq$ $U_{0}$. Also, $K / H_{0}$ is abelian and since $G^{\prime} \leq U$ (Proposition 4 ), we have here $G^{\prime}=U$ (see Proposition 3(b1)) because by our assumption $\left|G^{\prime}\right|=p^{2}$ and so $K^{\prime} \leq\langle z\rangle$ and $G / L$ is abelian. By Proposition $6, H$ possesses a $G$-invariant 
cyclic subgroup $\langle h\rangle \cong \mathrm{C}_{p^{s}}$ which contains $z$ and so we have $H=\langle h\rangle \times\langle t\rangle$. Also, $\mathrm{N}_{G}\left(H_{0}\right)=K$ and by Proposition $2, K / H$ is cyclic of order $\geq p$. By Proposition 3, for each $g \in G-K$, we have $[g, t]=u \in U-\langle z\rangle$, where $|G / K|=p$. We shall use all these facts in the proof of this proposition.

First we prove that there are no elements of order $p$ in $G-K$ and so we have $\Omega_{1}(G)=S=G^{\prime} \times\langle t\rangle \cong \mathrm{E}_{p^{3}}$. Indeed, let $i$ be an element of order $p$ in $G-K$. We have $[i, t]=u^{\prime} \in U-\langle z\rangle$ and so $\langle h, i\rangle$ is not normal in $G$ because $\langle h, i\rangle \cap K=\langle h\rangle$. It follows that

$$
H^{*}=\langle h, i\rangle=\langle h\rangle \times\langle i\rangle
$$

is abelian and the fact that $\left|H^{*}\right|=|H|$ together with the maximality of $|H|$ implies that $H^{*}$ is another maximal non-normal subgroup in $G$ of type $\left(p^{s}, p\right)$. Since $H^{*} \cap U=\langle z\rangle \leq \mathrm{Z}(G)$, it follows that $H^{*} U$ is the unique normal subgroup of $G$ which contains $H^{*}$ with $\left|\left(H^{*} U\right): H^{*}\right|=p$. By our assumptions, we have that $\Omega_{1}\left(H^{*}\right)=\langle z, i\rangle$ centralizes $U$. Thus $\mathrm{C}_{G}(U) \geq L\langle i\rangle$ and since $u^{\prime}$ commutes with $i$ and $t$, we get together with $[i, t]=u^{\prime}$ that $D=\langle i, t\rangle \cong \mathrm{D}_{8}$ if $p=2$ and $D=\langle i, t\rangle \cong \mathrm{S}\left(p^{3}\right)$ if $p>2$ and in any case we get $D^{\prime}=\mathrm{Z}(D)=\left\langle u^{\prime}\right\rangle$.

If $D \cong \mathrm{D}_{8}$, then our assumptions imply $D \unlhd G$ and if $D \cong \mathrm{S}\left(p^{3}\right)$, then Proposition 1 gives that $D \unlhd G$. Hence in any case we have $D \unlhd G$ and so $D^{\prime}=\left\langle u^{\prime}\right\rangle \leq \mathrm{Z}(G)$. This gives that $G^{\prime}=U=\langle z\rangle \times\left\langle u^{\prime}\right\rangle \leq \mathrm{Z}(G)$ and therefore $G$ is of class 2 with $\mho_{1}(G) \leq \mathrm{Z}(G)$. Since $D \cap G^{\prime}=\left\langle u^{\prime}\right\rangle$, it follows that no element in $G$ induces an outer automorphism on $D$. We get $G=D * C$, where $C=\mathrm{C}_{G}(D)$ and $C \cap D=\left\langle u^{\prime}\right\rangle$.

Note that $\langle h\rangle U \leq C$ and $\mathrm{C}_{G}(t)=C \times\langle t\rangle$, which together with the fact that no element in $G-K$ centralizes $t$ implies that $\mathrm{C}_{G}(t)=K$. Also, we have $\left|G: \mathrm{C}_{G}(i)\right|=p$ and so if $K$ would be abelian, then $C=\mathrm{C}_{K}(i)$ is abelian and then $G^{\prime}=D^{\prime}=\left\langle u^{\prime}\right\rangle$ is of order $p$, a contradiction. Hence $K$ is nonabelian and so $K^{\prime}=\langle z\rangle=C^{\prime}$ since $K=C \times\langle t\rangle$. If $\langle h\rangle \leq \mathrm{Z}(K)$, then $L \leq \mathrm{Z}(K)$ and so the fact that $K / L$ is cyclic gives that $K$ is abelian, a contradiction. Hence we get $\langle h\rangle \not \subset \mathrm{Z}(K)$ and so, in particular, we have $K>L$.

We have $K=\mathrm{C}_{K}(i) \times\langle t\rangle$ and since $K / H$ is cyclic of order $\geq p^{2}$ and

$$
K / H \cong \mathrm{C}_{K}(i) / \mathrm{C}_{H}(i)=\mathrm{C}_{K}(i) /\langle h\rangle,
$$

we may choose $k \in \mathrm{C}_{K}(i)=C$ so that $\langle k\rangle$ covers $\mathrm{C}_{K}(i) /\langle h\rangle$ and $[h, k]=z$. Since

$$
C=\mathrm{C}_{K}(i)=\langle h, k\rangle \text { with }[h, k]=z,\langle z\rangle=\langle h\rangle \cap U \text { and } U=\Omega_{1}(C) \leq \mathrm{Z}(G)
$$

and noting that $\Omega_{1}(K)=U \times\langle t\rangle \cong \mathrm{E}_{p^{3}}$, it follows that $C$ is metacyclic minimal nonabelian without a cyclic subgroup of index $p$. Hence we may set

$$
C=\left\langle a, b \mid a^{p^{\alpha}}=b^{p^{\beta}}=1,[a, b]=z=a^{p^{\alpha-1}}\right\rangle,
$$

where $\alpha \geq 2, \beta \geq 2$ and $b^{p^{\beta-1}}=u \in U-\langle z\rangle$. Also we know that we have $G=C *\langle i, t\rangle$ with $C \cap\langle i, t\rangle=\left\langle u^{\prime}\right\rangle, u^{\prime} \in U-\langle z\rangle$ and $D=\langle i, t\rangle \cong \mathrm{D}_{8}$ or $\mathrm{S}\left(p^{3}\right)$. 
We consider the subgroup $H_{1}=\langle b\rangle \times\langle i\rangle \cong \mathrm{C}_{p^{\beta}} \times \mathrm{C}_{p}, \beta \geq 2$. Since $H_{1} \cap C=\langle b\rangle$ and $[a, b]=z \notin H_{1}$, it follows that $H_{1}$ is non-normal in $G$. Suppose that $H_{1}$ is not a maximal non-normal subgroup in $G$. Then there is an element $b^{\prime} \in G$ such that $b=i^{\gamma}\left(b^{\prime}\right)^{p}$, where $\gamma$ is an integer mod $p$ and $\left(b^{\prime}\right)^{p} \in \mho_{1}(G) \leq \mathrm{Z}(G)$. Then we get

$$
[a, b]=\left[a, i^{\gamma}\left(b^{\prime}\right)^{p}\right]=[a, i]^{\gamma}=1
$$

a contradiction. Hence $H_{1}$ is a maximal non-normal subgroup in $G$. By Proposition $6, H_{1}$ possesses a $G$-invariant subgroup $\left\langle b i^{\delta}\right\rangle$ of index $p$, where $\delta$ is an integer $\bmod p$ and $\Omega_{1}\left(\left\langle b i^{\delta}\right\rangle\right)=\langle u\rangle$. On the other hand, we have $\left[a, b i^{\delta}\right]=$ $[a, b]=z$, a contradiction. We have proved that there are no elements of order $p$ in $G-K$.

Now assume that $G$ is of class 3 . In that case no element in $U-\langle z\rangle$ is a $p$ th power of an element in $G$. Indeed, if there is $x \in G$ such that $x^{p} \in U-\langle z\rangle$, then we consider the subgroup $U\langle x\rangle \unlhd G$ of order $p^{3}$. Since $\langle z\rangle \leq \mathrm{Z}(G)$ and $x$ commutes with $x^{p}$, it follows that $U\langle x\rangle$ is abelian of type $\left(p^{2}, p\right)$. But then we get $\mho_{1}(U\langle x\rangle)=\left\langle x^{p}\right\rangle$ is normal in $G$ and so $G^{\prime}=U \leq \mathrm{Z}(G)$, a contradiction.

Note that $G / K \cong \mathrm{C}_{p}$ acts transitively on $p$ subgroups of order $p^{2}$ in $S=U \times\langle t\rangle$ which contain $\langle z\rangle$ and which are distinct from $U$. Assume for a moment that $t \notin \mathrm{Z}(K)$. Then we have $K^{\prime}=\langle z\rangle$ and $K>L$. Let $k \in K-\mathrm{C}_{K}(t)$ so that $\langle k\rangle$ covers $K / H$. Suppose that $\left\langle k^{\prime}\right\rangle=\Omega_{1}(\langle k\rangle) \not \leq U$. Then we have $k^{\prime} \in \mathrm{Z}(K)$ and if $U \not \subset \mathrm{Z}(K)$, then $\Omega_{1}(\mathrm{Z}(K))=\left\langle z, k^{\prime}\right\rangle \unlhd G$, a contradiction. Hence $U \leq \mathrm{Z}(K)$ and so $S \leq \mathrm{Z}(K)$ which implies that $t \in \mathrm{Z}(K)$, a contradiction. Thus we have $\Omega_{1}(\langle k\rangle) \leq U$ and so $\Omega_{1}(\langle k\rangle)=\langle z\rangle$ and $o(k) \geq p^{3}$. Since $\langle[k, t]\rangle=\langle z\rangle$, we have

$$
\langle k, t\rangle \cong \mathrm{M}_{p^{m}}, m \geq 4 .
$$

On the other hand, for an element $g \in G-K$ we have $[g, t]=u^{\prime} \in U-\langle z\rangle$ and so $\langle k, t\rangle$ is not normal in $G$, contrary to our assumptions. We have proved that $t \in \mathrm{Z}(K)$ and so we have $\mathrm{C}_{G}(t)=K$.

If $U \not Z \mathrm{Z}(K)$, then $H_{0}=\Omega_{1}(\mathrm{Z}(K)) \unlhd G$, a contradiction. Hence we have $U \leq \mathrm{Z}(K)$ and so $S=\Omega_{1}(\mathrm{Z}(K))=\Omega_{1}(G)$. Let $x \in G-K$ so that we have $\mathrm{C}_{U}(x)=\langle z\rangle$ and therefore, by the above, $\mathrm{C}_{S}(x)=\langle z\rangle$. In particular, we get $p>2$ and $\Omega_{1}(\langle x\rangle)=\langle z\rangle$.

Suppose that for some $y \in K$ we have $y^{p} \in S-U$. Then we have $\langle y\rangle S \unlhd G$ and

$$
\mho_{1}(\langle y\rangle S)=\left\langle y^{p}\right\rangle \leq \mathrm{Z}(G)
$$

a contradiction. Hence for each element $x \in G$ of composite order, the socle $\Omega_{1}(\langle x\rangle)$ is equal $\langle z\rangle$.

Assume that $\langle h\rangle \not Z \mathrm{Z}(K)$ so that we have $K>L$. Let $k \in K$ be such that $\langle k\rangle$ covers $K / H$ and since $\Omega_{1}(\langle k\rangle)=\langle z\rangle$, we get $o(k) \geq p^{3}$. It follows that $\langle h, k\rangle$ is a splitting metacyclic minimal nonabelian subgroup with $\langle[h, k]\rangle=$ 
$\langle z\rangle$. We may set

$$
\langle h, k\rangle=\left\langle a, b \mid a^{p^{\alpha}}=b^{p^{\beta}}=1,[a, b]=z=a^{p^{\alpha-1}}\right\rangle,
$$

where $\alpha \geq 3$ and $\beta \geq 1$. By the previous paragraph, we must have $\beta=1$ and then $b \in \mathrm{Z}(K)$, a contradiction.

We have proved that $h \in \mathrm{Z}(K)$ and so $L \leq \mathrm{Z}(K)$ which together with the fact that $K / L$ is cyclic implies that $K$ is abelian. Hence $K$ is abelian of rank 3 and therefore we may set

$$
K=\langle a\rangle \times\langle u\rangle \times\langle t\rangle \text { with } \Omega_{1}(\langle a\rangle)=\langle z\rangle, o(a) \geq p^{s}, \text { and }\langle z, u\rangle=U .
$$

Since $[t, g] \in U-\langle z\rangle$ for each element $g \in G-K$, we have that $\langle a\rangle \times\langle t\rangle$ is non-normal in $G$ which together with the maximality of $|H|$ gives $o(a)=p^{s}$ and so we have $K=L$.

Let $g \in G-K$. Since $\mathrm{C}_{S}(g)=\langle z\rangle$, it follows that $\mathrm{C}_{K}(g)$ is cyclic. By Lemma 1.1 in [1], $\mathrm{C}_{K}(g)=\left\langle h^{\prime}\right\rangle$ covers $K / S$ and so $\left\langle h^{\prime}\right\rangle \cong \mathrm{C}_{p^{s}}$ and $\left\langle h^{\prime}\right\rangle=\mathrm{Z}(G)$ so that $g^{p} \in\left\langle h^{\prime}\right\rangle$. But there are no elements of order $p$ in $G-K$ and so $\left\langle g, h^{\prime}\right\rangle=\langle g\rangle$ is cyclic of order $p^{s+1}$. We may assume without loss of generality that $g^{p}=h$. Then we may set $[g, t]=u \in U-\langle z\rangle$ and $[u, g]=z$, where $\langle z\rangle=\Omega_{1}(\langle g\rangle)$. The group $G$ has a maximal subgroup

$$
\mathrm{M}_{p^{s+2}} \cong\left\langle g, u \mid g^{p^{s+1}}=u^{p}=1,[u, g]=z,\langle z\rangle=\Omega_{1}(\langle g\rangle)\right\rangle,
$$

where $p>2, s \geq 2$ and $G=\langle g, u\rangle\langle t\rangle$ with $o(t)=p,[g, t]=u$ and $[u, t]=1$. We have obtained the groups stated in part (a) of our proposition. It turns out that these groups are actually $\mathrm{A}_{2}$-groups which are defined in Proposition 71.3(i) in [2]. Conversely, it is easy to check that these groups satisfy our condition $(*)$.

From now on we may assume that $G$ is of class 2 . Since $G^{\prime}=U \cong \mathrm{E}_{p^{2}}$, we also have $\mho_{1}(G) \leq \mathrm{Z}(G)$. Also we have $\Omega_{1}(\mathrm{Z}(G))=U$ and so no element in $S-U$ is a $p$-th power of any element in $G$.

(i) Assume that $K=L$. In this case Lemma 1.1 in [1] gives that $|G / \mathrm{Z}(G)|=p^{3}$. We have $\langle h\rangle \unlhd G$ but $\langle h\rangle \not \mathbf{Z}(G)$ and so we have $\mathrm{Z}(G)=U\left\langle h^{p}\right\rangle$. Hence for each $g \in G-K$, we get $1 \neq g^{p} \in U\left\langle h^{p}\right\rangle$.

(i1) First suppose that $1 \neq g^{p} \in\left\langle h^{p}\right\rangle \geq\langle z\rangle$. Since there are no elements of order $p$ in $\langle g, h\rangle-\langle h\rangle$ and $\langle g, h\rangle$ is nonabelian (because $\langle h\rangle \not \mathbf{Z}(G)$ ) with $\Omega_{1}(\langle g, h\rangle)=\langle z\rangle$, it follows that we have $p=2$ and $\langle g, h\rangle \cong \mathrm{Q}_{8}$. Hence $\langle h\rangle \cong \mathrm{C}_{4}, g^{2}=z,[g, h]=z$ and $[g, t]=u \in U-\langle z\rangle$. We have obtained the special group of order $2^{5}$ stated in part (b) of our proposition and this group satisfies our condition $(*)$.

(i1) Now we assume that $g^{p} \in\left(U\left\langle h^{p}\right\rangle\right)-\left\langle h^{p}\right\rangle$ so that we may set $g^{p}=u h^{\prime}$, where $u \in U-\langle z\rangle,\langle z\rangle=\Omega_{1}(\langle h\rangle)$ and $h^{\prime} \in\left\langle h^{p}\right\rangle$. Let $h_{0}$ be an element in $\langle h\rangle$ such that $h_{0}^{p}=\left(h^{\prime}\right)^{-1}$. Then we replace $g$ with $g h_{0} \in G-K$ and we compute

$$
\left(g h_{0}\right)^{p}=g^{p} h_{0}^{p}\left[h_{0}, g\right]^{\left(\begin{array}{c}
p \\
2
\end{array}\right)}=\left(u h^{\prime}\right)\left(h^{\prime}\right)^{-1} z^{\prime}=u z^{\prime} \in U-\langle z\rangle,
$$


where

$$
\left[h_{0}, g\right]^{\left(\begin{array}{c}
p \\
2
\end{array}\right)}=z^{\prime} \in\langle z\rangle .
$$

It follows that in this case we may choose from the start an element $g \in G-K$ so that $g^{p}=u \in U-\langle z\rangle$. Then we have $[g, t]=u z^{i}$ for some integer $i \bmod$ $p$ ( where we have replaced $t$ with a suitable power $t^{j}(j \not \equiv 0 \bmod p)$. Let $h^{*} \in\langle h\rangle$ be such that $\left(h^{*}\right)^{p}=z^{i}$.

Assume that either $p>2$ or $p=2$ and $s \geq 3$ (where in the last case we have $\left.\left[h^{*}, g\right]=1\right)$. Then we consider the subgroup $\left\langle g^{\prime}, t\right\rangle$, where $g^{\prime}=g h^{*} \in$ $G-K$. We have

$$
\left(g^{\prime}\right)^{p}=g^{p}\left(h^{*}\right)^{p}\left[h^{*}, g\right]^{\left(\begin{array}{l}
p \\
2
\end{array}\right)}=u z^{i}=[g, t]=\left[g h^{*}, t\right]=\left[g^{\prime}, t\right],
$$

and so we get $\left\langle g^{\prime}, t\right\rangle \cong \mathrm{D}_{8}$ if $p=2$ and $\left\langle g^{\prime}, t\right\rangle \cong \mathrm{M}_{p^{3}}$ if $p>2$. On the other hand, $1 \neq\left[h, g^{\prime}\right] \in\langle z\rangle$ and so $\left\langle g^{\prime}, t\right\rangle$ is non-normal in $G$, contrary to our assumptions.

We have proved that we must have $p=2$ and $s=2$ so that we have $\langle h\rangle \cong \mathrm{C}_{4}$ and $G$ is a special group of order $2^{5}$ with $g^{2}=u \in U-\langle z\rangle, h^{2}=z$, $[g, h]=z$ and $[g, t]=u z^{i}, i=0,1$. However, if $i=0$, then $\langle g, t\rangle \cong \mathrm{D}_{8}$ is non-normal in $G$, a contradiction. Thus we have $i=1$ and so $[g, t]=u z$. The structure of $G$ is uniquely determined.

We claim that the special 2-group obtained in the previous paragraph is in fact isomorphic to the special group of order $2^{5}$ from part (i1) of our proof. Indeed, set $g^{\prime}=g t$ and $u^{\prime}=u z$. Then we have

$$
\begin{aligned}
& \left(g^{\prime}\right)^{2}=(g t)^{2}=u(u z)=z=h^{2}, \\
& {\left[g^{\prime}, h\right]=[g t, h]=z,} \\
& {\left[g^{\prime}, t\right]=[g t, t]=u z=u^{\prime} .}
\end{aligned}
$$

In addition we have $\left[g^{\prime}, u^{\prime}\right]=[h, t]=1$ and so writing again $g, u$ instead of $g^{\prime}, u^{\prime}$, respectively, we see that we have obtained the relations for the special group of order $2^{5}$ defined in $(i 1)$.

From now on we shall always assume that $K>L$.

(ii) Suppose that $G / L$ is cyclic of order $\geq p^{2}$. Let $g \in G-K$ so that $\langle g\rangle$ covers $G / L$. But $g^{p} \in \mathrm{Z}(G)$ and $\left\langle g^{p}\right\rangle$ covers $K / L \neq\{1\}$. Since $K / H$ is cyclic of order $\geq p^{2}$, it follows that $\left\langle g^{p}\right\rangle$ covers $K / H$ and so $K=H\left\langle g^{p}\right\rangle$ is abelian. Since $G^{\prime}=U \cong \mathrm{E}_{p^{2}}$, Lemma 1.1 in [1] implies that $|G: \mathrm{Z}(G)|=p^{3}$. On the other hand, $\left\langle h^{p}, g^{p}\right\rangle \leq \mathrm{Z}(G)$ and $\left|K_{1}:\left\langle h^{p}, g^{p}\right\rangle\right|=p$, where $K_{1}=\left\langle h, g^{p}\right\rangle$ and $K=\langle t\rangle \times K_{1}$ is of rank 3 . It follows that $\mathrm{Z}(G)=\left\langle h^{p}, g^{p}\right\rangle$. In particular, (since $U \leq \mathrm{Z}(G)$ ) we must have $U \leq\left\langle h^{p}, g^{p}\right\rangle$ so that $\Omega_{1}\left(K_{1}\right)=U$ and $h \notin \mathrm{Z}(G)$. We may set $[g, h]=z$. There are exactly $p$ conjugate classes of non-central subgroups of order $p$ in $G$ with the representatives $\left\langle t z^{i}\right\rangle, 0 \leq i \leq p-1$. It follows (using also Proposition 6) that any abelian maximal non-normal subgroup in $G$ of type $\left(p^{r}, p\right), r \geq 2$ is contained in $\mathrm{C}_{G}\left(t z^{i}\right)=K$. 
Suppose that $K_{1}$ is of exponent $p^{r}$, where $r>s$. Let $k$ be an element of order $p^{r}$ in $K_{1}$ and consider the subgroup $\langle t\rangle \times\langle k\rangle$. If $\langle t\rangle \times\langle k\rangle$ is non-normal in $G$, then $\langle t\rangle \times\langle k\rangle$ is maximal non-normal in $G$ of order $\rangle|H|=p^{s}$, contrary to our assumptions. Hence we have $\langle t\rangle \times\langle k\rangle \unlhd G$. Since $[g, t] \in U-\langle z\rangle$, it follows that $\Omega_{1}(\langle k\rangle)=\langle u\rangle$ with $u \in U-\langle z\rangle$. Since $[g, h]=z$, we have $\left[g, K_{1}\right]=\langle z\rangle$ and so the fact that $k \in K_{1}$ implies that $[g, k] \in\langle z\rangle$. But we have $\Omega_{1}(\langle t, k\rangle)=\langle t, u\rangle$ and so $[g, k]=1$ and therefore $k \in \mathrm{Z}(G)$. Now consider the subgroup $\langle t\rangle \times\langle h k\rangle$, where $h k \in K_{1} o(h k)=p^{r}$ and $\Omega_{1}(\langle h k\rangle)=\langle u\rangle$. If $\langle t\rangle \times\langle h k\rangle$ is not normal in $G$, then $\langle t\rangle \times\langle h k\rangle$ is maximal non-normal in $G$ of order $>|H|$, a contradiction. Hence we have $\langle t\rangle \times\langle h k\rangle \unlhd G$. But $[g, h k]=[g, h][g, k]=z$ and $z \notin \Omega_{1}(\langle t\rangle \times\langle h k\rangle)=\langle t, u\rangle$, a contradiction. We have proved that $\exp (K)=\exp \left(K_{1}\right)=p^{s}$ and therefore $o(g) \leq p^{s+1}$ and all elements in $G-K$ are of order $\leq p^{s+1}$.

There are elements of order $p^{s}$ or $p^{s+1}$ in $G-K$. Indeed, assume that $o(g) \leq p^{s-1}$ for some $g \in G-K$. In that case we must have $s \geq 3$ since $\Omega_{1}(G)=U \times\langle t\rangle$. Then we compute

$$
(g h)^{p^{s-1}}=g^{p^{s-1}} h^{p^{s-1}}[h, g]^{\left(\begin{array}{c}
p^{s-1} \\
2
\end{array}\right)}=h^{p^{s-1}}=z,
$$

where $\langle z\rangle=\Omega_{1}(\langle h\rangle)$ and so we get $o(g h)=p^{s}$.

If there is an element $g \in G-K$ of order $p^{s+1}$, then all elements in $G-K$ are of order $p^{s+1}$. Indeed, for any $x \in K$ and and any integer $i \not \equiv 0(\bmod$ $p)$ we have:

$$
\left(g^{i} x\right)^{p^{s}}=\left(g^{i}\right)^{p^{s}} x^{p^{s}}\left[x, g^{i}\right]^{\left(\begin{array}{c}
p^{s} \\
2
\end{array}\right)}=\left(g^{i}\right)^{p^{s}} \neq 1 .
$$

(ii1) Suppose that $G-K$ contains elements of order $p^{s}$. Let $g$ be an element of the minimal possible order $p^{r}$ in $G-K$. Then we have $3 \leq r \leq s$. Indeed, $\langle g\rangle$ covers $G / L$ (which is cyclic of order $\geq p^{2}$ ) and there are no elements of order $p$ in $G-L$ and so $o(g) \geq p^{3}$.

The element $g^{p^{r-1}}$ is of order $p$ and is contained in $U$. Assume that $g^{p^{r-1}}=z$, where $\langle z\rangle=\Omega_{1}(\langle h\rangle)$. Let $h^{\prime}$ be an element in $\langle h\rangle$ such that $\left(h^{\prime}\right)^{p^{r-1}}=z^{-1}$. Then we compute (noting that $r \geq 3$ ):

$$
\left(h^{\prime} g\right)^{p^{r-1}}=\left(h^{\prime}\right)^{p^{r-1}} g^{p^{r-1}}\left[g, h^{\prime}\right]^{\left(\begin{array}{c}
p^{r-1} \\
2
\end{array}\right)}=z^{-1} z=1,
$$

and so $o\left(h^{\prime} g\right) \leq p^{r-1}$, a contradiction. We have proved that $\langle g\rangle$ splits over $\langle h\rangle$ and so we have $\Omega_{1}(\langle g\rangle)=\langle u\rangle$ with $u \in U-\langle z\rangle$.

Set $h^{p^{s-1}}=z, s \geq 3$, and then replacing $g$ with $g^{j}$ for some integer $j \not \equiv 0(\bmod p)$, we see that we may set $[g, h]=z$. Replacing $t$ with $t^{l}$ for some suitable integer $l \not \equiv 0(\bmod p)$, we may assume that $[g, t]=u z^{i}$ for some integer $i(\bmod p)$. If $[g, t]=u($ i.e. $i \equiv 0(\bmod p))$, then we have $\langle g, t\rangle \cong \mathrm{M}_{p^{r+1}}, r \geq 3$. But $[g, h]=z \notin\langle g, t\rangle$ and so $\langle g, t\rangle$ is not normal in $G$, contrary to our assumptions. Hence we have $i \not \equiv 0(\bmod p)$.

Assume that $r=s$ and so $o(g)=p^{s}$. We set $g^{p^{s-1}}=u$ and then changing $t$ with a suitable power $t^{j}, j \not \equiv 0(\bmod p)$, we may set $[g, t]=u z^{i}$ with $i \not \equiv 0$ 
$(\bmod p)$. Let $h^{\prime} \in\langle h\rangle$ be such that $\left(h^{\prime}\right)^{p^{s-1}}=z^{i}$. Then we have (noting that $s \geq 3)$ :

$$
\left(g h^{\prime}\right)^{p^{s-1}}=u z^{i}\left[h^{\prime}, g\right]^{\left(p^{s-1}\right)}=u z^{i}
$$

and since $\left[g h^{\prime}, t\right]=[g, t]=u z^{i}$, we obtain that $\left\langle g h^{\prime}, t\right\rangle \cong \mathrm{M}_{p^{s+1}}$. On the other hand, we have $1 \neq\left[g h^{\prime}, h\right] \in\langle z\rangle$ and so $\left\langle g h^{\prime}, t\right\rangle$ is non-normal in $G$, a contradiction. We have proved that we must have $o(g)=p^{r}$ with $3 \leq r<s$ and this gives $s \geq 4$. We have obtained the groups stated in part (c) of our proposition which obviously satisfy our condition $(*)$.

(ii2) Suppose that all elements in $G-K$ are of order $p^{s+1}$.

(ii2a) First assume that there is $g \in G-K$ such that $\langle g\rangle$ splits over $\langle h\rangle$. We may choose a generator $g$ in $\langle g\rangle$ so that $[g, h]=z=h^{p^{s-1}}, s \geq 2$. Then we set $u=g^{p^{s}} \in U-\langle z\rangle$ and we may choose a generator $t \in\langle z\rangle$ so that $[g, t]=u z^{i}$, where $i$ is an integer $\bmod p$. Suppose that $i \equiv 0(\bmod p)$. Then we have $\langle g, t\rangle \cong \mathrm{M}_{p^{s+2}}$. But $[g, h]=z \notin\langle g, t\rangle$ and so $\langle g, t\rangle$ is not normal in $G$, contrary to our assumptions. Hence we have $i \not \equiv 0(\bmod p)$. Note that the socle $\Omega_{1}(\langle x\rangle)$ is equal $\langle u\rangle$ for each $x \in G-K$.

Consider the subgroup $X=\left\langle t, h^{\alpha} g^{p}\right\rangle \cong \mathrm{C}_{p} \times \mathrm{C}_{p^{s}}$, where $g^{p} \in \mathrm{Z}(G)$ and $\alpha$ is any fixed integer with $\alpha \not \equiv 0(\bmod p)$. We have for every integer $j(\bmod$ $p)$ :

$$
\left(t^{j} h^{\alpha} g^{p}\right)^{p^{s-1}}=\left(h^{p^{s-1}}\right)^{\alpha} g^{p^{s}}=z^{\alpha} u
$$

and so $\left\langle t^{j} h^{\alpha} g^{p}\right\rangle \cong \mathrm{C}_{p^{s}}$ is a maximal cyclic subgroup in $G$ since its socle is $\left\langle z^{\alpha} u\right\rangle$. We have $\Omega_{1}(X)=\left\langle t, z^{\alpha} u\right\rangle$ and

$$
\left[g, h^{\alpha} g^{p}\right]=\left[g, h^{\alpha}\right]=z^{\alpha} \notin X
$$

implies that $X$ is not normal in $G$. This gives

$$
\mathrm{N}_{G}(X)=\mathrm{N}_{G}\left(\Omega_{1}(X)\right)=K .
$$

We have $[g, t]=u z^{i}$ and so $z^{i} u \notin \Omega_{1}(X)=\left\langle t, z^{\alpha} u\right\rangle$. In particular, $i \not \equiv \alpha(\bmod$ $p)$ for any integer $\alpha \neq \equiv 0(\bmod p)$. But this implies that we must have $i \equiv 0$ $(\bmod p)$, a contradiction.

(ii2b) We have proved that for each $g \in G-K,\langle g\rangle$ does not split over $\langle h\rangle$. Hence we have:

$$
\langle g\rangle \cap\langle h\rangle \geq\langle z\rangle,\langle g, h\rangle^{\prime}=\langle z\rangle=\Omega_{1}(\langle h\rangle)
$$

and therefore

$$
\langle g\rangle \unlhd\langle g, h\rangle \text { with } p \leq|\langle g, h\rangle:\langle g\rangle| \leq p^{s-1} .
$$

Since $\left\langle g^{p}\right\rangle$ is of order $p^{s}=\exp \left(\left\langle g^{p}, h\right\rangle\right)$, it follows that $\left\langle g^{p}\right\rangle$ splits in $\left\langle g^{p}, h\right\rangle$ and so we have:

$$
\begin{gathered}
\left\langle g^{p}, h\right\rangle=\langle k\rangle \times\left\langle g^{p}\right\rangle \text { with } K=\langle t\rangle \times\left(\langle k\rangle \times\left\langle g^{p}\right\rangle\right) \text { and } \\
\langle k\rangle\langle g\rangle=\langle g, h\rangle \text { with }\langle k\rangle \cap\langle g\rangle=\{1\} .
\end{gathered}
$$


Because $\langle[k, g]\rangle=\langle z\rangle$ and $\Omega_{1}(\langle k\rangle\langle g\rangle)=U \leq \mathrm{Z}(G)$, we get $o(k)=p^{r}, 2 \leq r \leq$ $s-1$ and so $s \geq 3$. We may set $u=k^{p^{r-1}} \in U-\langle z\rangle$ and $[g, k]=z=g^{p^{s}}$. Also note that the socle $\Omega_{1}(\langle x\rangle)$ for each $x \in G-K$ is equal $\langle z\rangle$.

We may choose a suitable generator $t$ in $\langle t\rangle$ so that $[g, t]=u z^{i}$ for some integer $i \bmod p$. Consider the subgroup $Y=\langle k\rangle \times\langle t\rangle \cong \mathrm{C}_{p} \times \mathrm{C}_{p^{r}}, 2 \leq r \leq s-1$, which is not normal in $G$ since $[g, k]=z \notin Y$. We have $\mathrm{N}_{G}(Y)=K$ and so $\mathrm{N}_{G}(\langle t, u\rangle)=K$, where $\langle t, u\rangle=\Omega_{1}(Y)$. We have $[g, t]=u z^{i} \notin \Omega_{1}(Y)$ and so we must have $i \not \equiv 0(\bmod p)$.

Choose an element $g^{\prime}$ in $\left\langle g^{p}\right\rangle$ such that $o\left(g^{\prime}\right)=p^{r}$ and $\left(g^{\prime}\right)^{p^{r-1}}=z$ and note that $g^{\prime} \in \mathrm{Z}(G)$. Now we consider for each $\alpha \neq 0(\bmod p)$ the subgroup $V=\left\langle k^{\alpha} g^{\prime}\right\rangle \times\langle t\rangle \cong \mathrm{C}_{p^{r}} \times \mathrm{C}_{p}$ with $\left(k^{\alpha} g^{\prime}\right)^{p^{r-1}}=u^{\alpha} z$ so that $\Omega_{1}(V)=\left\langle t, u^{\alpha} z\right\rangle$. Since $\left[g, k^{\alpha} g^{\prime}\right]=z^{\alpha} \notin \Omega_{1}(V)$, we have $\mathrm{N}_{G}(V)=K$ and so also $\mathrm{N}_{G}\left(\left\langle t, u^{\alpha} z\right\rangle\right)=$ $K$. Because $[g, t]=u z^{i}$, it follows that $u z^{i} \notin\left\langle u^{\alpha} z\right\rangle$ for each $\alpha \neq \equiv 0(\bmod p)$. We can find an integer $j \not \equiv 0(\bmod p)$ so that $i j \equiv 1(\bmod p)$. We get

$$
\left(u z^{i}\right)^{j}=u^{j} z^{i j}=u^{j} z \notin\left\langle u^{\alpha} z\right\rangle
$$

for each $\alpha \not \equiv 0(\bmod p)$, a contradiction.

(iii) We consider the remaining case, where $G / L$ is not cyclic and $G>L$. Since $G / L$ is abelian and $K / L \neq\{1\}$ is cyclic, it follows that $G / L$ splits over $K / L$ and so we have $G=K G_{0}$ with $K \cap G_{0}=L$ and $\left|G_{0}: L\right|=p$. Also, $K / H$ is cyclic of order $\geq p^{2}$ and we have:

$$
\begin{gathered}
H=\langle h\rangle \times\langle t\rangle \cong \mathrm{C}_{p^{s}} \times \mathrm{C}_{p}, s \geq 2, \text { where } \mathrm{C}_{p^{s}} \cong\langle h\rangle \unlhd G,\langle t\rangle \cong \mathrm{C}_{p}, \\
\Omega_{1}(H)=\langle z\rangle, G^{\prime}=U \cong \mathrm{E}_{p^{2}}, L=U H \text { is abelian and } U \leq \mathrm{Z}(G) .
\end{gathered}
$$

(iii) Suppose first that $\langle h\rangle \not \subset \mathrm{Z}\left(G_{0}\right)$ so that we have $U=G_{0}^{\prime} \cong \mathrm{E}_{p^{2}}$ and therefore by (i) we get $p=2$ and $G_{0}$ is the uniquely determined special 2 -group of order $2^{5}$ (stated in part (b) of our proposition):

$$
\begin{gathered}
L=\langle h\rangle \times\langle u\rangle \times\langle t\rangle \cong \mathrm{C}_{4} \times \mathrm{C}_{2} \times \mathrm{C}_{2},\langle h\rangle \cong \mathrm{C}_{4}, h^{2}=z,\langle u\rangle \cong\langle t\rangle \cong \mathrm{C}_{2}, \\
G_{0} \cong L\langle g\rangle \text { with } g^{2}=z,[g, h]=z,[g, u]=1, \text { and }[g, t]=u .
\end{gathered}
$$

Since $\mathrm{Z}\left(G_{0}\right)=U$, it follows that for each $x \in K-L$ such $x^{2} \in L$, we must have $1 \neq x^{2} \in U$. Let $k \in K-L$ be such that $\langle k\rangle$ covers the cyclic group $K / H$ of order $\geq 4$. Thus $\Omega_{1}(\langle k\rangle)=\langle u\rangle$ or $\langle u z\rangle$ and so $K$ splits over $H$.

Because $\mathrm{C}_{G_{0}}(g)=U\langle g\rangle$ and so $\left|G_{0}: \mathrm{C}_{G_{0}}(g)\right|=4$, we get together with $\left|G^{\prime}\right|=4$ that $\left|G: \mathrm{C}_{G}(g)\right|=4$. But we have $G=K\langle g\rangle$ and so $\mathrm{C}_{G}(g)=$ $\mathrm{C}_{K}(g)\langle g\rangle$ which implies that $\left|K: \mathrm{C}_{K}(g)\right|=4$. On the other hand, we have $\left|H: \mathrm{C}_{H}(g)\right|=4$ and therefore $\mathrm{C}_{K}(g)$ covers $K / H$. It follows that we may choose our element $k \in \mathrm{C}_{K}(g)$ such that $\langle k\rangle$ covers $K / H$. Hence we may assume $[g, k]=1$.

Case (1). Suppose that $|K: L|>2$ so that $o(k) \geq 8$. Then there is an element $k^{\prime}$ of order 4 in $\langle k\rangle$ such that $k^{\prime} \in \mathrm{Z}(G)$. Note that $(t g)^{2}=u z$ and so 
if $\left(k^{\prime}\right)^{2}=u z$, then $k^{\prime}(t g)$ is an involution in $G-K$, a contradiction. Hence we must have in this case $\left(k^{\prime}\right)^{2}=u$. We set $o(k)=2^{n}, n \geq 3$, and then we have $k^{2^{n-1}}=u$. Assume for a moment that that $[k, h]=[k, t]=1$ which together with $[k, g]=1$ (from the previous paragraph) then implies that $k \in \mathrm{Z}(G)$. In that case we have $(g k)^{2^{n-1}}=u$ and $[g k, t]=u$ so that $\langle g k, t\rangle \cong \mathrm{M}_{2^{n+1}}$ with $n \geq 3$. But $[h, g k]=z \notin\langle g k, t\rangle$ and so $\langle g k, t\rangle$ is not normal in $G$, contrary to our assumptions. We have proved that $k \notin \mathrm{Z}(G)$.

Assume that $[k, t]=1$. Then we have $[k, h]=z$. Consider in this case the subgroup

$$
\langle t\rangle \times\langle k\rangle, \text { where } o(k)=2^{n}=\exp (\mathrm{G}), n \geq 3 .
$$

Since $[h, k]=z \notin\langle t, k\rangle$, it follows that $\langle t, k\rangle$ is a maximal non-normal subgroup in $G$ of order $>|H|$, contrary to our assumptions. We have proved that we must have $[k, t]=z$ (noting that we have $K^{\prime} \leq\langle z\rangle$ ).

Now we consider the subgroup $\langle t\rangle \times\left\langle h k^{\prime}\right\rangle$, where $k^{\prime}$ is an element of order 4 in $\langle k\rangle$ and $k^{\prime} \in \mathrm{Z}(G)$. Here we have $\Omega_{1}\left(\left\langle t, h k^{\prime}\right\rangle\right)=\langle t, u z\rangle$. Because $[g, t]=u$, it follows that $\left\langle t, h k^{\prime}\right\rangle \cong \mathrm{C}_{2} \times \mathrm{C}_{4}$ is abelian non-normal in $G$. By the maximality of $|H|$, it follows that $\left\langle t, h k^{\prime}\right\rangle$ is a maximal non-normal subgroup in $G$. Then Proposition 6 implies that either $\left\langle h k^{\prime}\right\rangle \unlhd G$ or $\left\langle t h k^{\prime}\right\rangle \unlhd G$. But $\left[h k^{\prime}, g\right]=z$ and so $\left\langle h k^{\prime}\right\rangle$ is not normal in $G$. Hence we must have $\left\langle t h k^{\prime}\right\rangle \unlhd G$. From $\left[t h k^{\prime}, k\right]=z[h, k]$ follows that $[h, k]=z$.

Finally assume that $n>3$ so that the subgroup $\langle t\rangle \times\left\langle k^{2}\right\rangle \cong \mathrm{C}_{2} \times \mathrm{C}_{2^{n-1}}$ is non-normal in $G$ ( since $[t, k]=z)$, contrary to the maximality of $|H|$. Hence we get $n=3, o(k)=8$ and $|G|=2^{7}$. We have obtained the group stated in part (d1) of our proposition.

Case (2). Suppose that $|K: L|=2$ and $k \in \mathrm{Z}(G)$. Here we have $o(k)=4$ and $k^{2} \in\{u, u z\}$. If $k^{2}=u z$, then $(g t)^{2}=u z$ together with $[k, g t]=1$ implies that $g t k$ is an involution in $G-K$, a contradiction. Hence in this case we have $k^{2}=u$ and we have obtained the group of order $2^{6}$ stated in part $(d 2)$ of our proposition.

Case (3). Assume that $|K: L|=2$ and $k \notin \mathrm{Z}(G)$. We have

$$
k^{2}=u z^{\epsilon}, \epsilon \in\{0,1\},[k, t]=z^{\eta},[k, h]=z^{\delta}, \eta, \delta \in\{0,1\},
$$

and $\eta=\delta=0$ is not possible.

Then the fact that there are no involutions in $G-K$ gives a unique solution

$$
\epsilon=1, \eta=1, \delta=0
$$

and so we have obtained the special group of order $2^{6}$ stated in part (d3) of our proposition.

Conversely, all groups from part (d) of our proposition satisfy the condition $(*)$. 
(iii2) Suppose that $\langle h\rangle \leq \mathrm{Z}\left(G_{0}\right)$. We have for each $g \in G_{0}-L, G_{0}^{\prime}=\langle[g, t]\rangle$ with $[g, t]=u \in U-\langle z\rangle$ and $\langle z\rangle=\Omega_{1}(\langle h\rangle)$. We have

$$
\mathrm{Z}\left(G_{0}\right)=\langle h\rangle \times\langle u\rangle \cong \mathrm{C}_{p^{s}} \times \mathrm{C}_{p}, s \geq 2 .
$$

Since $1 \neq g^{p} \in \mathrm{Z}\left(G_{0}\right)$ and there are no elements of order $p$ in $G-K$, it follows that $A=\mathrm{Z}\left(G_{0}\right)\langle g\rangle$ is abelian of rank 2. Hence $A$ is either of type $\left(p^{s}, p^{2}\right)$ or $\left(p^{s+1}, p\right)$.

Suppose, by way of contradiction, that $A$ is of type $\left(p^{s}, p^{2}\right)$. In that case there is an element $g_{0} \in A-\mathrm{Z}\left(G_{0}\right)$ such that $g_{0}^{2}=u$, where $\langle u\rangle=G_{0}^{\prime}$. If $p=2$, then $\left\langle g_{0}, t\right\rangle \cong \mathrm{D}_{8}$ and so $g_{0} t$ is an involution in $G_{0}-K$, a contradiction. Hence we must have $p>2$ and $M=\left\langle g_{0}, t\right\rangle \cong \mathrm{M}_{p^{3}}$. By our assumptions, we have $M \unlhd G$. Note that $G^{\prime} \cap M=U \cap M=\langle u\rangle$ and set $C=\mathrm{C}_{G}(M)$ so that $C \cap M=\langle u\rangle$. If $C * M<G$, then $G / C \cong \mathrm{S}\left(p^{3}\right)$ (which is an $\mathrm{S}_{p}$-subgroup of Aut $(M))$, contrary to $U=G^{\prime} \leq C$. Hence we have $G=M * C$. Since $\langle h\rangle \leq C,\langle h\rangle \unlhd G$ and $t$ centralizes $C$, we have $C \leq K$ and so $K=C \times\langle t\rangle$. Because $C<K$ and $K^{\prime} \leq\langle z\rangle$, we have $C^{\prime} \leq\langle z\rangle$. If $C^{\prime}=\{1\}$, then $G^{\prime}=C^{\prime} M^{\prime}=\langle u\rangle$, a contradiction. Hence we have $C^{\prime}=\langle z\rangle$. Note that $\{1\} \neq K / L$ is cyclic, where $L=(\langle h\rangle U) \times\langle t\rangle$ and $K=C L$ with $C \cap L=\langle h\rangle U$. Thus $\{1\} \neq C /(\langle h\rangle \times\langle u\rangle)$ is cyclic. Let $c \in C$ be such that $\langle c\rangle$ covers $C /(\langle h\rangle \times\langle u\rangle)$ and so we must have $\langle[h, c]\rangle=\langle z\rangle$. Since $K / H$ is cyclic of order $\geq p^{2},\langle c\rangle$ also covers $K / H$ and so $\langle c\rangle$ covers $C /(H \cap C)=C /\langle h\rangle$. It follows that $C$ is metacyclic minimal nonabelian without a cyclic subgroup of index $p$ (noting that $\left.\mathrm{E}_{p^{2}} \cong \Omega_{1}(C)=U \leq \mathrm{Z}(G)\right)$. Hence we may set

$$
C=\langle a\rangle\langle b\rangle \text { with }\langle a\rangle>\langle z\rangle=C^{\prime},\langle a\rangle \cap\langle b\rangle=\{1\},\langle b\rangle \cong \mathrm{C}_{p^{r}}, r \geq 2,
$$

and $\Omega_{1}(\langle b\rangle)=\left\langle u z^{i}\right\rangle$, where $i$ is an integer $\bmod p$. Consider the subgroup

$$
\langle b\rangle \times\langle t\rangle \cong \mathrm{C}_{p^{r}} \times \mathrm{C}_{p},
$$

which is non-normal in $G$ since $\langle[a, b]\rangle=\langle z\rangle$ and $z \notin\langle b, t\rangle$. We claim that $\langle b, t\rangle$ is a maximal non-normal subgroup in $G$. Indeed, let $X>\langle b, t\rangle$ be a maximal non-normal subgroup in $G$. If $X \cap C>\langle b\rangle$, then $\langle z\rangle \leq X$ and so $\left\langle z, u z^{i}\right\rangle=G^{\prime} \leq X$, a contradiction. Hence we have $X \cap C=\langle\bar{b}\rangle$. Because $G / C \cong \mathrm{E}_{p^{2}}$, it follows that $X$ must contain an element $x \in G-(C \times\langle t\rangle)=$ $G-K$. On the other hand, $\mathrm{C}_{G}(t)=C \times\langle t\rangle=K$ and so $[x, t] \neq 1$ and $X$ is nonabelian, contrary to our assumptions. Finally, by Proposition 6 , we have $\left\langle b t^{j}\right\rangle \unlhd G$ for some integer $j \bmod p$, where $\Omega_{1}\left(\left\langle b t^{j}\right\rangle\right)=\left\langle u z^{i}\right\rangle$. On the other hand, we have

$$
\left[a, b t^{j}\right]=[a, b], \text { where }\langle[a, b]\rangle=\langle z\rangle \neq\left\langle u z^{i}\right\rangle,
$$

a final contradiction.

We have proved that $A=\mathrm{Z}\left(G_{0}\right)\langle g\rangle$ is abelian of type $\left(p^{s+1}, p\right)$. It follows that all elements of order $p^{s}$ in $\langle h\rangle U$ are central in $G$ (noting that $U \leq \mathrm{Z}(G)$ ). Replacing $H$ with $H^{*}=\langle t\rangle \times\left\langle h u^{i}\right\rangle$ for some integer $i \bmod p$ (which is also a maximal non-normal abelian subgroup of type $\left.\left(p^{s}, p\right)\right)$ so that $\left\langle g^{p}\right\rangle=\left\langle h u^{i}\right\rangle$ 
and then working with $H^{*}$ instead of $H$, we see that we may assume from the start that there is $g \in G-K$ such that $g^{p}=h$, where $\langle h\rangle \leq \mathrm{Z}(G)$ and we set $g^{p^{s}}=z$. If $t \in \mathrm{Z}(K)$, then $L \leq \mathrm{Z}(K)$ and since $K / L$ is cyclic, $K$ would be in that case abelian.

(iii2a) First assume that $K$ is nonabelian, i.e., $t \notin \mathrm{Z}(K)$. Then we have $K^{\prime}=\langle z\rangle$ and so if $k \in K-L$ is such that $\langle k\rangle$ covers $K / H$ (which is cyclic of order $\geq p^{2}$ ), then we may set (by choosing a suitable generator $t$ of $\langle t\rangle$ ) $[k, t]=z$.

It is easy to see that $\langle k\rangle$ splits over $H$. Indeed, if $\langle k\rangle$ does not split over $H$, then $\langle k\rangle \cap H=\langle k\rangle \cap\langle h\rangle$ since $\mathrm{Z}(G) \cap L=\langle h\rangle U$ and so we have $\langle k\rangle>\langle z\rangle$. It follows that $\langle k, t\rangle \cong \mathrm{M}_{p^{n+1}}$ with $n \geq 3$ since $[k, t]=z$. On the other hand, $[g, t] \in U-\langle z\rangle$ and so $[g, t] \notin\langle k, t\rangle$ which implies that $\langle k, t\rangle$ is not normal in $G$, contrary to our assumptions. Hence $\langle k\rangle$ splits over $H$ and we may set $o(k)=p^{r}, r \geq 2$, and $k^{p^{r-1}}=u \in U-\langle z\rangle$.

If $o\left(k^{p}\right)>p^{s}$, then $\langle t\rangle \times\left\langle k^{p}\right\rangle \cong \mathrm{C}_{p} \times \mathrm{C}_{p^{r-1}}$ is non-normal in $G$ (since we have $\left.[k, t]=z \notin\left\langle t, k^{p}\right\rangle\right)$, contrary to the maximality of $|H|=p^{s+1}$. Hence we have $r \leq s+1$. We set $[g, t]=u^{i} z^{j}$ with $i \not \equiv 0(\bmod p)$.

We have here

$$
\Phi(G)=\mho_{1}(G)=\mathrm{Z}(G)=\left\langle g^{p}\right\rangle \times\left\langle k^{p}\right\rangle \text { and so }|G: \Phi(G)|=p^{3} .
$$

By Lemma 146.7 in [4], $G$ possesses a unique abelian maximal subgroup $A^{*}$. Because we have $\left|G: \mathrm{C}_{G}(t)\right|=p^{2}$, it follows that $t \in G-A^{*}$ and

$$
\mathrm{C}_{A^{*}}(t)=\mathrm{Z}(G)=\langle h\rangle \times\left\langle k^{p}\right\rangle, A^{*} / \mathrm{Z}(G) \cong G^{\prime}=U=\Omega_{1}\left(A^{*}\right)
$$

so that $A^{*}$ is of rank 2 and of type $\left(p^{s+1}, p^{r}\right)$, where $s \geq 2$ and $2 \leq r \leq s+1$. Indeed, the map $a \rightarrow[a, t]\left(a \in A^{*}\right)$ is a homomorphism from $A^{*}$ onto $G^{\prime}$ and so $A^{*} / \mathrm{Z}(G) \cong G^{\prime}$.

Case (a): $r<s+1$. In this case we may set

$$
A^{*}=\langle a\rangle \times\langle b\rangle, \text { where }\langle a\rangle \cong \mathrm{C}_{p^{s+1}},\langle b\rangle \cong \mathrm{C}_{p^{r}}, z=a^{p^{s}}, u=b^{p^{r-1}} .
$$

Take an element $a^{\prime} \in\left\langle a^{p}\right\rangle \leq \mathrm{Z}(G)$ such that $o\left(a^{\prime}\right)=p^{r}$ and $\left(a^{\prime}\right)^{p^{r-1}}=z$. Suppose that $[b, t] \notin\langle z\rangle$. Then we have $[b, t]=z^{i} u(i$ is an integer $\bmod p)$ for a suitable choice of a generator $t$ of $\langle t\rangle$. We get

$$
\left(\left(a^{\prime}\right)^{i} b\right)^{p^{r-1}}=z^{i} u \text { and }\left[\left(a^{\prime}\right)^{i} b, t\right]=[b, t]=z^{i} u
$$

and therefore we have either $p=2, r=2$ and $\left\langle\left(a^{\prime}\right)^{i} b, t\right\rangle \cong \mathrm{D}_{8}$ or $\left\langle\left(a^{\prime}\right)^{i} b, t\right\rangle \cong$ $\mathrm{M}_{p^{r+1}}$ (where in case $p=2$, we have $r \geq 3$ ). But $\left|G: \mathrm{C}_{G}(t)\right|=p^{2}$ and so for some $g \in G$ we get $\langle[g, t]\rangle \neq\left\langle z^{i} u\right\rangle$ and so $\left\langle\left(a^{\prime}\right)^{i} b, t\right\rangle$ is not normal in $G$, contrary to our assumptions. Hence choosing a suitable generator $t$ of $\langle t\rangle$, we must have $[b, t]=z$. Then we also get $[a, t]=u^{i} z^{j}$ with $i \neq \equiv 0(\bmod p)$.

Case (b): $r=s+1$. Let $b \in A^{*}-\Phi(G)$ be such that $[b, t]=z$ and set $b^{p^{s}}=u$, where $\langle u\rangle \neq\langle z\rangle$. Let $a \in A^{*}-\Phi(G)$ be such that $a^{p^{s}}=z$ and then 
we have

$$
A^{*}=\langle a\rangle \times\langle b\rangle \cong \mathrm{C}_{p^{s+1}} \times \mathrm{C}_{p^{s+1}} \text { and }[a, t]=u^{i} z^{j}, i \not \equiv 0(\bmod p) .
$$

In this critical case we must also have $j \not \equiv \xi-i \xi^{-1}(\bmod p)$ for all integers $\xi \not \equiv 0 \quad(\bmod p)$. Indeed, assume that for some $\xi \not \equiv 0(\bmod p)$, we have $j \equiv \xi-i \xi^{-1}(\bmod p)$. In that case we solve the congruence $i \mu \equiv \xi(\bmod$ $p$ ) with some $\mu \not \equiv 0(\bmod p)$. We compute (noting that $s \geq 2)$ :

$$
\left(a^{\mu} b\right)^{p^{s}}=\left(a^{p^{s}}\right)^{\mu} b^{p^{s}}\left[b, a^{\mu}\right]^{\left(\begin{array}{c}
p^{s} \\
2
\end{array}\right)}=z^{\mu} u
$$

and

$$
\begin{gathered}
{\left[a^{\mu} b, t\right]=\left(u^{i} z^{j}\right)^{\mu} z=z^{1+j \mu} u^{i \mu}=z^{1+\left(\xi-i \xi^{-1}\right) \mu} u^{\xi}=} \\
z^{1+\xi \mu-\xi^{-1} i \mu} u^{\xi}=z^{1+\xi \mu-1} u^{\xi}=z^{\xi \mu} u^{\xi}=\left(z^{\mu} u\right)^{\xi} .
\end{gathered}
$$

It follows that $\left\langle a^{\mu} b, t\right\rangle \cong \mathrm{M}_{p^{s+2}}, s \geq 2$, and since $[b, t]=z \notin\left\langle a^{\mu} b, t\right\rangle$, it follows that $\left\langle a^{\mu} b, t\right\rangle$ is not normal in $G$, contrary to our assumptions. We have obtained the groups stated in part (e) of our proposition.

Conversely, we see that in any group $G$ from part (e) of our proposition, for each $x \in A^{*}-\mathrm{Z}(G),\langle x\rangle$ is not normal in $G$ and so $\mathrm{D}_{8}$ or $\mathrm{M}_{p^{n}}$ cannot be subgroups of $G$, where $A^{*}$ is the unique abelian maximal subgroup of $G$. Furthermore, let $X$ be any maximal non-normal abelian subgroup of $G$ of order $\geq p^{3}$ which has more than one subgroup of order $p$. Since $G$ has exactly one conjugacy class of noncentral subgroups of order $p$ with the representative $\langle t\rangle$, we may assume that $t \in X$. It follows that $X=\langle t\rangle \times X_{0}$, where $X_{0}$ is any maximal cyclic subgroup in $\mathrm{Z}(G)$. Hence our condition $(*)$ holds.

(iii2b) It remains to consider the case $t \in \mathrm{Z}(K)$ so that $K$ is abelian and $K>L$. Since $K / \mathrm{C}_{K}(g) \cong G^{\prime}$ (Lemma 1.1 in [1]), there is $k \in K-L$ such that $\langle k\rangle$ covers $K / H$ and $[g, k]=z=g^{p^{s}}, s \geq 2$, where $[g, t] \in U-\langle z\rangle$ with $p^{r}=o(k) \geq p^{2}$. Since $K=\langle t\rangle \times\langle h, k\rangle$ and $\mathrm{Z}(G)=\left\langle h, k^{p}\right\rangle$, it follows that $U \leq\left\langle h, k^{p}\right\rangle$ because $U \leq \mathrm{Z}(G)$. Hence we have $\Omega_{1}(\langle h, k\rangle)=U=G^{\prime}$. Consider the subgroup

$$
\langle t\rangle \times\langle k\rangle \cong \mathrm{C}_{p} \times \mathrm{C}_{p^{r}}, r \geq 2 .
$$

If $\Omega_{1}(\langle k\rangle)=\langle z\rangle$, then $[g, t] \in U-\langle z\rangle$ shows that $\langle t, k\rangle$ is not normal in $G$. If we have $\Omega_{1}(\langle k\rangle)=\langle u\rangle$ with $u \in U-\langle z\rangle$, then $[g, k]=z$ shows that again $\langle t, k\rangle$ is not normal in $G$. The maximality of $|H|$ shows that we must have $r \leq s$ and so we have $\exp (K)=p^{s}$. It follows that $\langle h\rangle$ splits in $\langle h, k\rangle$ and so we have

$$
\langle h, k\rangle=\langle h\rangle \times\left\langle k^{\prime}\right\rangle \text { with } \Omega_{1}\left(\left\langle k^{\prime}\right\rangle\right)=\langle u\rangle, u \in U-\langle z\rangle \text { and } o\left(k^{\prime}\right) \geq p^{2} .
$$

Since $[g, t] \in U-\langle z\rangle$, there is an integer $j \bmod p$ so that $\left[g, t^{j} k^{\prime}\right]=z$. Because $\Omega_{1}\left(\left\langle t^{j} k^{\prime}\right\rangle\right)=\langle u\rangle$, we may assume from the start that (replacing $k$ with $t^{j} k^{\prime}$ 
and writing $k$ again):

$$
\begin{aligned}
& K=\langle t\rangle \times\langle h\rangle \times\langle k\rangle, o(k)=p^{r}, 2 \leq r \leq s, \\
& k^{p^{r-1}}=u \in U-\langle z\rangle \text { and }[g, k]=z=g^{p^{s}} .
\end{aligned}
$$

Replacing $t$ with some other generator of $\langle t\rangle$ (if necessary), we may assume from the start that $[g, t]=u z^{i}$ for some integer $i \bmod p$.

For any integer $\alpha \not \equiv 0(\bmod p)$ and any $x \in K$, we have (noting that $s \geq 2$ )

$$
\left(g^{\alpha} x\right)^{p^{s}}=z^{\alpha}\left[x, g^{\alpha}\right]^{\left(\begin{array}{c}
p^{s} \\
2
\end{array}\right)}=z^{\alpha}
$$

and so $\Omega_{1}(G)=\langle t\rangle \times U \cong \mathrm{E}_{p^{3}}$ and the socle of each cyclic subgroup of $G$ which is not contained in $K$ is equal $\langle z\rangle$.

Let $h^{\prime}$ be an element of order $p^{r}$ in $\langle h\rangle$ such that $\left(h^{\prime}\right)^{p^{r-1}}=z$. For any fixed $\alpha \not \equiv 0(\bmod p)$ we consider the subgroup

$$
\langle t\rangle \times\left\langle\left(h^{\prime}\right)^{\alpha} k\right\rangle \cong \mathrm{C}_{p} \times \mathrm{C}_{p^{r}}, \text { where }\left(\left(h^{\prime}\right)^{\alpha} k\right)^{p^{r-1}}=z^{\alpha} u
$$

and note that $\left\langle\left(h^{\prime}\right)^{\alpha} k\right\rangle \cong \mathrm{C}_{p^{r}}, r \geq 2$, is a maximal cyclic subgroup in $G$ with the socle $\left\langle z^{\alpha} u\right\rangle$. We have $\left[g,\left(h^{\prime}\right)^{\alpha} k\right]=z \notin\left\langle t,\left(h^{\prime}\right)^{\alpha} k\right\rangle$ so that $\left\langle t,\left(h^{\prime}\right)^{\alpha} k\right\rangle$ is a maximal non-normal subgroup in $G$. By Proposition 6 , there is a unique integer $j(\bmod p)$ such that $\left\langle t^{j}\left(h^{\prime}\right)^{\alpha} k\right\rangle \unlhd G$. Hence we must have:

$$
\left[g, t^{j}\left(h^{\prime}\right)^{\alpha} k\right]=\left(u z^{i}\right)^{j} z=z^{1+i j} u^{j} \in\left\langle z^{\alpha} u\right\rangle
$$

which shows that $j \not \equiv 0(\bmod p)$ and we get

$$
z^{1+i j} u^{j}=z^{\alpha j} u^{j} \text { so that } 1+i j \equiv \alpha j \text { or } j(\alpha-i) \equiv 1(\bmod p) .
$$

Hence for any fixed $\alpha \not \equiv 0(\bmod p)$, there must exist $j \not \equiv 0(\bmod p)$ such that $j(\alpha-i) \equiv 1(\bmod p)$ and this gives that we must have $i \equiv 0(\bmod p)$. We have obtained the relation $[g, t]=u$.

Because $[g, k]=z$ and $\langle k\rangle \cong \mathrm{C}_{p^{r}}, r \geq 2$, is a maximal cyclic subgroup in $G$ with the socle $\langle u\rangle$, it follows that $\langle t\rangle \times\langle k\rangle \cong \mathrm{C}_{p} \times \mathrm{C}_{p^{r}}$ is a maximal non-normal subgroup in $G$. By Proposition 6 , there is a unique integer $m$ ( $\bmod p)$ such that $\left\langle t^{m} k\right\rangle \unlhd G$. But we have

$$
\left[g, t^{m} k\right]=[g, t]^{m}[g, k]=u^{m} z,
$$

a final contradiction (since $\Omega_{1}\left(\left\langle t^{m} k\right\rangle\right)=\langle u\rangle$ ). Our proposition is completely proved.

Proof of Theorem C. By inspection of all Propositions 1 to 12, we see that all possible cases have been investigated and so our theorem is proved. 


\section{REFERENCES}

[1] Y. Berkovich, Groups of prime power order, Vol. 1, Walter de Gruyter, Berlin-New York, 2008.

[2] Y. Berkovich and Z. Janko, Groups of prime power order, Vol. 2, Walter de Gruyter, Berlin-New York, 2008.

[3] Y. Berkovich and Z. Janko, Groups of prime power order, Vol. 3, Walter de Gruyter, Berlin-New York, 2011.

[4] Y. Berkovich and Z. Janko, Groups of prime power order, Vol. 4, Walter de Gruyter, Berlin-New York, to appear 2014.

Z. Janko

Mathematical Institute

University of Heidelberg

69120 Heidelberg

Germany

E-mail: janko@mathi.uni-heidelberg.de

Received: 1.3.2014. 